\title{
NBSIR 81-2345
}

\section{A Report on Some Thermodynamic Data for Desulfurization Processes}

U.S. DEPARTMENT OF COMMERCE National Bureau of Standards Center for Chemical Physics Chemical Thermodynamics Division Washington, DC 20234

September 1981

Final

\section{Prepared for}

Department of Energy

Morgantown Energy Technology Center

Morgantown, West Virginia

QC

100 of Standard Reference Data, NBS

.456

\#81.2345 
NBSIR $81-2345$

\section{A REPORT ON SOME THERMODYNAMIC DATA FOR DESULFURIZATION PROCESSES}

V. B. Parker, B. R. Staples, T. L. Jobe, Jr., and D. B. Neumann

U.S. DEPARTMENT OF COMMERCE

National Bureau of Standards

Center for Chemical Physics

Chemical Thermodynamics Division

Washington, DC 20234

September 1981

Final

Prepared for

Department of Energy

Morgantown Energy Technology Center

Morgantown, West Virginia

and

Office of Standard Reference Data, NBS

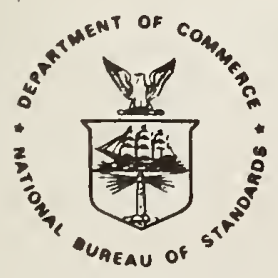

U.S. DEPARTMENT OF COMMERCE, Malcolm Baldrige, Secretary NATIONAL BUREAU OF STANDARDS, Ernest Ambler, Director 


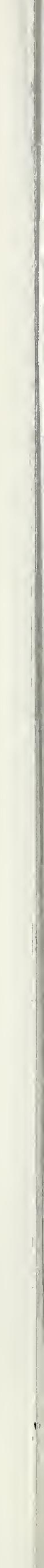




\section{TABLE OF CONTENTS}

I. General Description

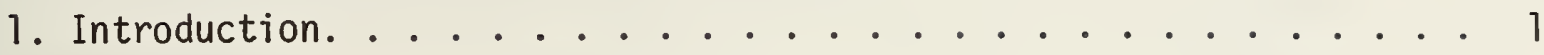

2. Explanation of the Contents of the Tables ......... 3

3. Unit of Energy and Fundamental Constants . . . . . . . 8

4. Internal Consistency of the Tables . . . . . . . . 8

II. Tables for the Thermochemical Property Values of Substances.

1. The $0, \mathrm{H}, \mathrm{S}$, or $\mathrm{C}$ containing substances ........ 16

2. The $\mathrm{Fe}-\mathrm{O}, \mathrm{H}, \mathrm{S}$, or $\mathrm{C}$ containing substances........ 18

3. The $\mathrm{Mn}-\mathrm{O}, \mathrm{H}, \mathrm{S}$, or $\mathrm{C}$ containing substances........ 20

4. The $\mathrm{Mg}-\mathrm{O}, \mathrm{H}, \mathrm{S}$, or $\mathrm{C}$ contairing substances ........ 21

5. The $\mathrm{Ca}-\mathrm{O}, \mathrm{H}, \mathrm{S}$, or $\mathrm{C}$ containing substances........ 23

6. The $\mathrm{Na}-\mathrm{O}, \mathrm{H}, \mathrm{S}$, or $\mathrm{C}$ containing substances......... 24

7. The $\mathrm{K}-\mathrm{O}, \mathrm{H}, \mathrm{S}$, or $\mathrm{C}$ containing substances ........ 26

III. Tables for the Thermochemical Values for Processes

1. Processes involving $0, \mathrm{H}, \mathrm{S}$, or $\mathrm{C}$ containing substances. . . 28

2. Processes involving $\mathrm{Fe}-\mathrm{O}, \mathrm{H}, \mathrm{S}$, or $\mathrm{C}$ containing substances. . 30

3. Processes involving $M n-0, H, S$, or $C$ containing substances. . 32

4. Processes involving $\mathrm{Mg}-\mathrm{O}, \mathrm{H}, \mathrm{S}$, or $\mathrm{C}$ eontaining substances. . 33

5. Processes involving $\mathrm{Ca}-\mathrm{O}, \mathrm{H}, \mathrm{S}$, or $\mathrm{C}$ containing substances. . 35

6. Processes involving $\mathrm{Na}-\mathrm{O}, \mathrm{H}, \mathrm{S}$, or $\mathrm{C}$ containing substances. . 36

7. Processes involving $\mathrm{K}-\mathrm{O}, \mathrm{H}, \mathrm{S}$, or $\mathrm{C}$ containing substances... 38 
IV. Tables for the Property Values of Binary Aqueous Systems as a Function of Concentration

1. $\mathrm{SO}_{2} \ldots \ldots \ldots \ldots . \ldots \ldots 4 . \ldots \ldots$

2. $\mathrm{H}_{2} \mathrm{SO}_{3} \ldots \ldots \ldots . \ldots . \ldots . \ldots . \ldots . \ldots 4$

3. $\mathrm{H}_{2} \mathrm{SO}_{4} \ldots \ldots \ldots \ldots . \ldots . \ldots . \ldots . \ldots 4$

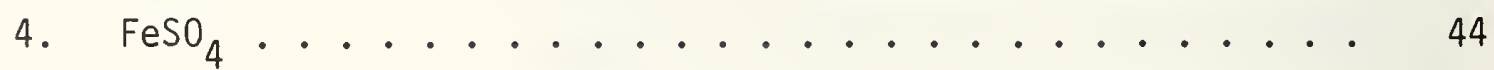

5. $\mathrm{Fe}_{2}\left(\mathrm{SO}_{4}\right)_{3} \ldots \ldots \ldots \ldots . \ldots . \ldots . \ldots 45$

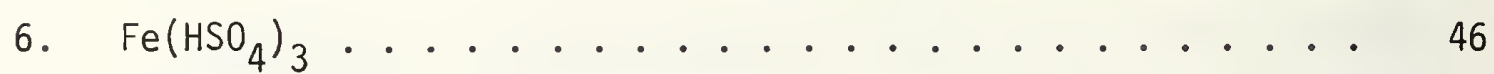

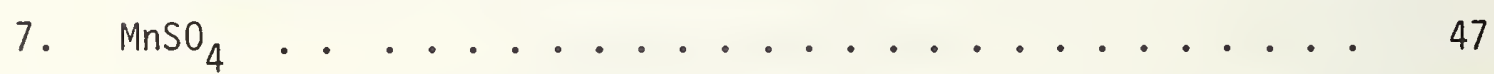

8. $\mathrm{MgSO}_{4} \ldots \ldots \ldots \ldots . \ldots . \ldots . \ldots 48$

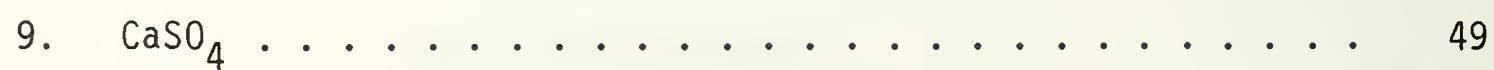

10. $\mathrm{CaS}_{2} \mathrm{O}_{3} \ldots \ldots \ldots \ldots \ldots \ldots$

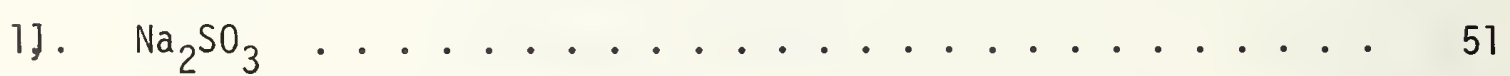

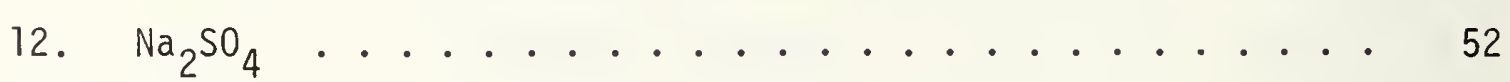

13. $\mathrm{NaHSO}_{4}$.......................... 53

14. $\mathrm{KOH} \ldots \ldots \ldots . \ldots . \ldots . . . \ldots 54$

15. $\mathrm{K}_{2} \mathrm{SO}_{3} \ldots \ldots \ldots \ldots \ldots$

16. $\mathrm{K}_{2} \mathrm{SO}_{4} \ldots \ldots \ldots \ldots 56$

17. $\mathrm{KHSO}_{3} \ldots \ldots \ldots \ldots 5$

18. $\mathrm{KHSO}_{4} \ldots \ldots \ldots \ldots \ldots$

V. Documentation for the Thermochemical Values for Some Processes

1. Processes involving $\mathrm{Fe} \ldots . . . . . . . .660$

2. Processes involving $\mathrm{Mg} \ldots \ldots 1 . \ldots . . \ldots 61$

3. Processes involving $\mathrm{Ca}$.................. 65

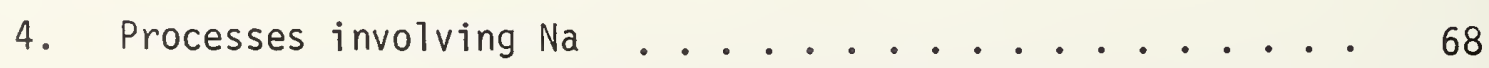


VI. Documentation and Discussion of the Aqueous Solution Property Values

1. Activity and Osmotic Coefficients . . . . . . . . . . . 71

2. The Relative Apparent Molar Enthalpy . . . . . . . . . . . 74

VII. Bibliography 76 


\section{A REPORT ON SOME THERMODYNAMIC \\ DATA FOR DESULFURIZATION PROCESSES}

by

V. B. Parker, B.R. Staples, T.L. Jobe, and D. Neumann

Abstract

Tables are presented here of values of thermochemical properties and processes at $298.15 \mathrm{~K}$ for some substances of interest to DOE for flue gas desulfurization.

The substances covered are (1) the aqueous ions: $\mathrm{OH}^{-}, \mathrm{SO}_{3}^{-2}, \mathrm{HSO}_{3}^{-}$, $\mathrm{SO}_{4}^{-2}, \mathrm{HSO}_{4}^{-}, \mathrm{CO}_{3}^{-2}, \mathrm{HCO}_{3}^{-}, \mathrm{H}^{+}, \mathrm{Mn}^{+2}, \mathrm{Fe}^{+2}, \mathrm{Mg}^{+2}, \mathrm{Ca}^{+2}, \mathrm{Na}^{+}$, and $\mathrm{K}^{+}$, and (2) solid, liquid, aqueous, and gaseous compounds or species formed from these ions.

The tables contain the following:

1. The thermochemical property values, enthalpy of formation, $\Delta_{f} H^{\circ}$, Gibbs energy of formation, $\Delta_{f} G^{\circ}$, entropy, $S^{\circ}$, and heat capacity, $C_{p}^{\circ}$,all at 298.15 K, as well as the enthalpy difference between $298.15 \mathrm{~K}$ and $0 \mathrm{~K}$, $H^{\circ}-H_{0}^{\circ}$, for the basic species cited above.

2. The predicted values for $\Delta H^{\circ}, \Delta G^{\circ}, \Delta S^{\circ}$, and $\Delta C_{p}^{\circ}$ as well as $\log K$ (equilibrium constant) for the processes, or reactions, of importance to DOE, calculated from (1).

3. The property values, $\phi_{L}$, the relative apparent molar enthalpy, $\gamma_{ \pm}$, the mean ionic activity coefficient, and $\phi$, the osmotic coefficient, for the binary aqueous systems at $298.15 \mathrm{~K}$, all as a function of concentration.

Some documentation for (2) and (3) is provided. All of the values given are consistent with the NBS TN 270 Series. 


\section{GENERAL DESCRIPTION}

\section{Introduction}

In this report tables of values of thermochemical properties and processes at $298.15 \mathrm{~K}$ for substances of interest to DOE for flue gas desulfurization processes are presented. This work is part of an ongoing evaluation of the various systems of interest. An analysis of the more recent data is underway and these data will be incorporated into these tables in a way which will preserve the total unity and consistency of the tables.

The substances covered are: (1) the aqueous ions: $\mathrm{OH}^{-}, \mathrm{SO}_{3}^{-2}, \mathrm{HSO}_{3}^{-}$, $\mathrm{SO}_{4}^{-2}, \mathrm{HSO}_{4}^{-}, \mathrm{CO}_{3}^{-2}, \mathrm{HCO}_{3}^{-}, \mathrm{H}^{+}, \mathrm{Mn}^{+2}, \mathrm{Fe}^{+2}, \mathrm{Mg}^{+2}, \mathrm{Ca}^{+2}, \mathrm{Na}^{+}$, and $\mathrm{K}^{+}$, and (2) solid, liquid, aqueous, and gaseous compounds or species formed from these ions.

The tables are arranged in three sections.

Section II contains the thermochemical property values, $\Delta_{f} H^{\circ}, \Delta_{f} G^{\circ}$, and $\left(\mathrm{H}-\mathrm{H}_{0}^{\circ}\right.$ ) in $\mathrm{kcal} / \mathrm{mol}$, and $\mathrm{S}^{\circ}$ and $\mathrm{C}_{\mathrm{p}}^{\circ}$ in $\mathrm{cal} /(\mathrm{mol} \cdot \mathrm{K})$, all at $298.15 \mathrm{~K}$, for the basic substances of interest. They are either obtained from the NBS Technical Note 270 Series, "Selected Values of Chemical Thermodynamic Properties of the Elements and Their Compounds, "[81NBS] $]^{1}$, or have been selected to be compatible with those tables.

1 References are given in a chronologically ordered bibliography at the end of the report (section VII). They are cited in the text in coded form indicating year of publication and the first two authors. The pre 1900 references are listed before and separately from the post 1900 ones. 
Section III contains the values, $\Delta H^{\circ}, \Delta G^{\circ}$ in $\mathrm{kcal} / \mathrm{mol}, \Delta S^{\circ}$ and $\Delta C_{p}^{\circ}$ in $\mathrm{cal} /(\mathrm{mol} \cdot \mathrm{K})$, and $\log \mathrm{K}$ for some of the important processes in flue gas desulfurization. Emphasis has been given to the values for solution processes, i.e., $\Delta H^{\circ}(\operatorname{soln}), \Delta G^{\circ}(\operatorname{soln}), \Delta S^{\circ}(\operatorname{soln}), \log K_{s}^{\circ}$ and $\Delta C_{p}^{\circ}$ for the salts and their hydrates at $298.15 \mathrm{~K}$, as well as to the $\mathrm{K}$ 's and $\Delta H^{\prime} \mathrm{s}$ for ion pairing or complex ion formation. The process values are derived from the property values listed in section II as, for example:

$\Delta H^{\circ}=\Sigma \Delta_{f} H^{\circ}$ (products) $-\Sigma \Delta_{f} H^{\circ}$ (reactants).

Section IV contains the following property values of various solutions as a function of concentration: $\Delta_{f} H$, and the derived $\phi_{L}$, (the relative apparent molar enthalpy) and $\gamma_{ \pm}$(mean ionic activity coefficient) and $\phi$ (the osmotic coefficient).

Documentation for section III is provided in section $V$, as is documentation for section IV in section VI. Detailed analyses and complete documentation, however, are not given at this time since it is beyond the scope of this report. Many of the selections for the properties of the substances involve analysis of a complex network of thermodynamic data. In that process values for individual processes or substances may become modified in order to achieve a set of "best values." Some documentation, however, is provided on some processes where the experimental measurements on a particular process are the definitive ones for the predicted properties of that process or where newer data indicate the need for revision. Documentation is also provided for the activity coefficients given in the tables on solution properties.

Users of these tables are invited to comment on the selections, correct errors and bring new measurements to our attention. The support of the Department. of Energy and Office of Standard Reference Data, NBS is gratefully acknowledged. 


\section{Explanation of the contents of the tables}

The following material, adapted from [81NBS] provides definitions and conventions used in the tables.

\subsection{Physical States}

The physical state of the substance is appended to the chemical formula in parentheses: $\mathrm{NaOH}(\mathrm{c})=\mathrm{NaOH}$, crystalline. The abbreviations most commonly used to denote physical states are listed in Table I-1. Any other notations are explained in the tables.

\subsection{Definition of Symbols for the Thermochemical Properties} and Thermochemical Processes

The headings used in the tables in section II and their meanings are:

$\Delta_{\mathrm{f}} \mathrm{H}^{\circ} \quad$ standard enthalpy of formation at $298.15 \mathrm{~K}$;

$\Delta_{f} G^{\circ} \quad$ standard Gibbs energy, $G$, of formation at $298.15 \mathrm{~K}$;

$H^{\circ}-\mathrm{ri}_{\mathrm{O}}^{\circ}$ enthalpy, $\mathrm{H}$, of the compound in the indicated state at $298.15 \mathrm{~K}$ referred to its value at $0 \mathrm{~K}$. If the indicated state at $298 \mathrm{~K}$ is gas, the corresponding state at $0 \mathrm{~K}$ is the hypothetical ideal gas; if the state at $298 \mathrm{~K}$ is solid or liquid, the corresponding state at $0 \mathrm{~K}$ is the thermodynamically stable crystalline solid, unless otherwise specifically indicated.

$S^{\circ} \quad$ standard entropy, $S$, at $298.15 \mathrm{~K}$;

$\mathrm{C}_{\mathrm{p}}^{\circ} \quad$ heat capacity at constant pressure at $298.15 \mathrm{~K}$.

The Gibbs energy is related to the other quantities by: $G=H-T S$, where $T$ is the thermodynamic temperature. The enthalpy is related to the internal energy, $E$, by: $H=E+P V$, where $P=$ pressure and $V=$ volume.

All values refer to one mole of substance for the formula given. 
For the processes listed in the tables in section III the headings and their meanings are:

$\Delta H^{\circ} \quad$ enthalpy change for the process under standard conditions, i.e., for the substances in their standard states, at $298.15 \mathrm{~K}$

$\Delta G^{\circ} \quad$ Gibbs energy change for the process under standard conditions, etc.

$\Delta S^{\circ} \quad$ Entropy change for the process under standard conditions, etc.

$\Delta \mathrm{C}_{\mathrm{p}}^{\circ} \quad$ heat capacity change at constant pressure at $298.15 \mathrm{~K}$

$\log \mathrm{K}$ The common logarithm of the equilibrium constant, equal to $-\Delta G^{\circ} / 2.3026 R T$

The tables in section IV for the solution properties have the following headings and meanings.

$\phi_{L} \quad$ the relative apparent molar enthalpy

$\gamma_{ \pm} \quad$ the mean ionic activity coefficient

$\phi \quad$ the osmotic coefficient.

\subsection{Conventions Regarding Pure Substances}

The values of the thermodynamic properties of the pure substances given in the tables in section II are for the substances in their standard states. These standard states are defined as follows:

For a pure solid or liquid, the standard state at any temperature is the substance in the condensed phase under a pressuré of one atmosphere.

For a gas the standard state at any temperature is the hypothetical ideal gas at unit fugacity, in which state the enthalpy is that of the real gas at the same temperature and at zero pressure.

The phase of a substance is indicated in parentheses at the end of the chemical formula. See section I-2.1. 
The values of $\Delta_{f} H^{\circ}$ and $\Delta_{f} G^{\circ}$ given in the tables represent the change in the appropriate thermodynamic quantity when one mole of the substance in its standard state is formed, isothermally at the indicated temperature, from the elements, each in its appropriate standard reference state. The standard reference state at $25^{\circ} \mathrm{C}$ for each element except phosphorus has been chosen to be the standard state that is thermodynamically stable at $25^{\circ} \mathrm{C}$ and at one atmosphere pressure. For phosphorus the standard reference state is the crystalline white form; the more stable forms have not been well characterized thermochemically. The same reference states have been maintained for the elements at $0 \mathrm{~K}$ except for the liquid elements bromine and mercury for which the reference states have been chosen as the stable crystalline forms. The standard reference states for the elements are indicated in the tables by the fact that the values of $\Delta_{f} H^{\circ}$ and $\Delta_{f} G^{\circ}$ are exactly zero.

The values of $S^{\circ}$ represent the virtual or "thermal" entropy of the substance in the standard state at $298.15 \mathrm{~K}$, omitting contributions from nuclear spins. Isotope mixing effects, etc., are also excluded except in the case of the hydrogen-deuterium $\left({ }^{1} \mathrm{H}_{-}{ }^{2} \mathrm{H}\right)$ system. Where data have been available only for a particular isotope, they have been corrected, when possible, to the normal isotopic composition.

\subsection{Convention Regarding Solutions}

For all dissolved substances the composition of the solvent is indicated in parentheses following the chemical formula. Except in special cases, discussed below, the number of moles of the solvent associated with one mole of solute is stated explicitly. See section I- 2.1 and Table I-1 for the conventions used. 
The standard state for a non-dissociated solute in aqueous solution is taken as the hypothetical ideal solution of unit molality, which has been designated as "std state, $m=1$." For strong electrolytes in aqueous solution the conventional standard state is the ideal solution of unit activity (unit mean molality). For non-aqueous solutions the standard state of the solute is the hypothetical ideal solution of unit mole fraction of solute (std. state, $x_{2}=1$ ). The designation "a" is used for strong electrolytes in the standard state and "ao" for undissociated species in water solution. In non-aqueous media the standard state defined above is indicated by appending " $X$ " to the formula of the solvent. The convention "std state, $m=1$ " is used only rarely for non-aqueous media. An "s" is appended to the formula of the solvent to designate this condition.

The value for $\Delta_{f} H$ for a solute in a solution of definite composition is the apparent enthalpy of the reaction of formation of the real solution from the elements comprising the solute, each in its standard reference state, and the appropriate total number of moles of solvent.

Elements + solvent $\rightarrow$ salt (dissolved in solvent). When using this representation for $\Delta_{f} H$ for the dissolved species the value of $\Delta_{f} H$ for the solvent is not required; the experimental value of an enthalpy of dilution can be obtained directly as the difference between the two values of $\Delta_{f} H$ at the corresponding concentrations.

It may be noted, to avoid confusion, that $\Delta_{f} H$ applies to the real solution, including any hydrolysis, ionization or other reaction that occurs upon solution of the solute. 
The value for $\Delta_{f} H^{\circ}$ for a solute in its standard state is equal to the apparent molar enthalpy of formation of the substance in an ideal solution containing the species of which the solute is assumed to be nominally composed. If this speciation is the same as that in the real solution at infinite dilution, then $\Delta_{f} H^{\circ}$ is equal to $\Delta_{f} H^{\infty}$, the enthalpy of dilution of an ideal solution being zero. The assumed nominal composition of the solute is that at infinite dilution for non-dissociating substances, salts composed of ions that do not react further, such as $\mathrm{NaI}$, and $\mathrm{KMnO}_{4}$, and the common acids, such as $\mathrm{HCl}, \mathrm{H}_{2} \mathrm{SO}_{4}, \mathrm{H}_{3} \mathrm{PO}_{4}$ and acetic acid. THe assumed nominal composition for salts containing ionizable or hydrolyzable species is not the same as the speciation in the real, infinitely dilute solution. For example, $\mathrm{NaHSO}_{4}$ is composed of $\mathrm{Na}^{+}$and $\mathrm{HSO}_{4}^{-}$ions in its standard state, while the infinitely dilute real solution contains $\mathrm{Na}^{+}, \mathrm{H}^{+}$and $\mathrm{SO}_{4}{ }^{2-}$ ions. THe difference between the $\Delta_{\mathrm{f}} \mathrm{H}^{\prime} \mathrm{s}$ for these two solutions is the enthalpy of ionization of $\mathrm{HSO}_{4}^{-}$.

The values of the thermodynamic properties tabulated for the individual ions in aqueous solution are based on the usual convention that the values of $\Delta_{f} H^{\circ}, \Delta_{f} G^{\circ}, S^{\circ}$ and $C_{p}^{\circ}$ for $H^{+}(a q$, std. state, $m=1)$ are zero. For an ionic species e.g., $\mathrm{HSO}_{4}^{-}$, the properties tabulated refer to that undissociated ion, i.e. they are not equal to the sum of those for its constituent ions. By adopting the above convention with respect to aqueous $\mathrm{H}^{+}$, it follows that the thermodynamic relation $\Delta_{f} G^{\circ}=\Delta_{f} H^{\circ} \quad-T\left[\Delta_{f} S^{\circ}+[n / 2] S^{\circ}\left(H_{2}(g: s)\right)\right]$ where $\Delta_{f} S^{\circ}$ has been calculated from tabulated entropies holds for individual ionic species, with $n$ equal to the algebraic value of the charge. For neutral electrolyes, (and gaseous ions) the normal consistency relationship applies. See section I-4. 


\section{Unit of Energy and Fundamental Constants}

A11 of the energy values given in these tables are expressed in terms of the thermochemical calorie. This unit, defined as equal to 4.184 joules exactly, is general7y accepted for the presentation of chemical thermodynamic data. Values reported in other units have been converted to calories by means of the conversion factors for molecular energy given in Table I-2.

The values in this table are consistent with the CODATA Fundamental Constants [73COD] and negligibly different from those listed in [81NBS].

The formula weights used in the tables are those listed in [87NBS]. Use of more recent atomic weights [80IUP] would produce only insignificant changes in the tabulated thermodynamic values.

\section{Internal Consistency of the Tables}

A11 of the values given in the tables of thermochemical properties have been calculated from the original articles, using consistent values for all subsidiary and auxiliary quantities. The original data were corrected where possible for differences in energy units, molecular weights, temperature scales, etc. Thus we have sought to maintain a uniform scale of energies for all substances in the tables. In addition the tabulated values of the properties of a substance satisfy al1 the known physical and thermodynamic relationships among these properties. The quantities $\Delta_{f} H^{\circ}, \Delta_{f} G^{\circ}$, and $S^{\circ}$ at 298.15 K satisfy the relation:

$$
\Delta_{f} G^{\circ}=\Delta_{f} H^{\circ}-T \Delta_{f} S^{\circ} .
$$

to the precision given. The special case of solutions is discussed in section 2.4. Furthermore the calculated value of aniy thermodynamic quantity for a reaction is independent of the path chosen for the evaluation. 
In some cases newer data may have become available on certain substances after the values were selected for these tables. Because of the need to maintain the internal consistency of the tables, it is not always possible to incorporate these newer data into the tables without a detailed analysis of the effect of such a change. Unless great care is used, relatively significant errors in calculated values of $\Delta H^{\circ}$ or $\Delta G^{\circ}$ for specific reactions may result from the introduction of such data. See section I-4.2.

There are, however, some substances [limited to the sodium and potassium salts of $\mathrm{SO}_{4}^{-2}(\mathrm{a}), \mathrm{HCO}_{3}^{-}(\mathrm{a})$ and $\left.\mathrm{CO}_{3}^{-2}(\mathrm{a})\right]$ for which the G-H-S relationship has been relaxed, so that $\Delta_{f} G^{\circ}(c)$ does not equal $\Delta_{f} H^{\circ}(c)-T \Delta_{f} S^{\circ}(c)$ within the stated uncertainty. These are designated in the tables (section II) by the statement:

G-H-S constraint has been relaxed.

The rationale is as follows:

Newer data on the entropies of solution and Third Law entropies of a number of salts have led to significantly different values of the entropies of these ions from those reported in TN 270-3 [81NBS] and used throughout the series. In order to retain the basic principle of those tables insofar as possible, namely that the values in the tables should yield "best" values for thermodynamic processes, values for $\Delta_{f} H^{\circ}$, and $\Delta_{f} G^{\circ}$ for the alkali metal salts have been selected such that the "best" values of $\Delta H^{\circ}$ and $\Delta G^{\circ}$ for the ideal solution process are maintained. The values of $S^{0}$ for the salts are selected from measurements independent of the solution process. In these cases the following thermodynamic relation:

$$
\Delta S^{\circ}(\text { solution })=\left[\Delta H^{\circ}(\text { solution })-\Delta G^{\circ}(\text { solution })\right] / T
$$

will hold for the ideal solution process but the following relation may not be satisfied within the expected precision of the individual values of $S^{\circ}$ :

$$
\Delta S^{\circ}(\text { solution }) \neq \sum_{\text {ions }} S^{\circ}(a)-S^{\circ}(c)
$$


Those processes affected in section II are designated.

See TN 270-8 [81NBS] for further details.

\subsection{Uncertainties}

The uncertainty in any value in the tables in section II depends on the uncertainties of all the determinations in the total chain of reactions used to establish the value.

In general, when uncertainties are not stated explicitly certain rules have been followed with respect to the number of significant figures recorded. Values are tabulated such that the overall uncertainty lies between 2 and 20 units of the last figure. On the other hand, values are given so that the experimental data from which they are derived may be recovered with an accuracy equal to that of the original quantities. Thus the number of significant figures for any one value in the tables need not represent the absolute accuracy of that value. For solutions of varying composition, values are frequently tabulated to more figures to make possible the recovery of enthalpies of solution and dilution.

Each value, then, calculated for a process from the property values is given to one more decimal place than the property value with the least significant value.

\subsection{Relationship to 0ther Tables of Thermodynamic Properties and Processes}

The chemical thermodynamic properties and processes in the present tables may be combined with those published in TN 270 [81NBS] in order to calculate the change in another process. However we recommend against these values being combined with those in any other tabulation or with a property reported in an original research paper.

There are several reasons for avoiding the combination of thermochemical data from more than one source table. The most important is that different 
large-scale tables use different thermochemical properties of formation for substances that are ubiquitous in thermochemical measurements. Outstanding examples are the common inorgaric acids and their ions. Another reason is that the groups preparing different tables may have relied on different measurements as the basis for selecting property values.

It is difficult to predict a priori how a change in one selected formation property or in a process would affect values assigned to other substances or processes because of the way these are linked by complex networks. In general, it may be expected that the advantage of internal consistency of a table will be lost if values from several sources are combined and that experimental measurements may be reproduced poorly.

No general, simple algorithm can be suggested for overcoming this problem. If it becomes necessary to extend a table of data to substances other than those tabulated, the user is advised to consult the group that prepared the table about the procedure that he plans to use. 


\section{Table I-1: Physical State Conventions}

The following conventions are used to designate the physical state of a substance. This information appears in a parenthetical expression appended to the molecular formula.

Basic Symbols

(g)

(gs)

(c)

(cs)

(am)

\section{Explanation}

Gaseous, e.g., $\operatorname{HCl}(g)$

Gaseous reference standard state for an

elenient, e.g., $\mathrm{O}_{2}$ (gs) for $\mathrm{O}_{2}(\mathrm{~g})$

Crystalline, e.g., $\mathrm{NH}_{4} \mathrm{Cl}(\mathrm{c})$

Crystalline reference standard state for an

element, e.g., $\mathrm{Rb}(\mathrm{cs})$ for $\mathrm{Rb}(\mathrm{c})$

Liquid, e.g., $\mathrm{H}_{2} \mathrm{O}(\mathrm{T})$

Liquid reference standard state for an

element, e.g., $\mathrm{Br}_{2}(1 \mathrm{~s})$ for $\mathrm{Br}_{2}(1)$

Amorphous

GTassy

Hypothetical standard state of the ideal

aqueous solution at unit activity. For a

neutral electrolyte the value of a property

is equal to the algebraic sum of the values

for the ions assumed to constitute the

molecule of the electrolyte, e.g.,

$\mathrm{HCl}(\mathrm{a})=\mathrm{H}^{+}(\mathrm{a})+\mathrm{Cl}^{-}(\mathrm{a})$. For an ionic species

this notation is commonly used to refer to the

undissociated ion as written. e.g., $\mathrm{HSO}_{4}{ }^{-}(\mathrm{a})$. 

aqueous solution at unit activity of the undissociated (non-ionized) species, e.g., $\mathrm{HF}(\mathrm{ao}), \mathrm{HF}_{2}^{-}(\mathrm{ao})$. May also be used whenever the designation (a) could be ambiguous. Note that the descriptions $\mathrm{HSO}_{4}^{-}(\mathrm{a})$ and $\mathrm{HSO}_{4}^{-}(\mathrm{aO})$ are equivalent, but that $\operatorname{HF}(a)$ and $\operatorname{HF}\left(a_{0}\right)$ are not. 


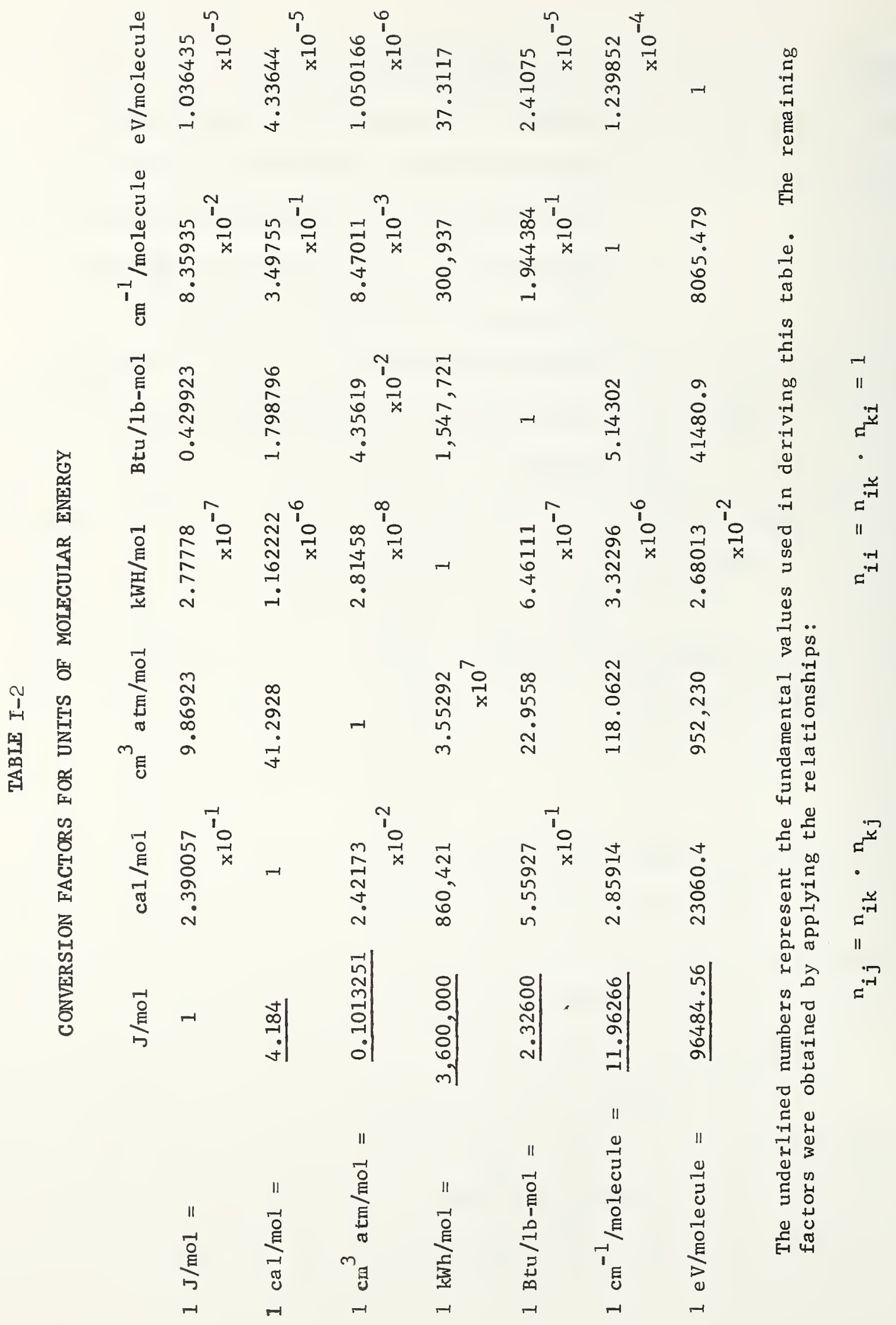


II Tables for the Thermochemical Property Values of Substances

The values tabulated are $\Delta_{f} H^{\circ}, \Delta_{f} G^{\circ}$ and $H^{\circ}-H_{0}^{\circ}$ in $\mathrm{kcal} / \mathrm{mol}, S^{\circ}$ and $\mathrm{C}_{\mathrm{p}}^{\circ}$ in $\mathrm{cal} /(\mathrm{mol} \cdot \mathrm{K})$, all at $298.15 \mathrm{~K}$.

The tables are arranged in the following order:

Table II-1 The $0, \mathrm{H}, \mathrm{S}$, or $\mathrm{C}$ containing substances

II-2 The $\mathrm{Fe}-\mathrm{O}, \mathrm{H}, \mathrm{S}$, or $\mathrm{C}$ containing substances

II-3 The Mn - $0, H, S$, or $C$ containing substances

II-4 The Mg - O, H, S, or C containing substances

II-5 The $\mathrm{Ca}-\mathrm{O}, \mathrm{H}, \mathrm{S}$, or $\mathrm{C}$ containing substances

II-6 The $\mathrm{Na}-0, \mathrm{H}, \mathrm{S}$, or $\mathrm{C}$ containing substances

II-7 The $\mathrm{K}-0, \mathrm{H}, \mathrm{S}$, or $\mathrm{C}$ containing substances 


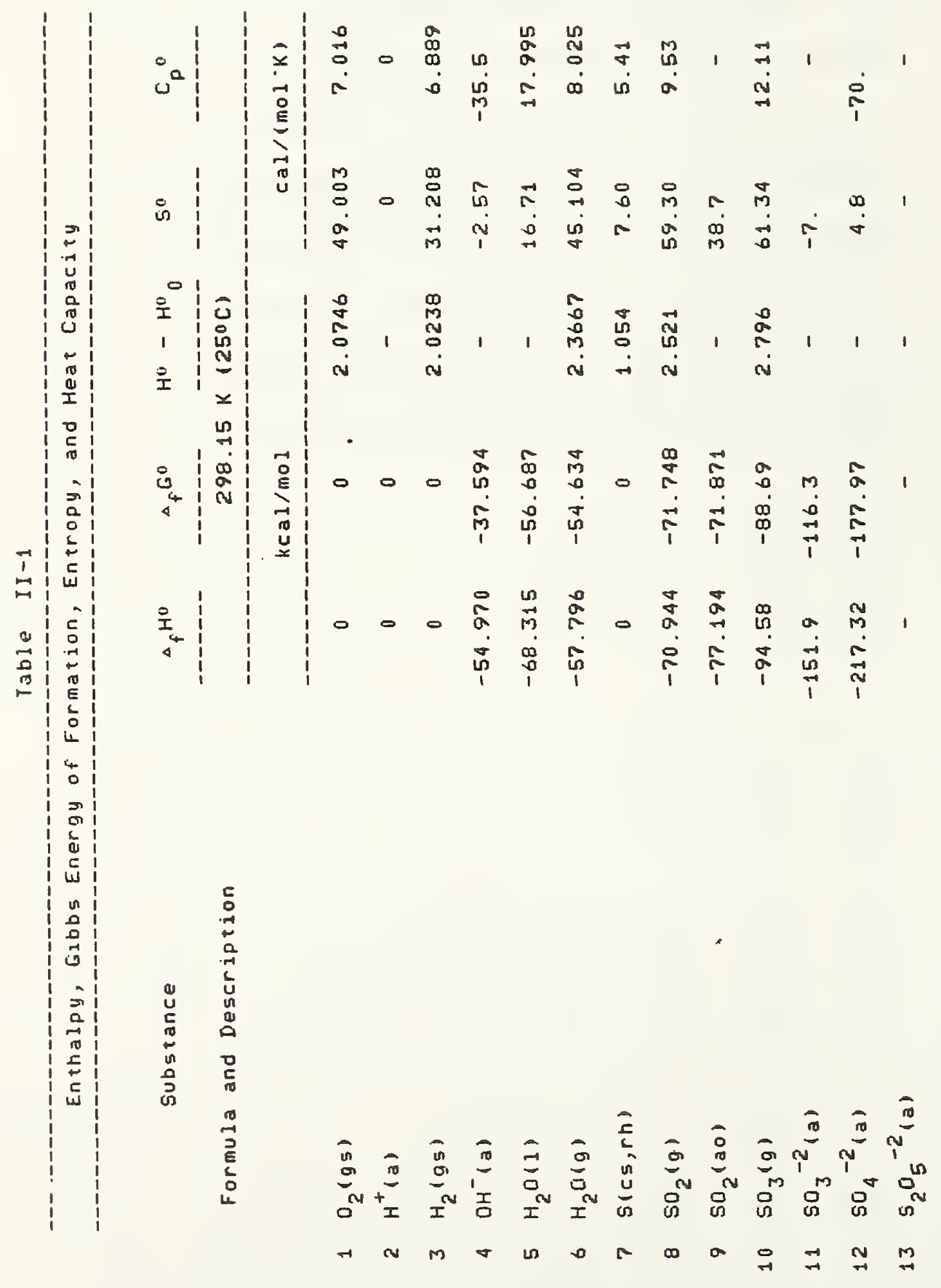




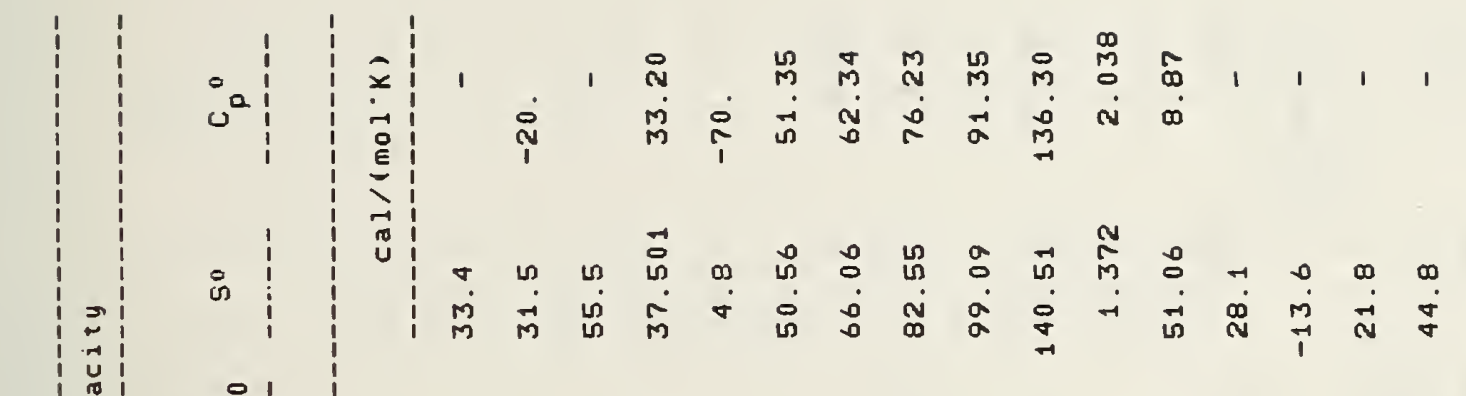

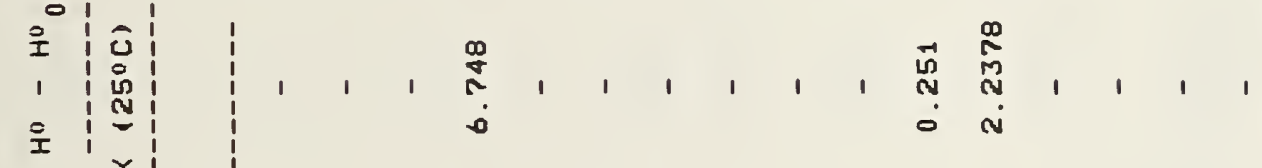

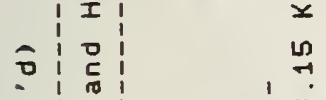

$$
\begin{aligned}
& \div 10 \\
& \text { c: } \\
& \begin{array}{rlll}
0 & 0 & 0 & 1 \\
1 & 2 & 1 \\
-1 & 0 & 1
\end{array}
\end{aligned}
$$

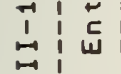

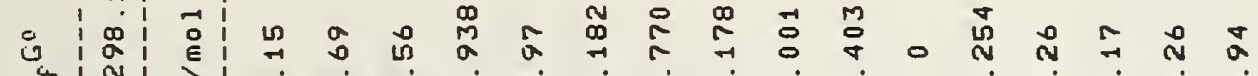

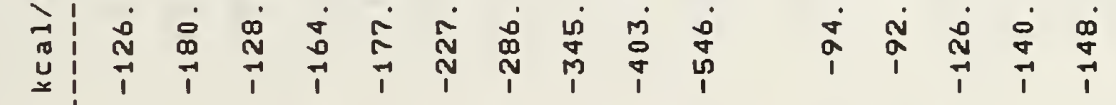

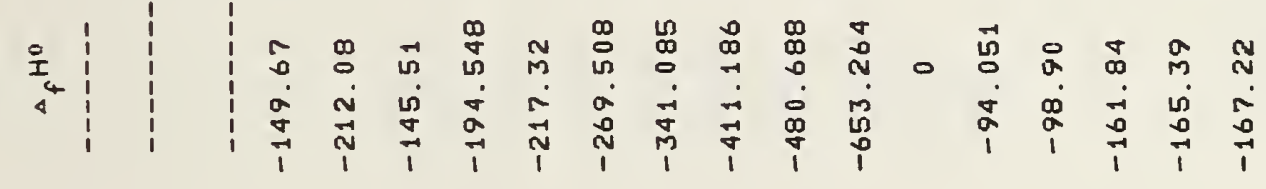

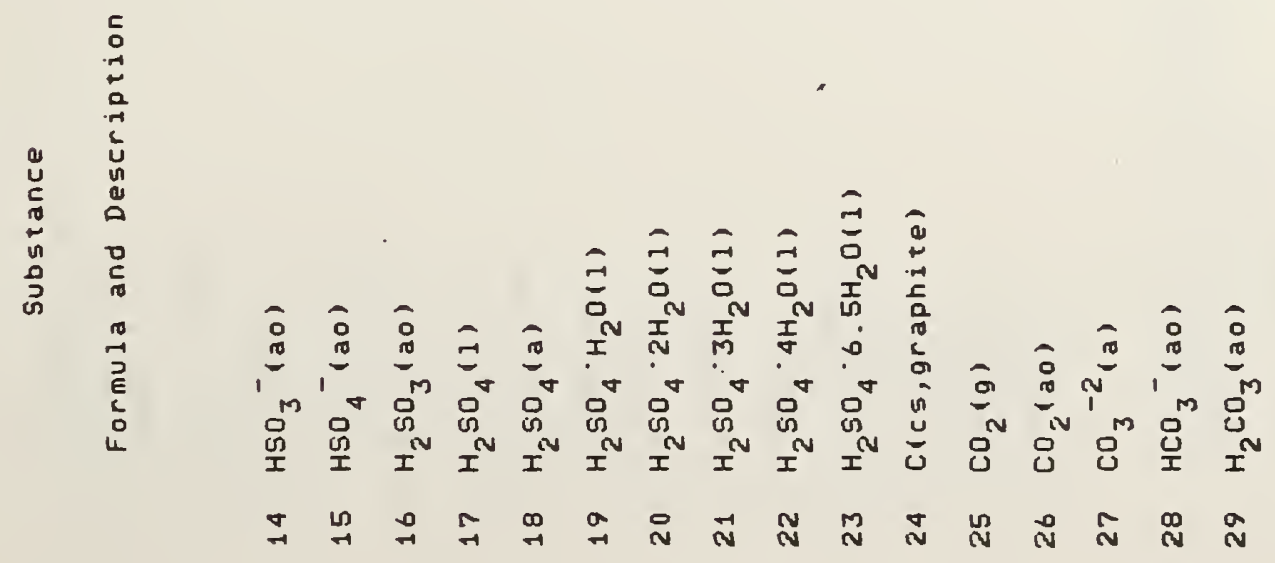




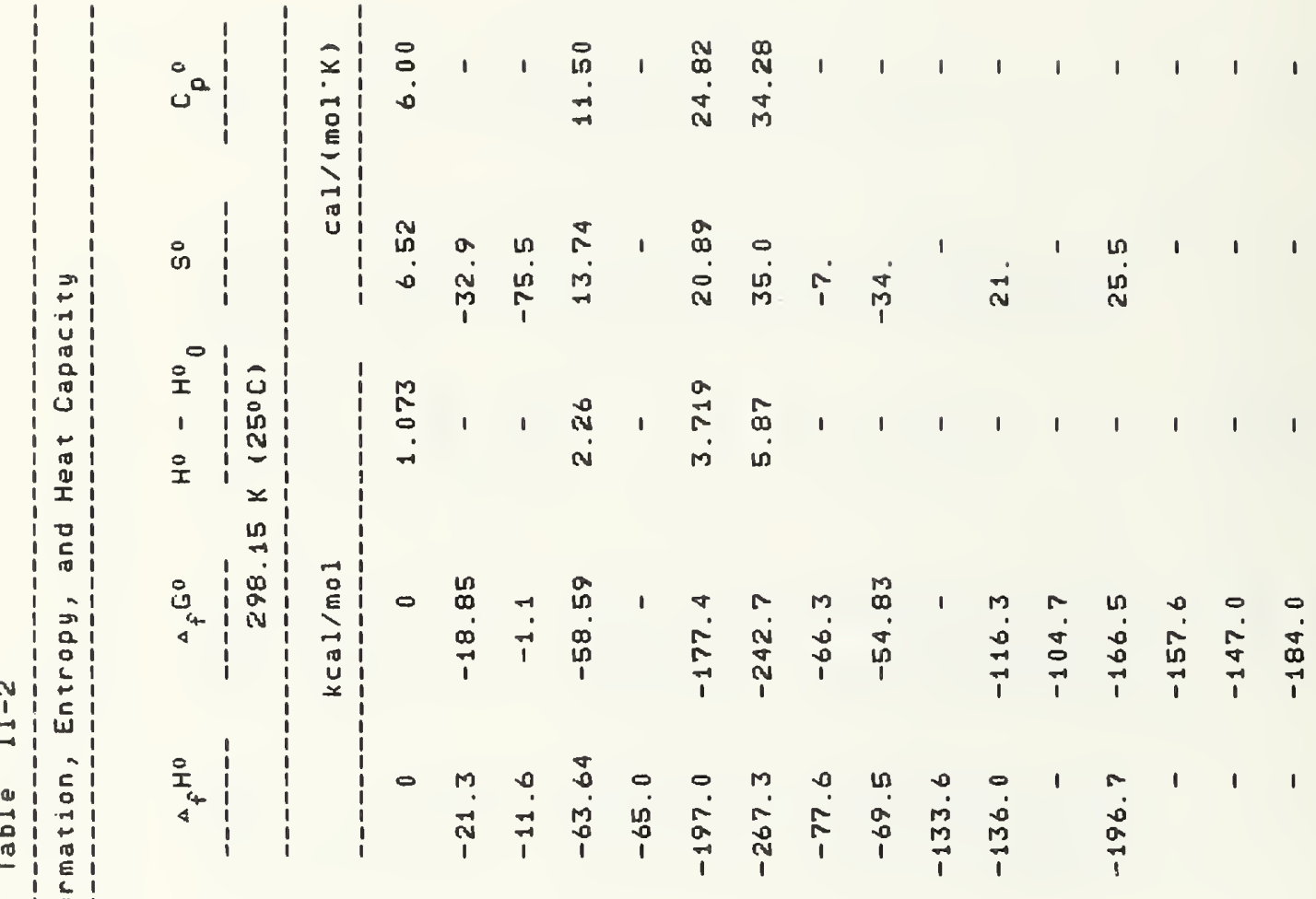

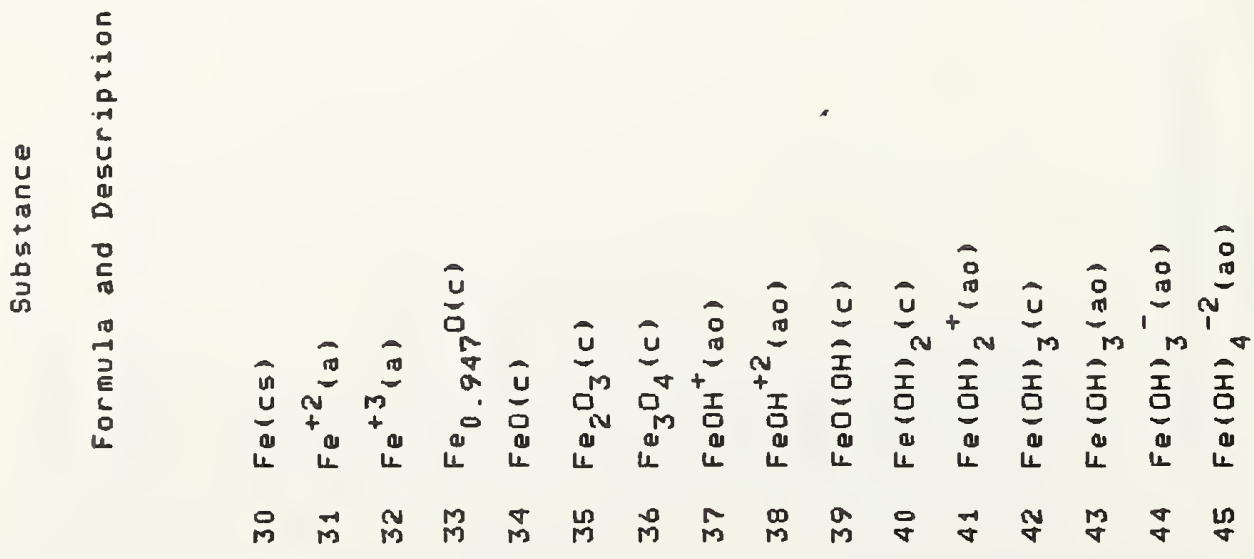




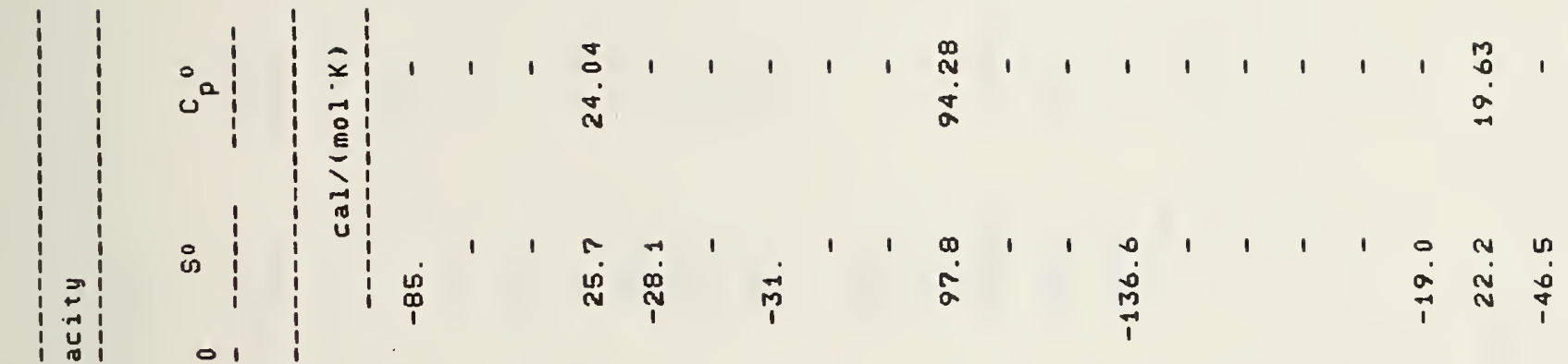

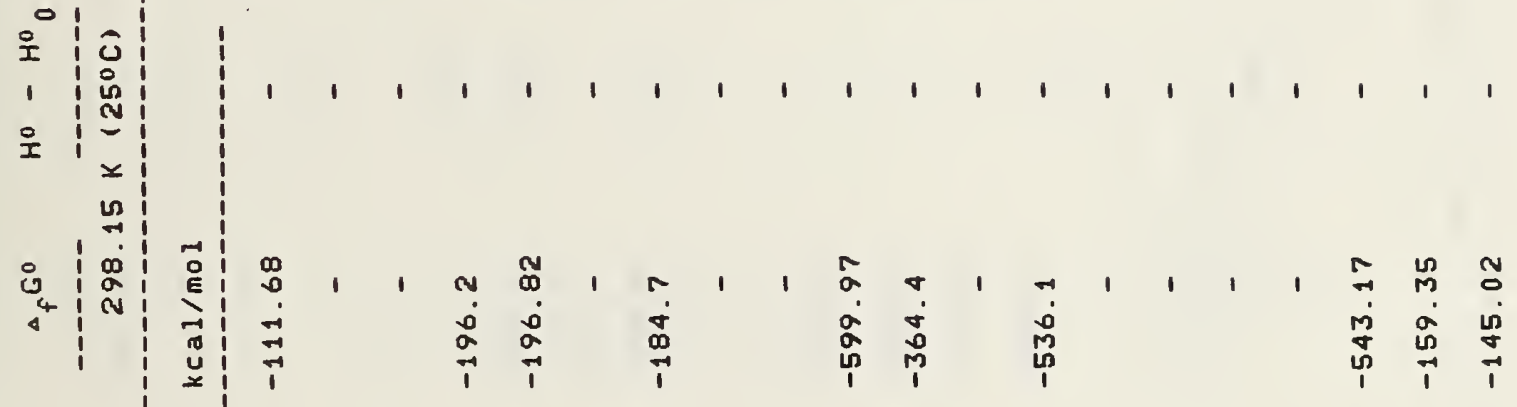

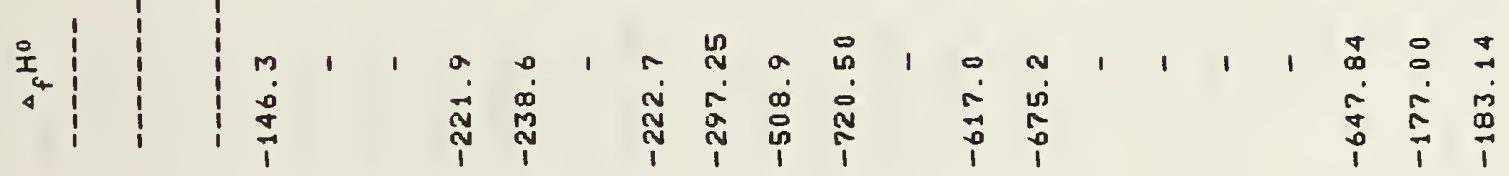

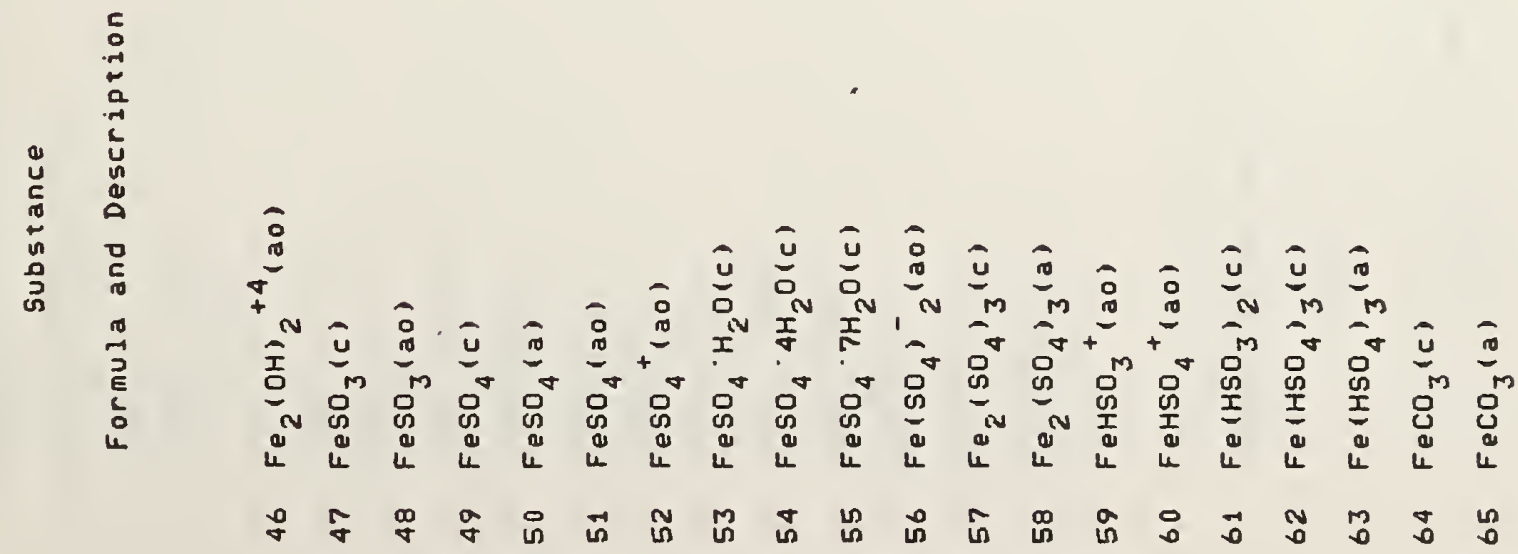




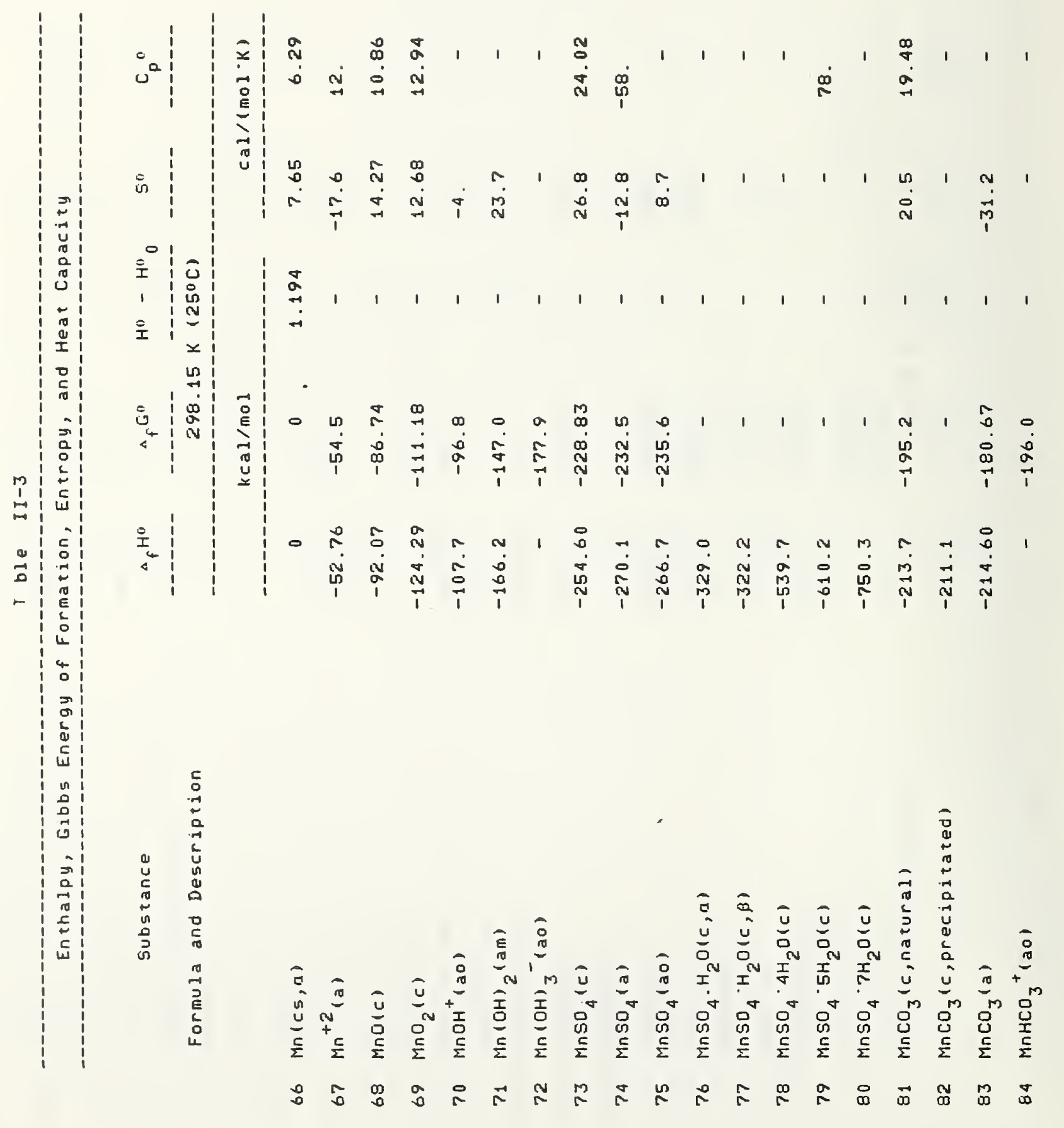




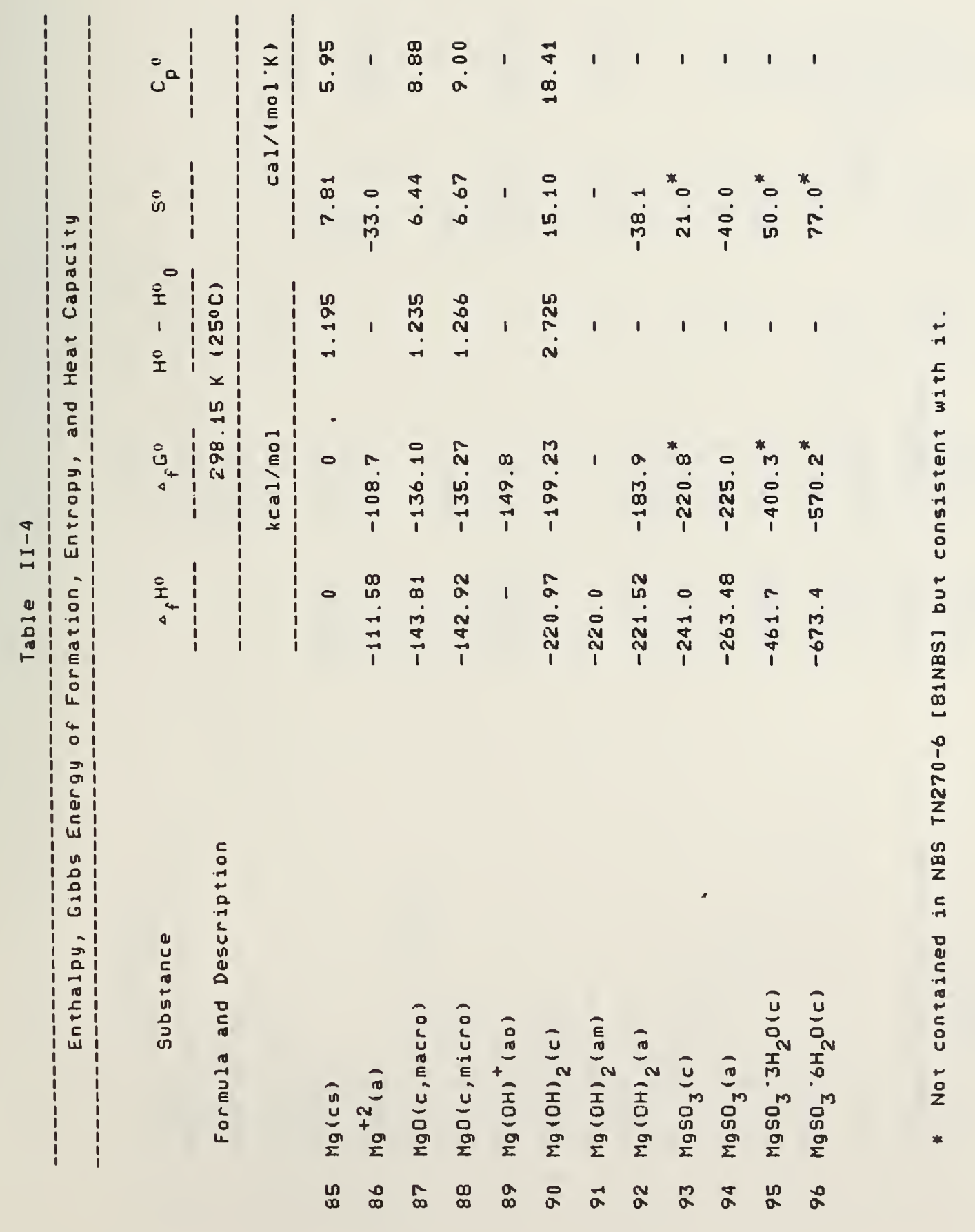




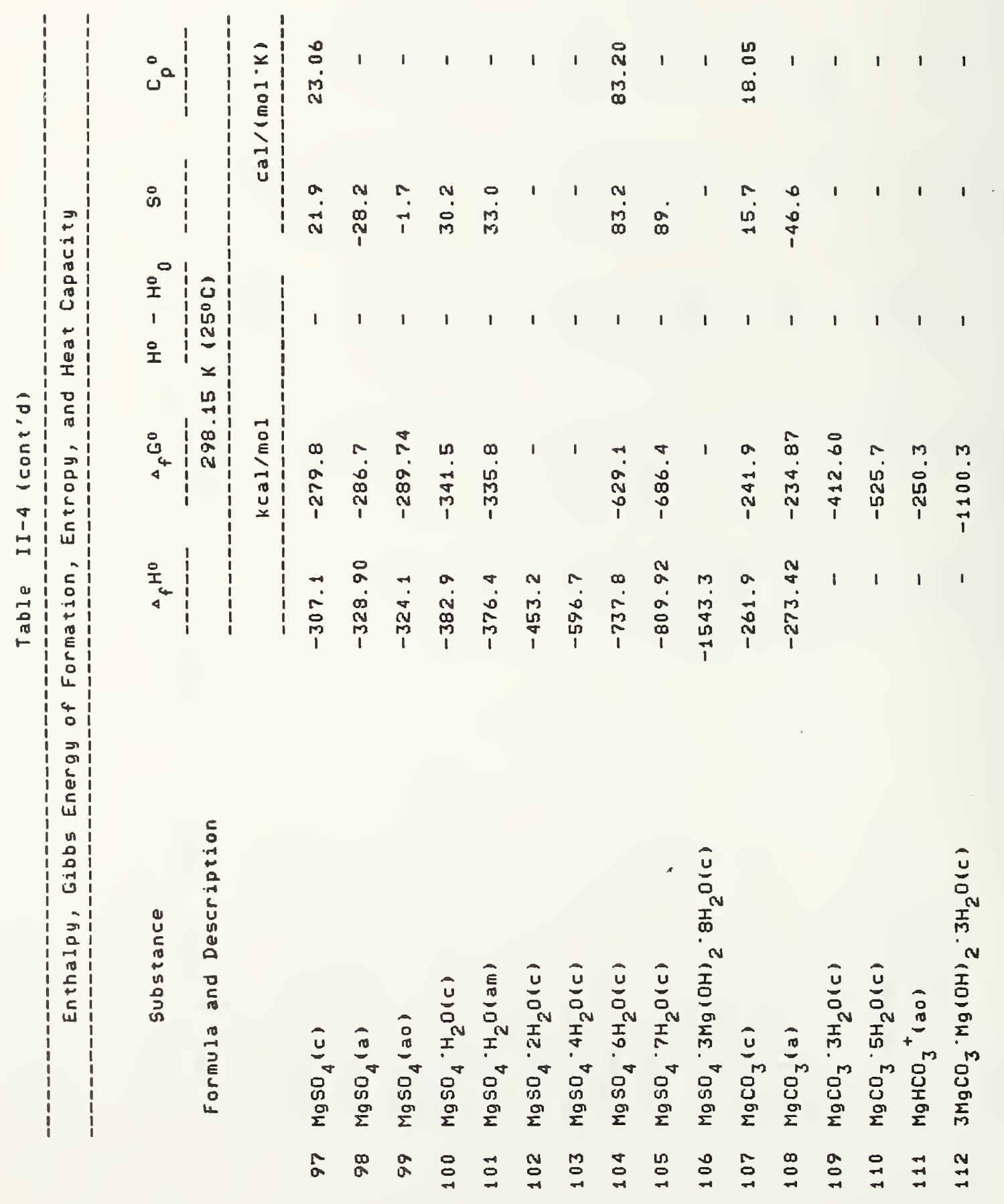




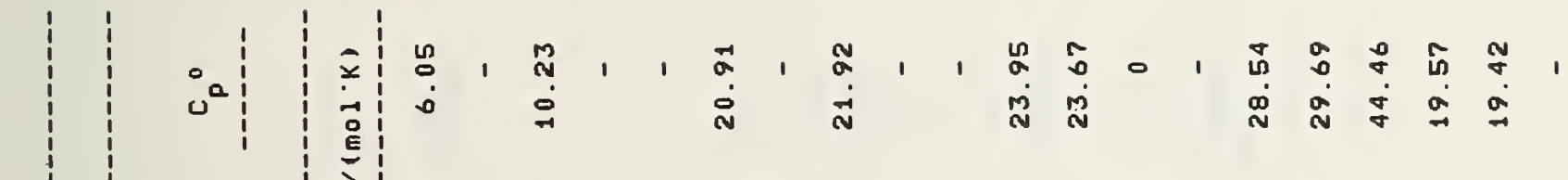

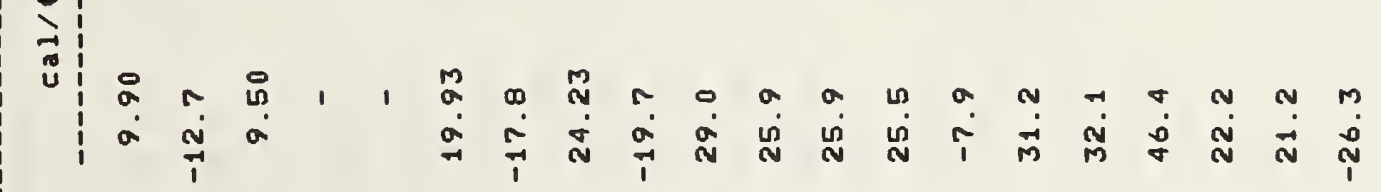

$$
\begin{aligned}
& \begin{array}{l:ll}
0 & 1 \\
\hline & 0 \\
1 & 1 & 0 \\
0 & 0
\end{array} \\
& \text { 일 }
\end{aligned}
$$

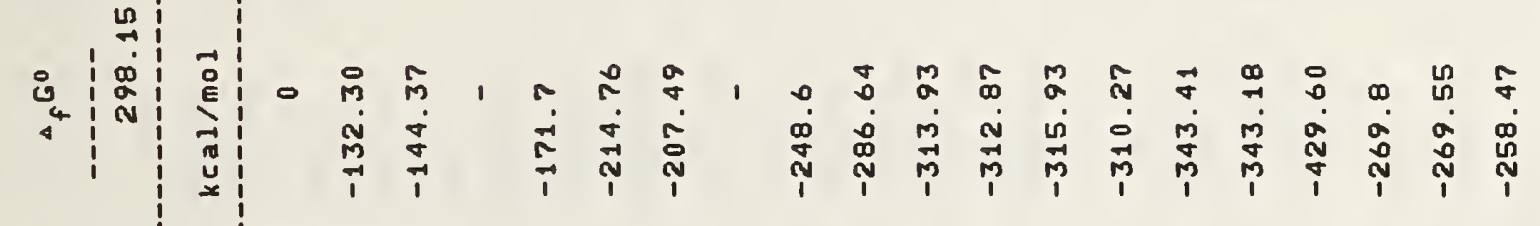

$$
\begin{aligned}
& \text { 4hinimimim }
\end{aligned}
$$

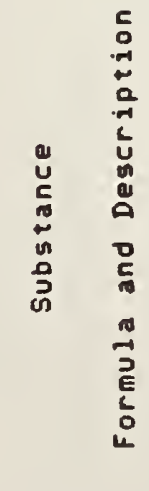

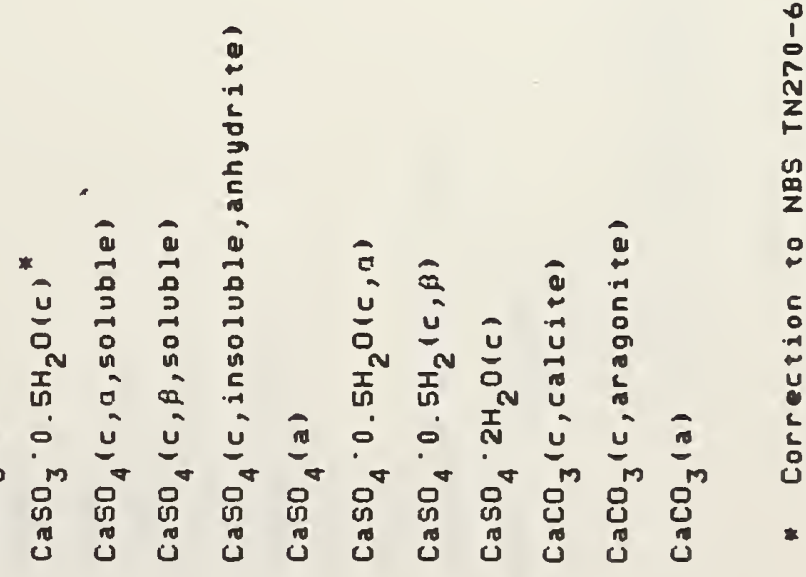

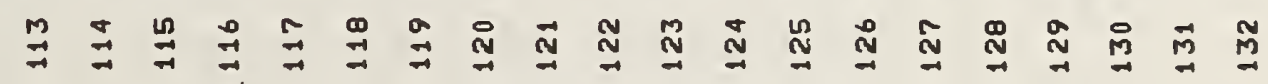




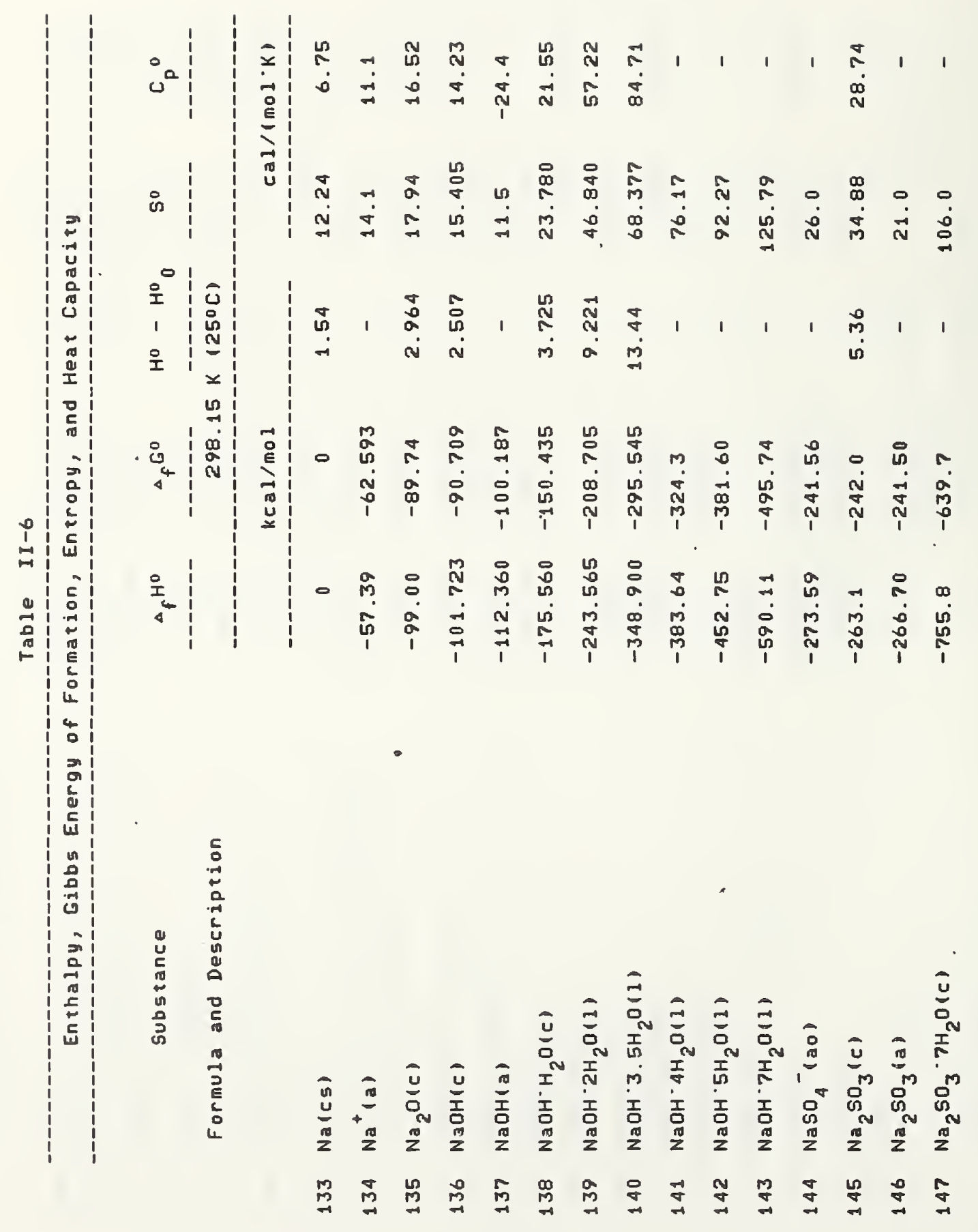




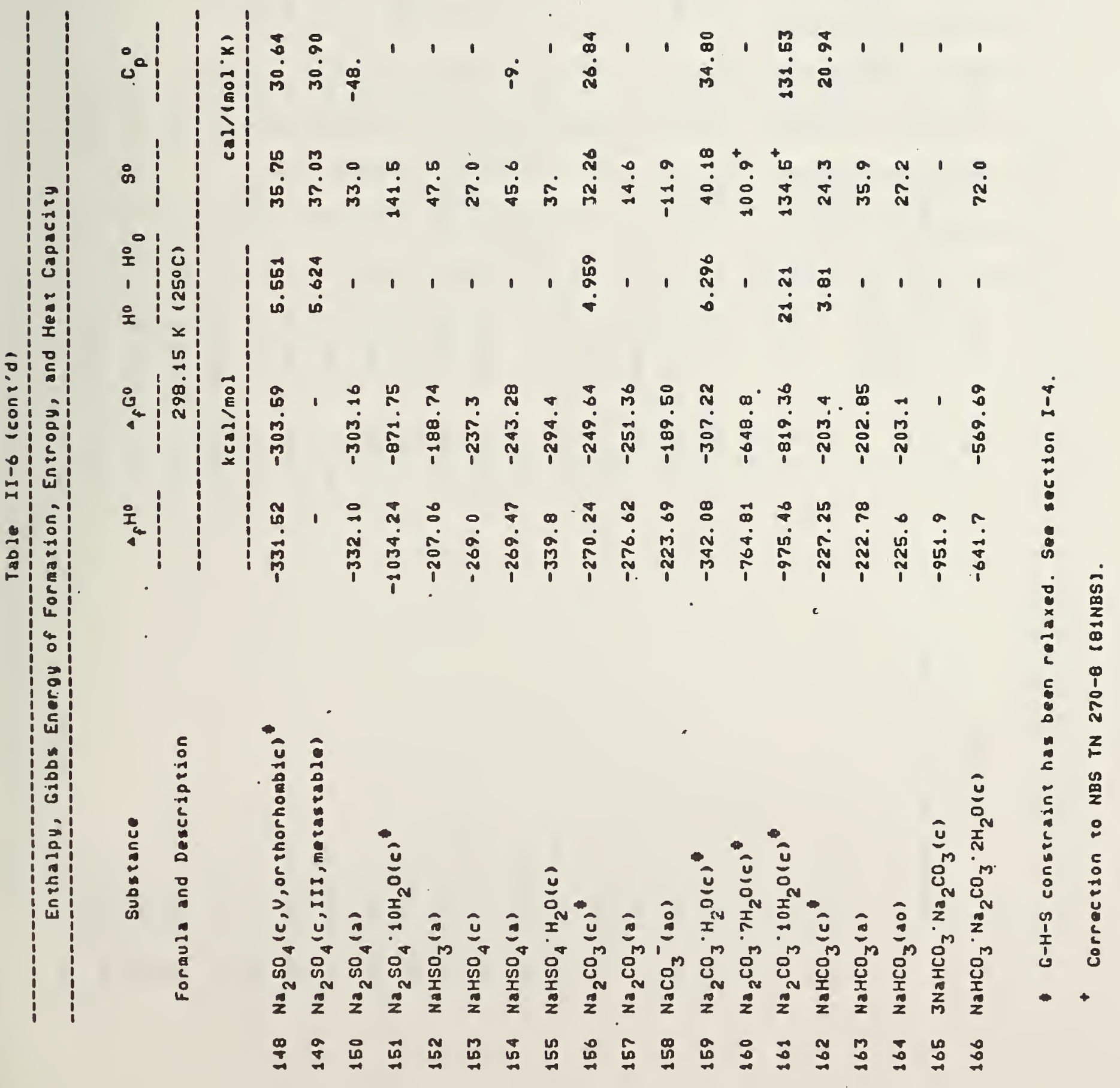




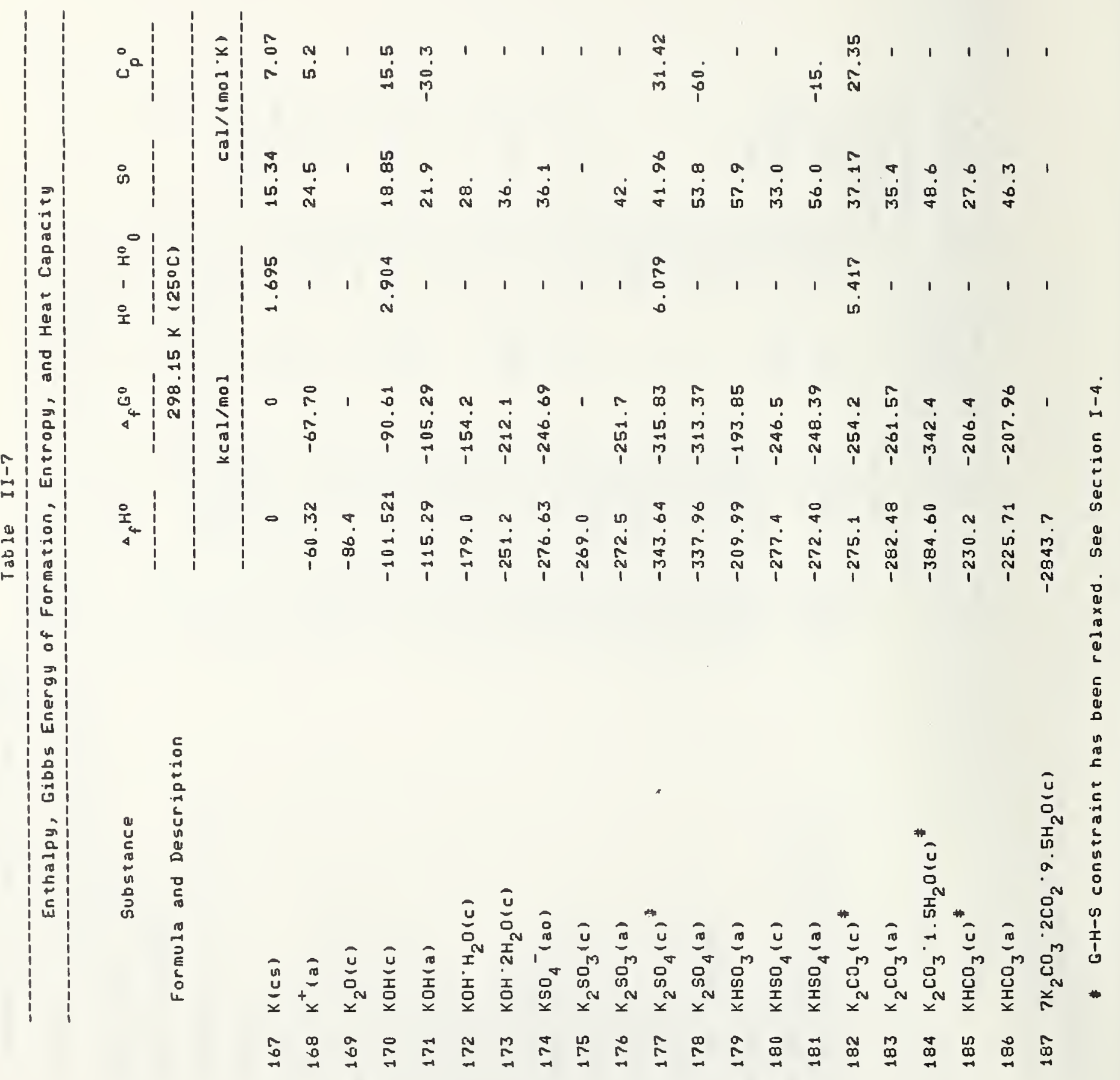


III Tables for the Thermochemical Values for Processes

The values tabulated are $\Delta H^{\circ}$ and $\Delta G^{\circ}$ in $\mathrm{kcal} / \mathrm{mol}, \Delta S^{\circ}$ and $\Delta C_{\mathrm{p}}^{\circ}$ in $\mathrm{cal} /(\mathrm{mol} \cdot \mathrm{K})$, and $\log K$ for the processes listed.

The tables are arranged in the following order:

Table III-l Processes involving $0, \mathrm{H}, \mathrm{S}$, or $\mathrm{C}$ containing substances

III-2 Processes involving $\mathrm{Fe}-\mathrm{O}, \mathrm{H}, \mathrm{S}$, or $\mathrm{C}$ containing substances

III-3 Processes involving $M n-0, H, S$, or $C$ containing substances

III-4 Processes involving $\mathrm{Mg}-\mathrm{O}, \mathrm{H}, \mathrm{S}$, or $\mathrm{C}$ containing substances

III-5 Processes involving $\mathrm{Ca}-\mathrm{O}, \mathrm{H}, \mathrm{S}$, or $\mathrm{C}$ containing substances

III-6 Processes involving $\mathrm{Na}-\mathrm{O}, \mathrm{H}, \mathrm{S}$, or $\mathrm{C}$ containing substances

III-7 Processes involving $\mathrm{K}-0, \mathrm{H}, \mathrm{S}$, or $\mathrm{C}$ containing substances 


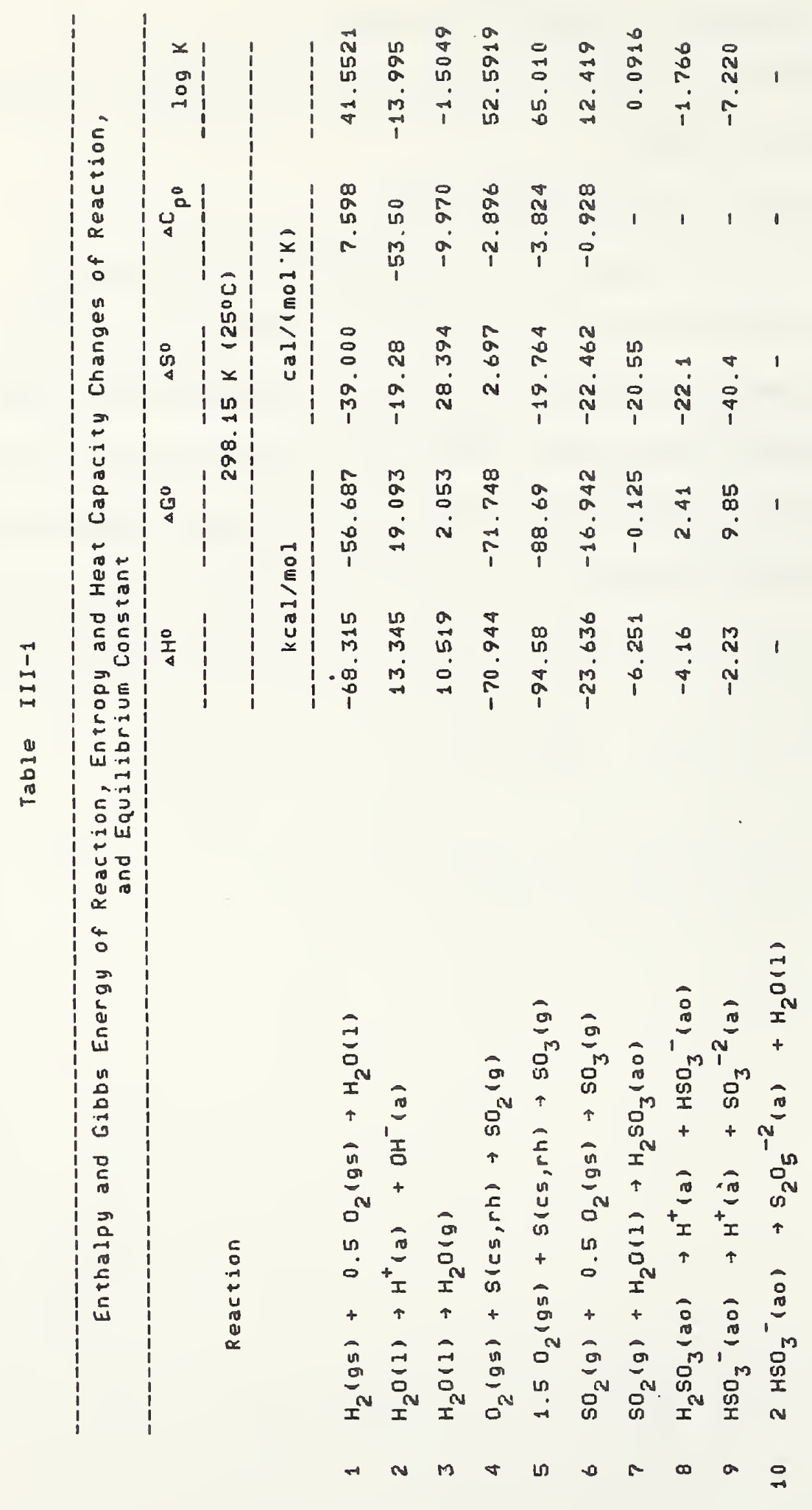




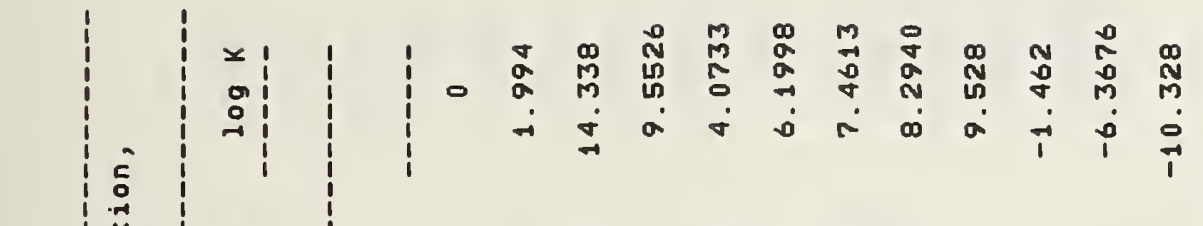

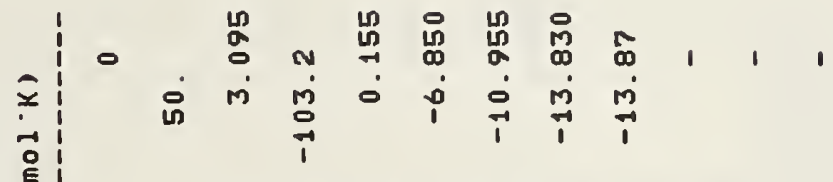

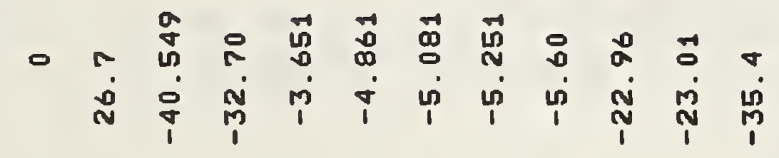

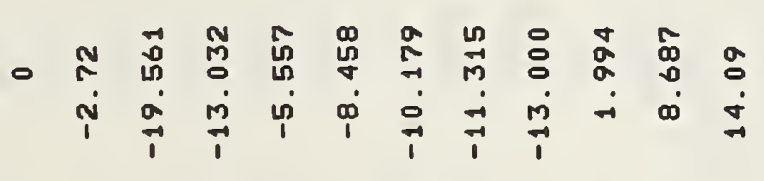

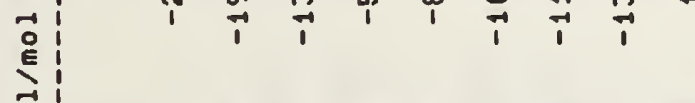

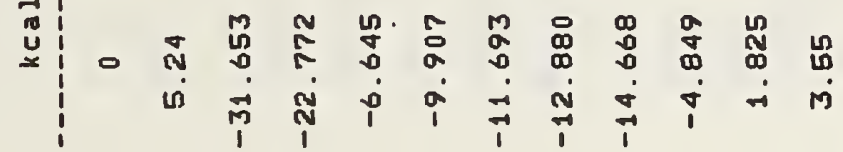

$$
\begin{aligned}
& \text { 它 }
\end{aligned}
$$

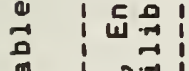

$$
\begin{aligned}
& \text { r }
\end{aligned}
$$

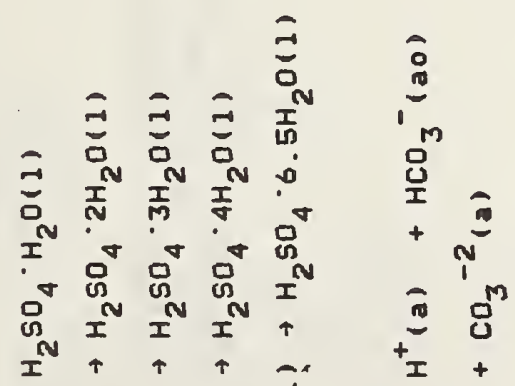

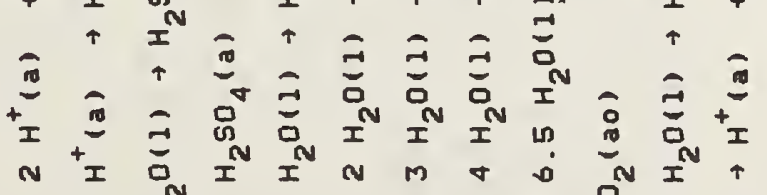

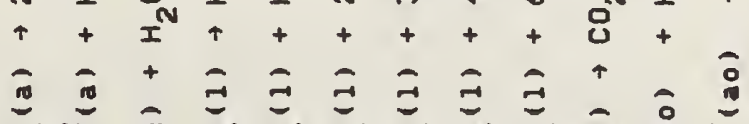

$$
\begin{aligned}
& \text { d }
\end{aligned}
$$

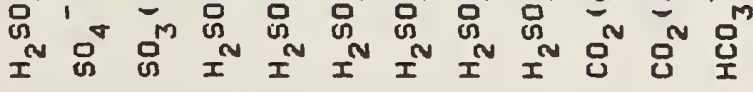

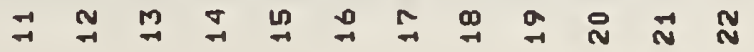




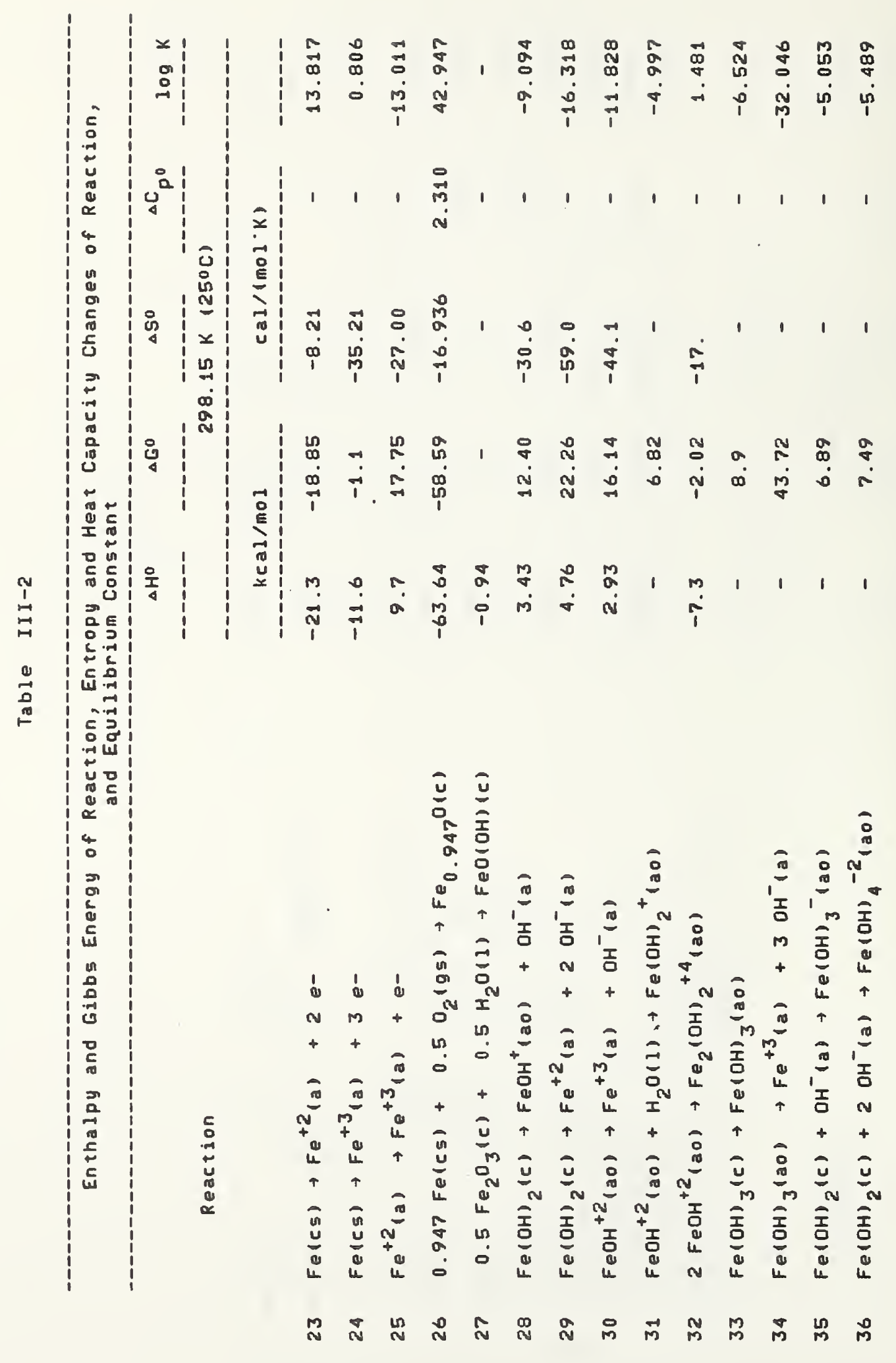




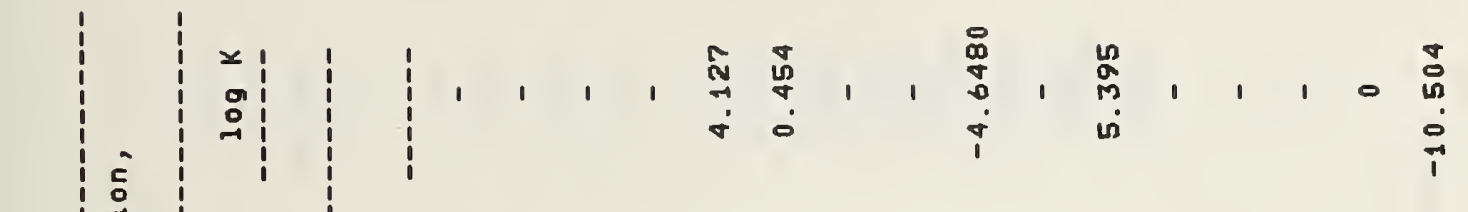

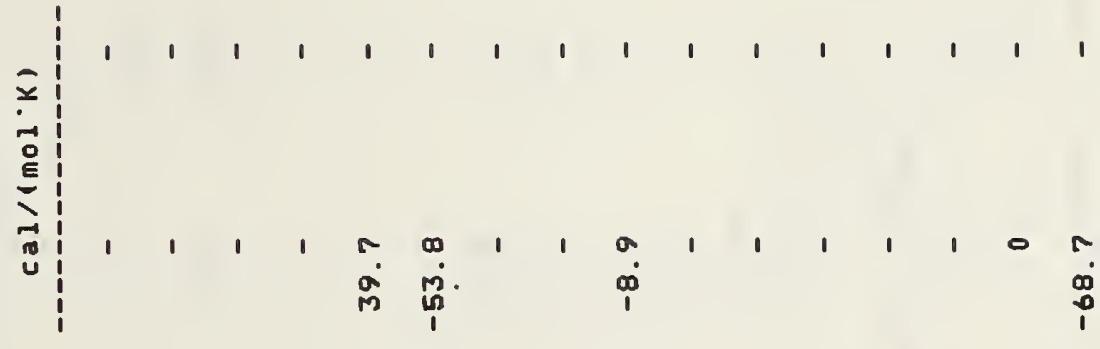

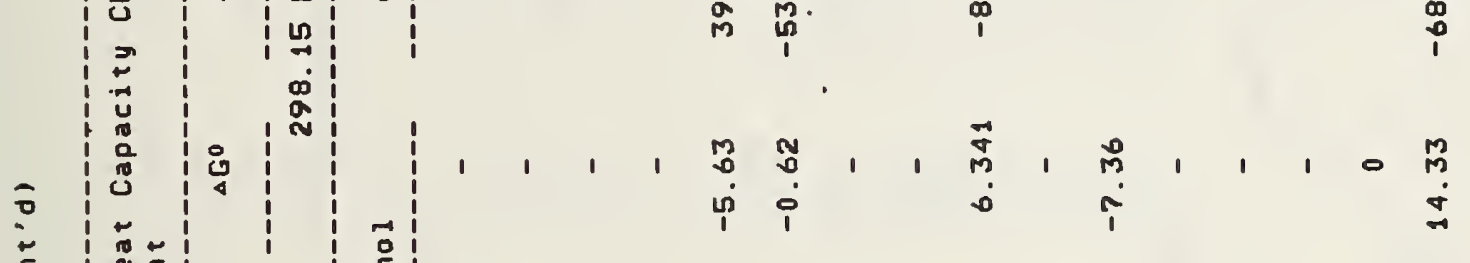

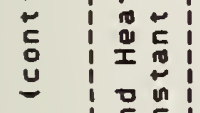

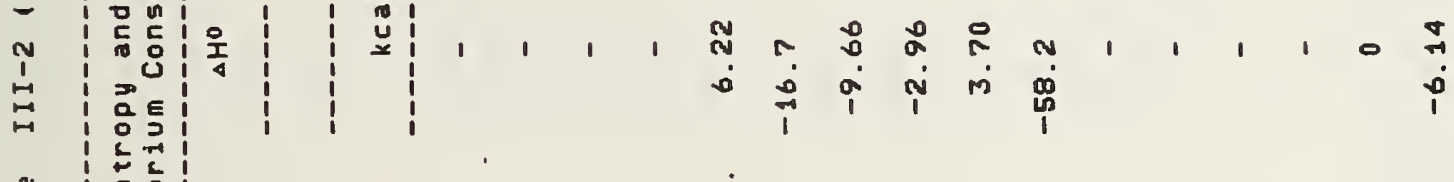

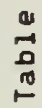

$$
\begin{aligned}
& \text { (1) } \\
& \text { 要要 } \\
& \text { प्र }
\end{aligned}
$$

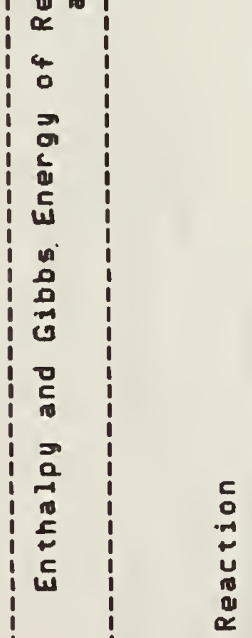

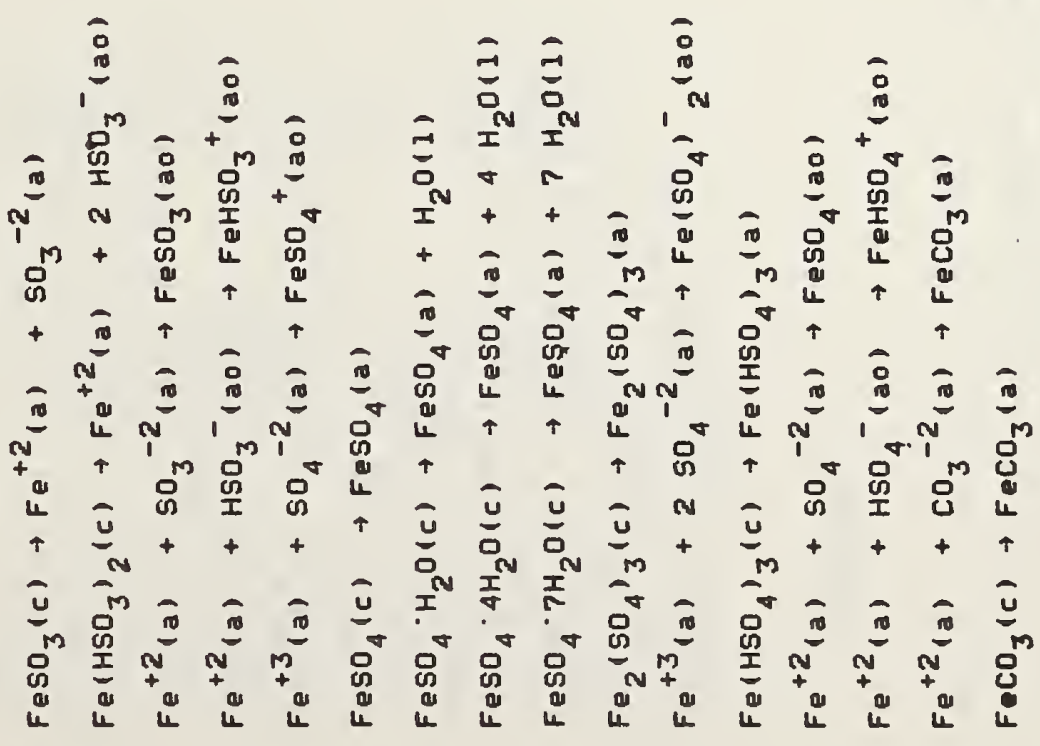

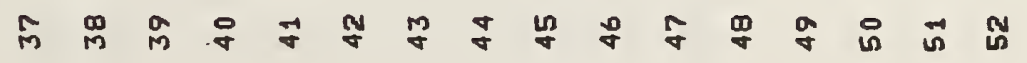




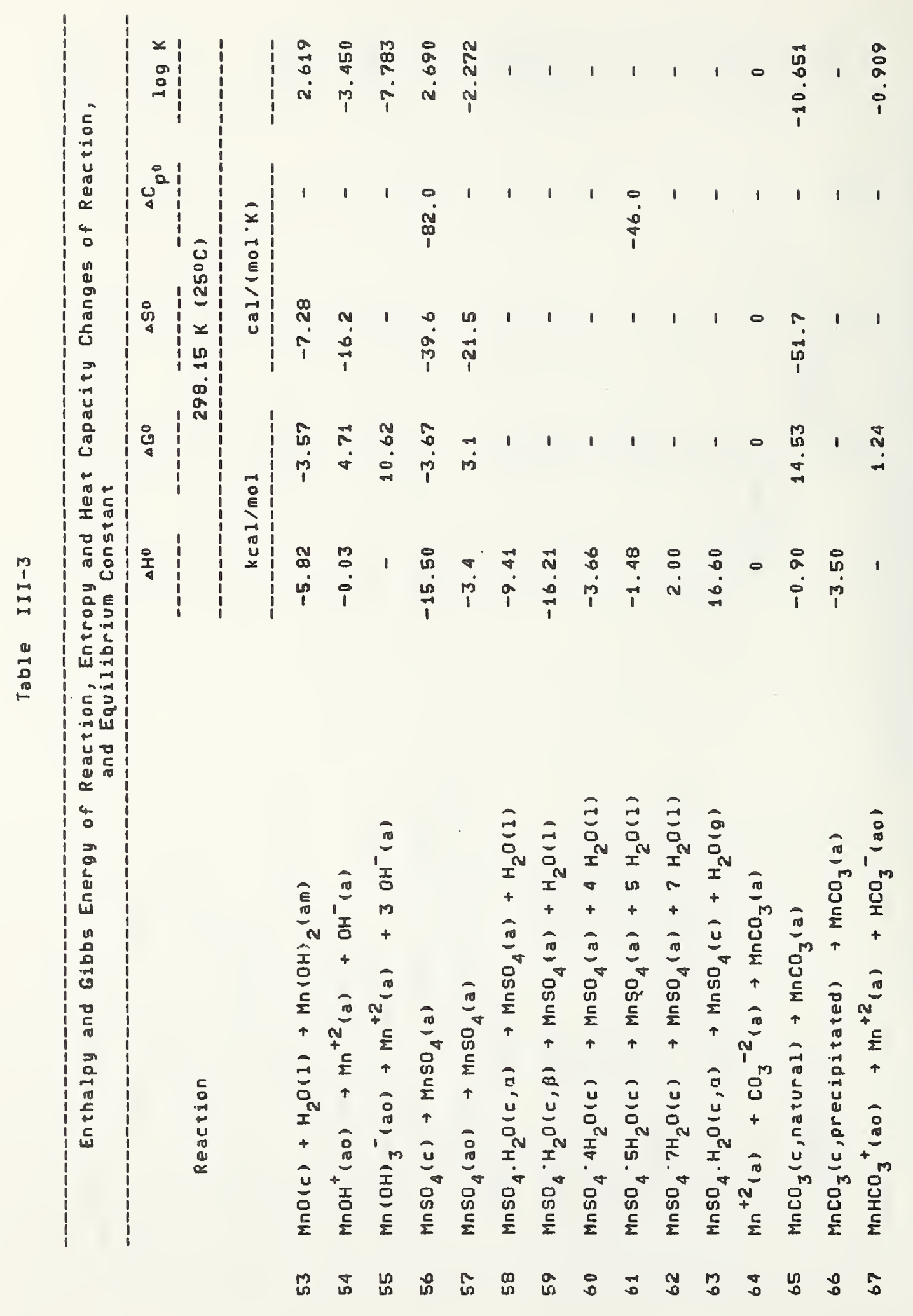




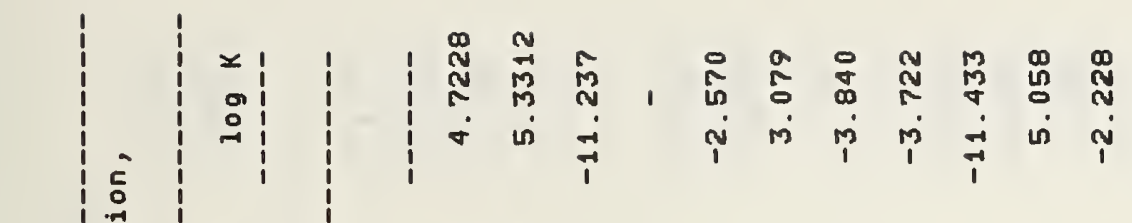

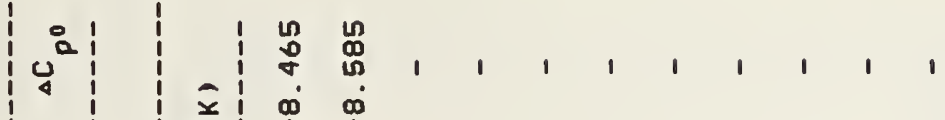

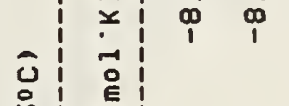

$$
\begin{aligned}
& \text { 는 }
\end{aligned}
$$

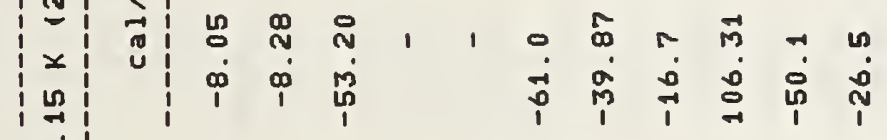

$$
\begin{aligned}
& \infty
\end{aligned}
$$

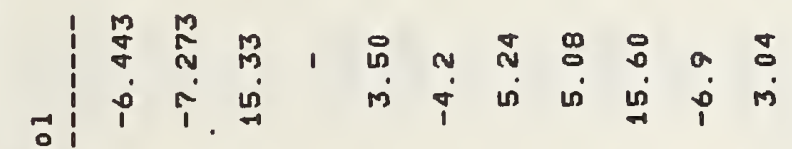

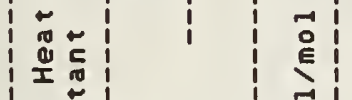

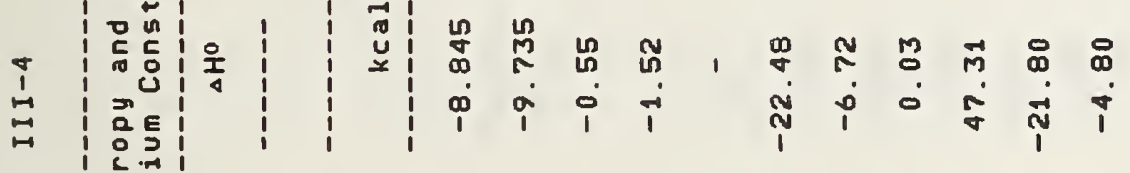

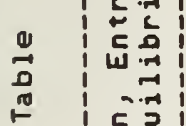

$$
\begin{aligned}
& \text { 究 } \\
& \text { 范 } \\
& \text { 足 } \\
& \text { 幽 } \\
& 4 \\
& 3 \\
& \text { a } \\
& \text { 它 } \\
& \text { in } \\
& \text { 告 } \\
& \text { 是 } \\
& \text { ? } \\
& \text { \% } \\
& \text { 品 } \\
& \text { 禹 } \\
& \text { 它 }
\end{aligned}
$$

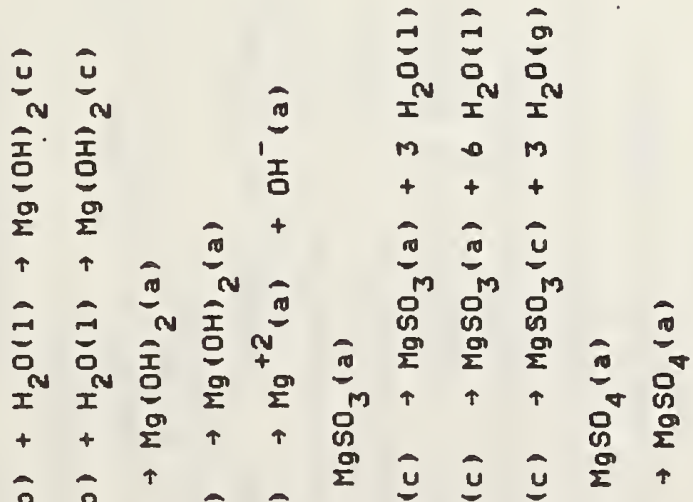

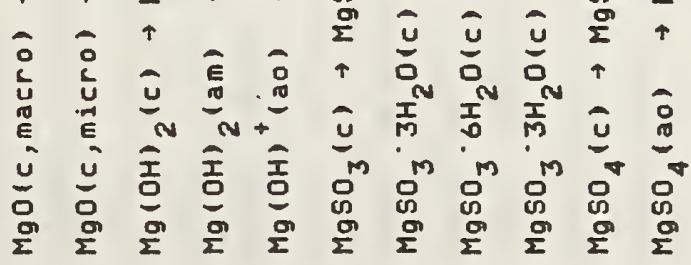

$$
\begin{aligned}
& \text { m d } 0 \text { 穴 }
\end{aligned}
$$




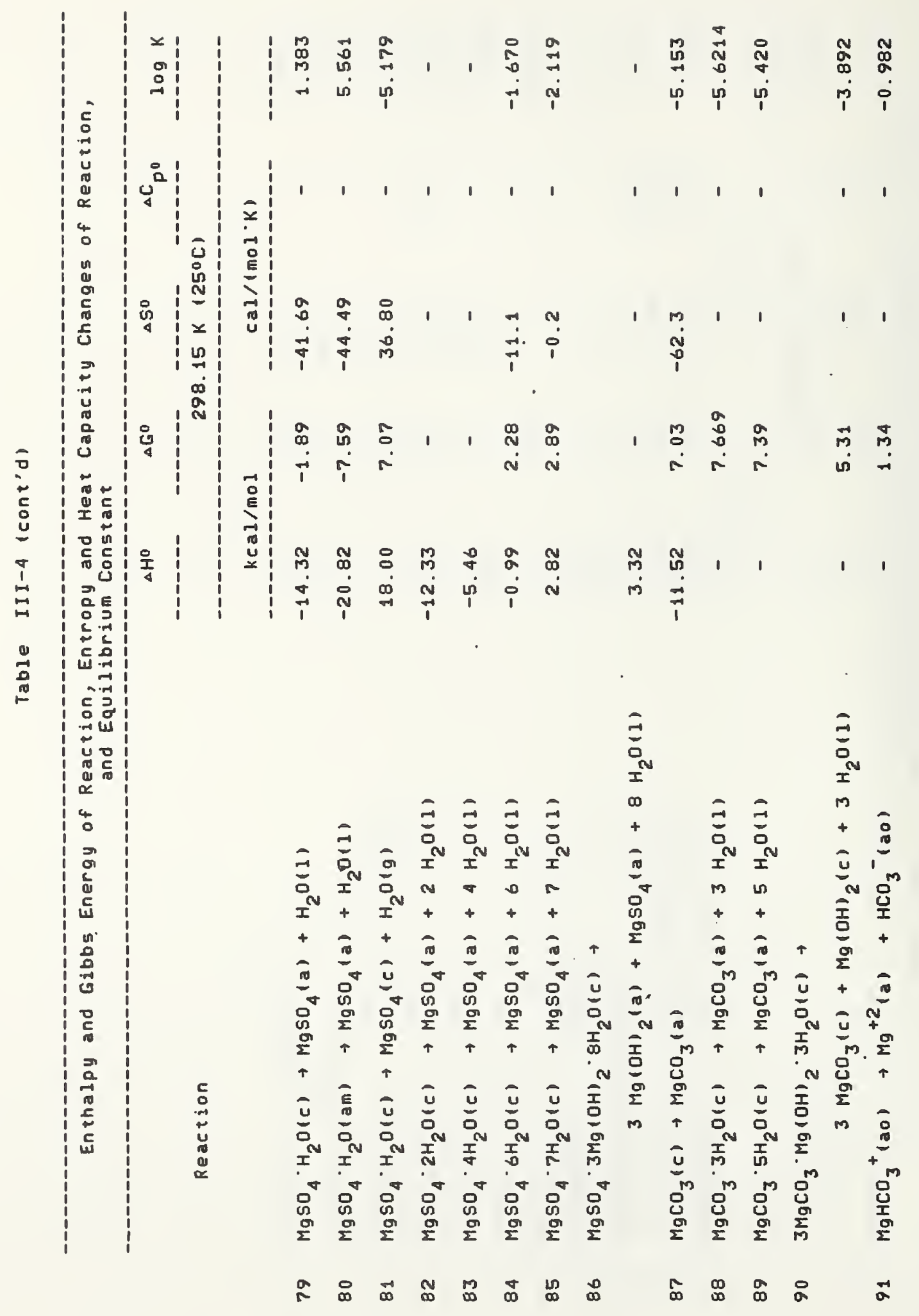




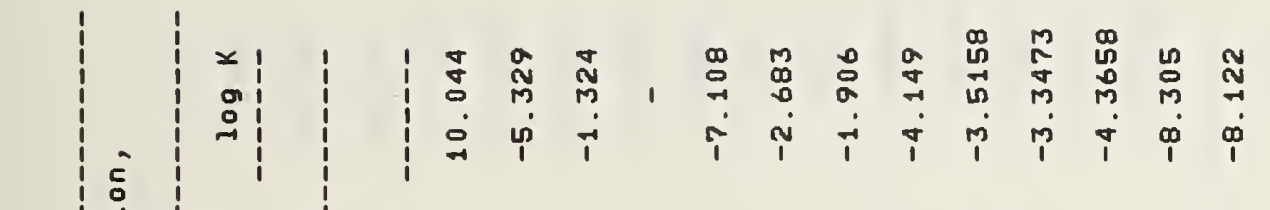

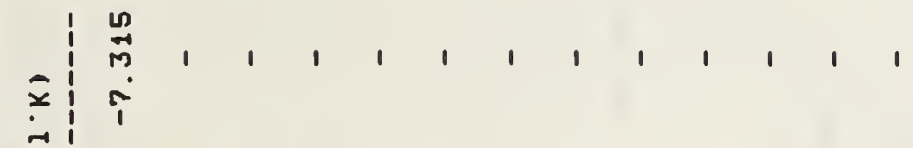

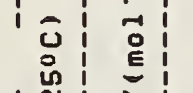

$$
\begin{aligned}
& \text { IN }
\end{aligned}
$$

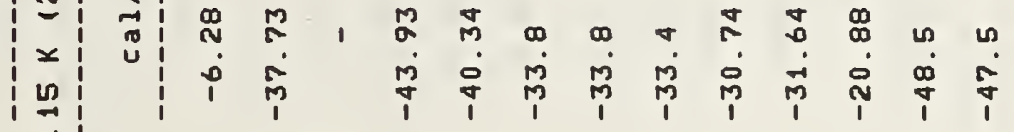

$$
\begin{aligned}
& \text { a }
\end{aligned}
$$

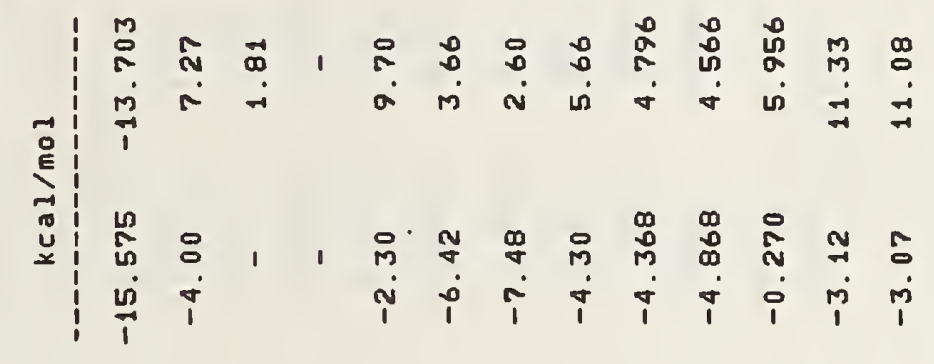

$$
\begin{aligned}
& \text { 足: }
\end{aligned}
$$

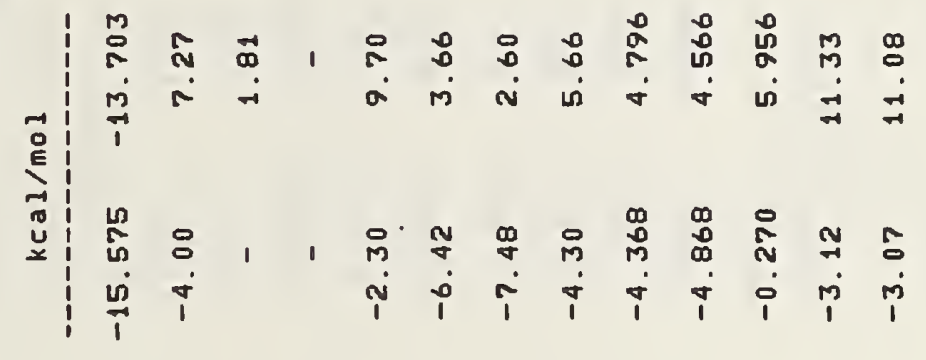

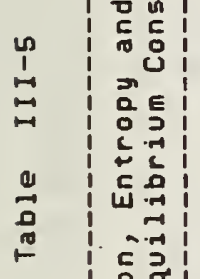

$$
\begin{aligned}
& \mid \begin{array}{cc}
5 & 2 \\
0 & 0 \\
0 & 0 \\
u & 0
\end{array} \\
& \text { 峁号 } \\
& 4 \\
& \text { a } \\
& \text { - }{ }^{2}
\end{aligned}
$$

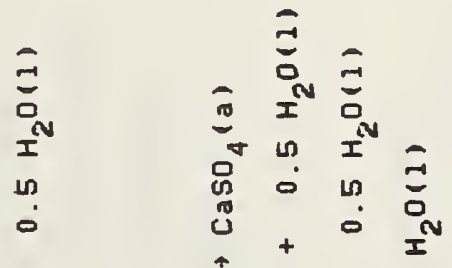

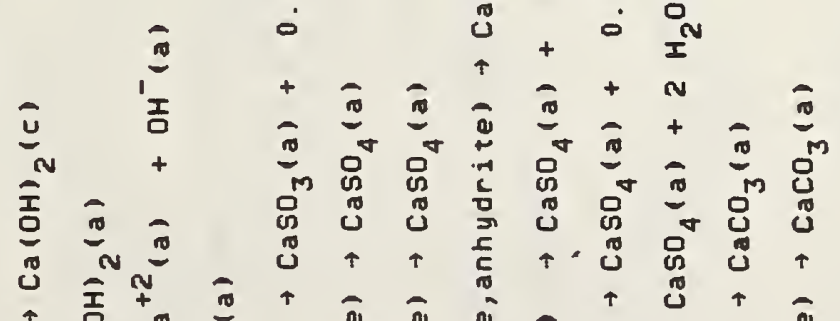

$$
\begin{aligned}
& \uparrow \text { 喜苾 }
\end{aligned}
$$

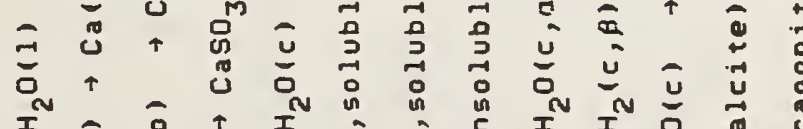

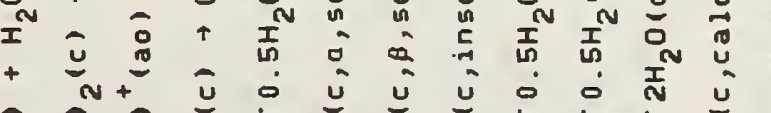

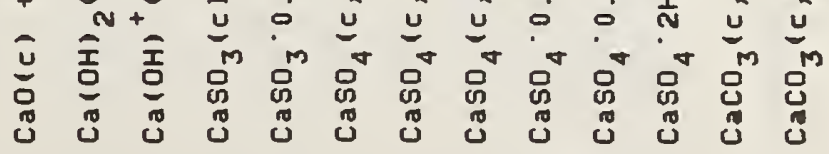

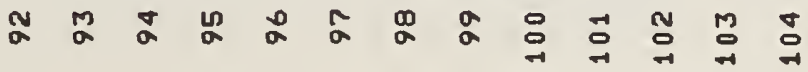




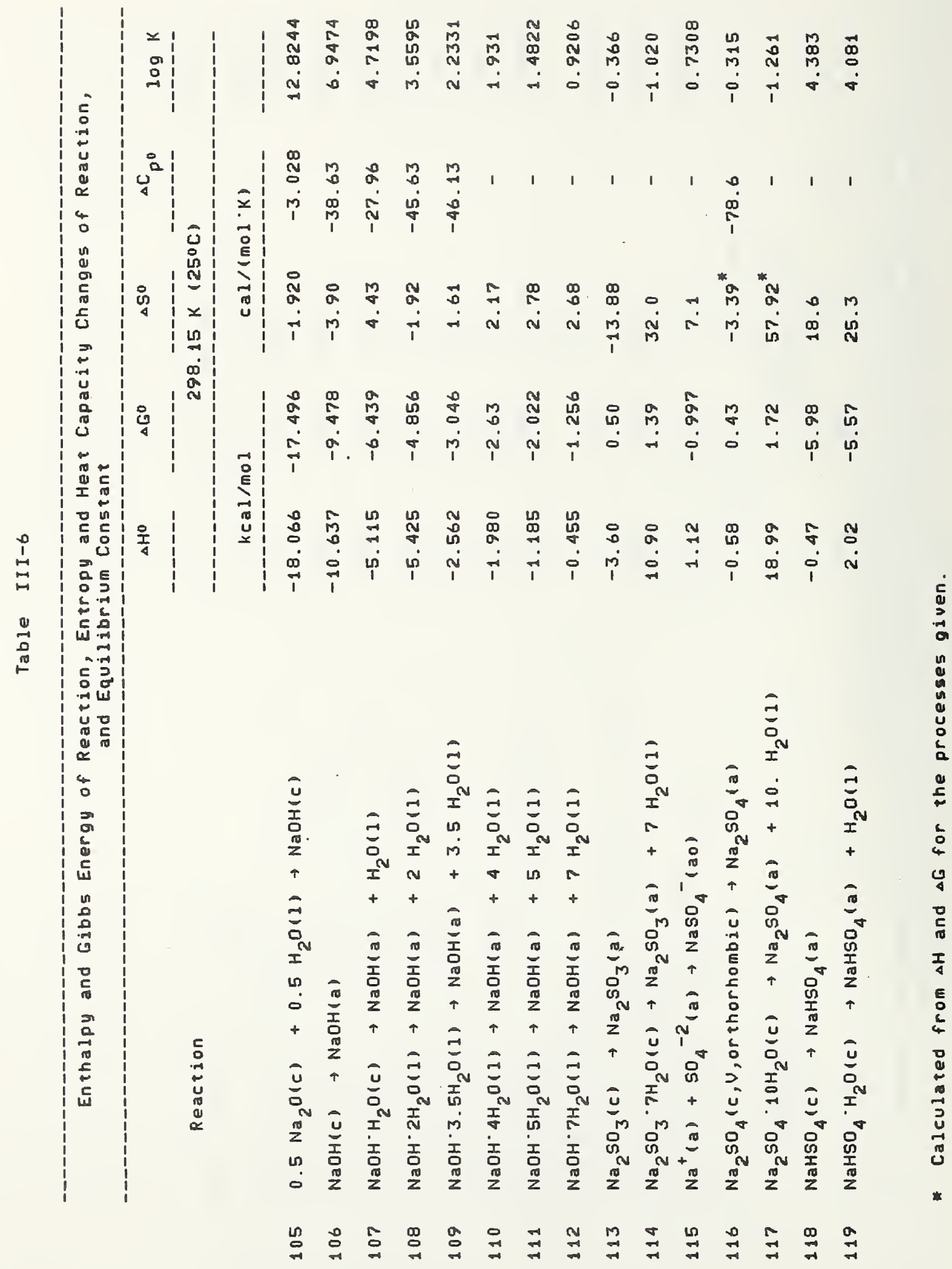




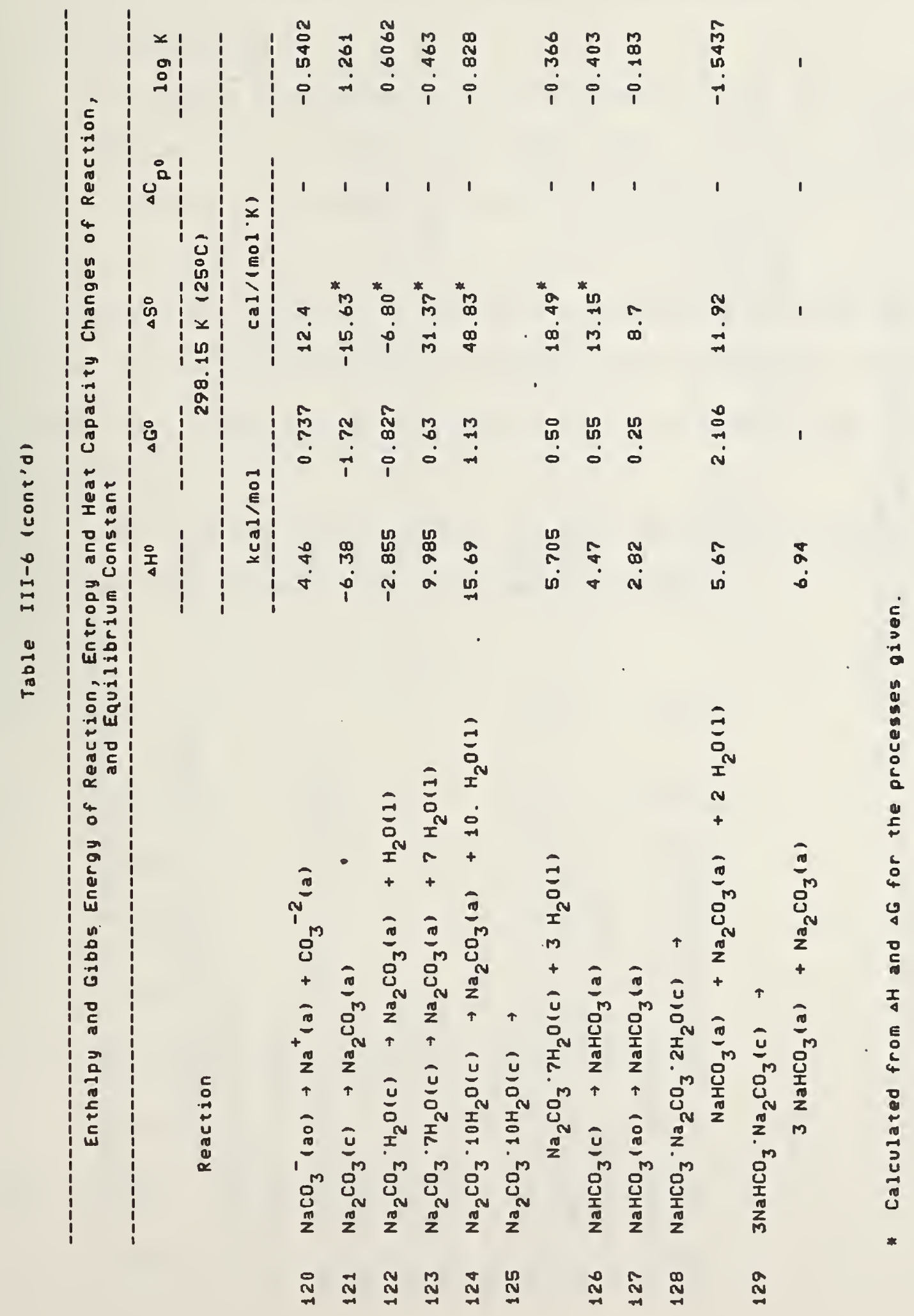




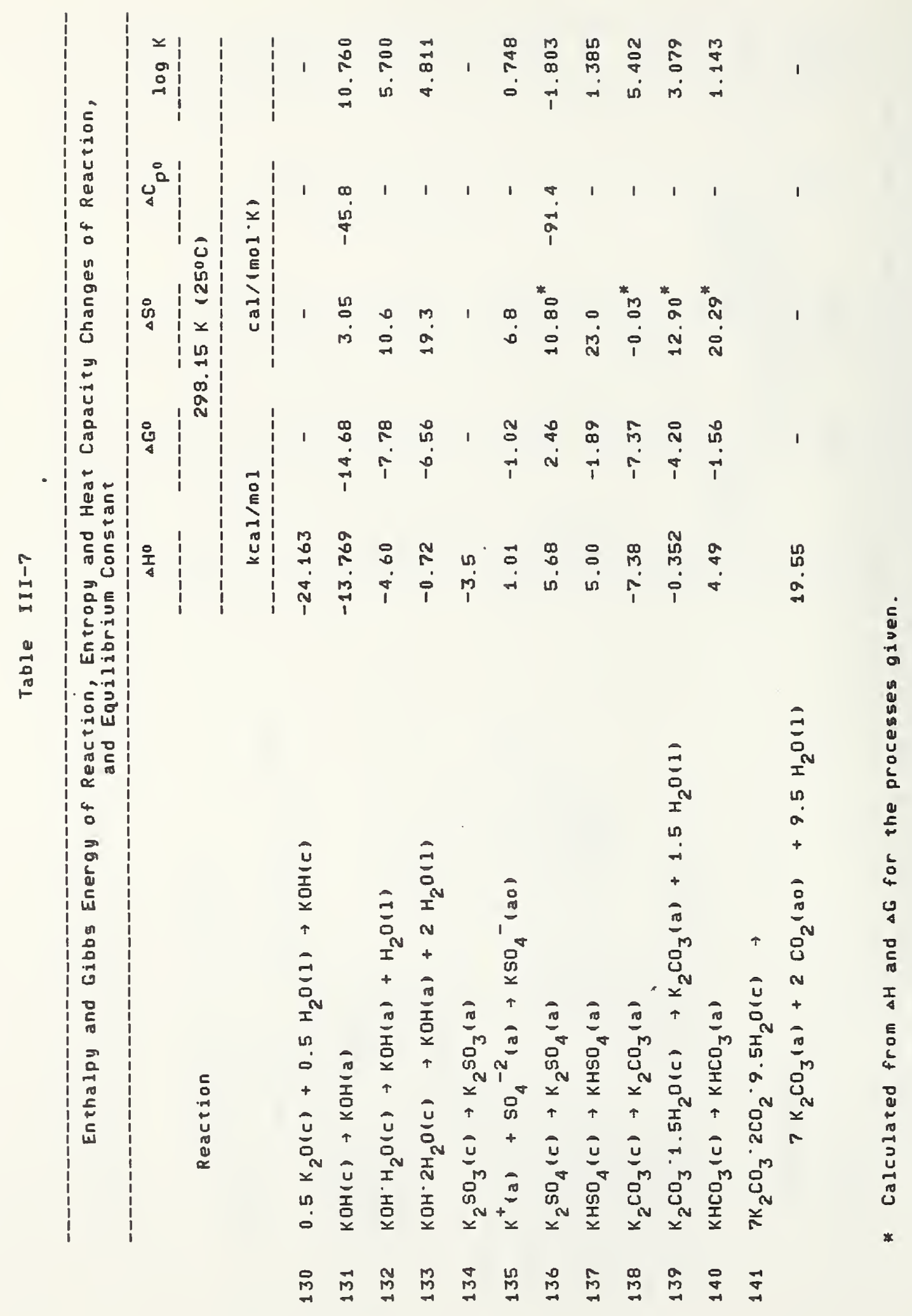


IV Tables for the Property Values of Binary Aqueous Systems as a Function of Concentration.

The values tabulated are $\phi_{L}$, the relative apparent molar enthalpy, $\gamma_{ \pm}$, the mean ionic activity coefficient, and $\phi$, the osmotic coefficient, as a function of the number of moles of $\mathrm{H}_{2} \mathrm{O}, \mathrm{nH}_{2} \mathrm{O}$, per mole of solute, and as a function of the number of moles of solute, mol $/ \mathrm{kg} \mathrm{H}_{2} \mathrm{O}$. Also included are the $\Delta_{f} H^{\prime} s$ as a function of the concentration obtained from NBS TN 270 [87NBS] and the parameters for the equations used to generate $\gamma_{ \pm}$and $\phi$ (for which see section VI). All values are reported at $298.15 \mathrm{~K}$.

The following tabular summary indicates the substances covered, in the order presented, and the properties tabulated.

\begin{tabular}{|c|c|c|c|c|c|c|c|c|}
\hline IV-I & $\mathrm{SO}_{2}$ & $\checkmark$ & & & IV -10 & $\mathrm{CaS}_{2} \mathrm{O}_{3}$ & $\checkmark$ & \\
\hline IV -2 & $\mathrm{H}_{2} \mathrm{SO}_{3}$ & $\checkmark$ & & & IV -11 & $\mathrm{Na}_{2} \mathrm{SO}_{3}$ & $\checkmark$ & $\checkmark$ \\
\hline IV -3 & $\mathrm{H}_{2} \mathrm{SO}_{4}$ & $\checkmark$ & $\checkmark$ & $\sqrt{ }$ & IV- 12 & $\mathrm{Na}_{2} \mathrm{SO}_{4}$ & $\checkmark$ & $\checkmark$ \\
\hline IV -4 & $\mathrm{FeSO}_{4}$ & $\checkmark$ & & & IV -13 & $\mathrm{NaHSO}_{4}$ & $\checkmark$ & $\checkmark$ \\
\hline IV -5 & $\mathrm{Fe}_{2}\left(\mathrm{SO}_{4}\right)_{3}$ & $\checkmark$ & & & IV -14 & $\mathrm{KOH}$ & $\checkmark$ & $\checkmark$ \\
\hline IV -6 & $\mathrm{Fe}\left(\mathrm{HSO}_{4}\right)_{3}$ & $\checkmark$ & & & IV -15 & $\mathrm{~K}_{2} \mathrm{SO}_{3}$ & $\checkmark$ & \\
\hline IV -7 & $\mathrm{MnSO}_{4}$ & $\checkmark$ & $\checkmark$ & $\checkmark$ & IV-16 & $\mathrm{K}_{2} \mathrm{SO}_{4}$ & $\checkmark$ & $\checkmark$ \\
\hline IV -8 & $\mathrm{MgSO}_{4}$ & $\checkmark$ & $\checkmark$ & $\checkmark$ & IV -17 & $\mathrm{KHSO}_{3}$ & $\checkmark$ & \\
\hline IV -9 & $\mathrm{CaSO}_{4}$ & $\checkmark$ & $\checkmark$ & $\checkmark$ & IV -18 & $\mathrm{KHSO}_{4}$ & $\checkmark$ & \\
\hline
\end{tabular}


Relative Apparent Molar Enthalpy and Activity Coefficients at $298.15 \mathrm{~K}$ Table IV-1.

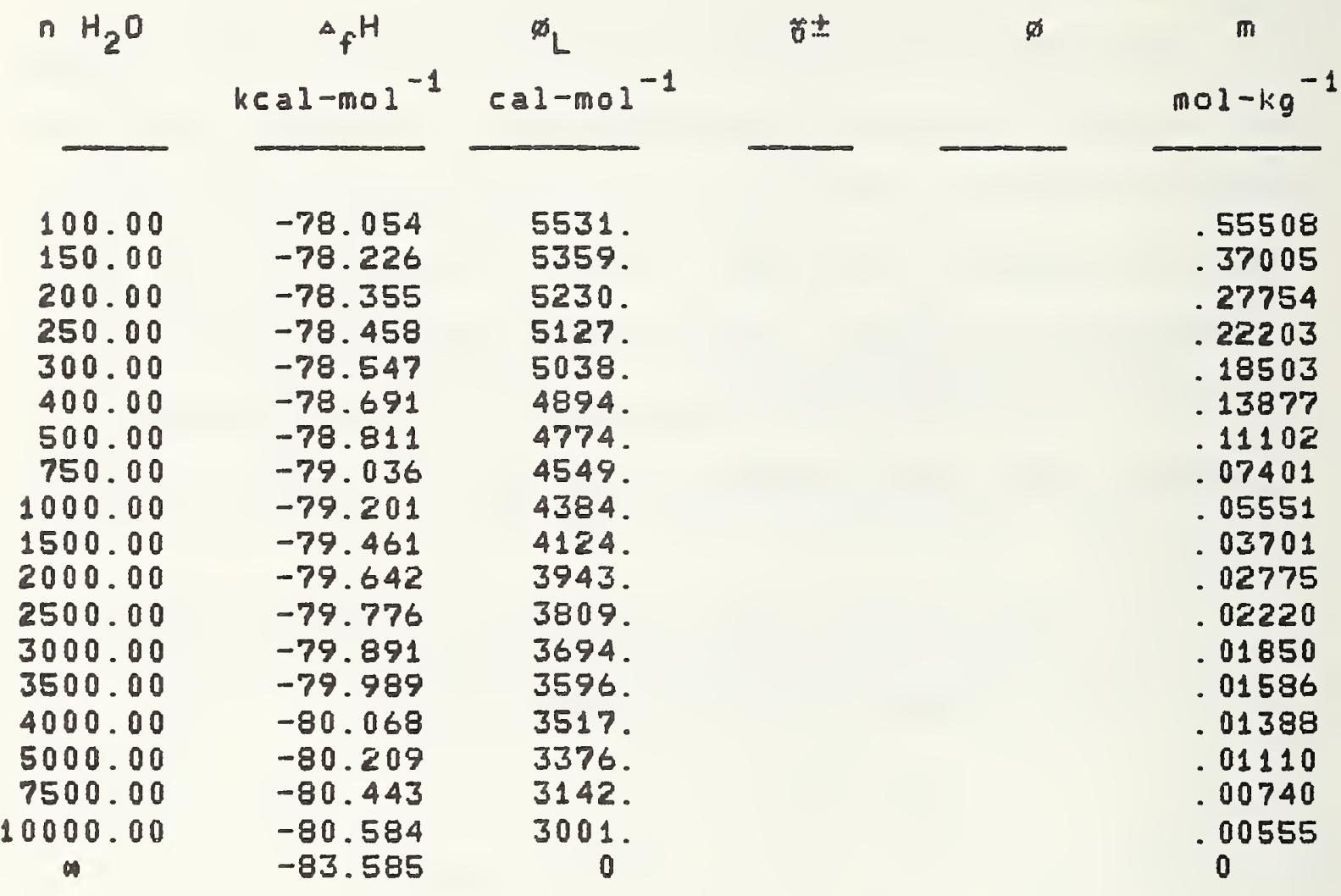

${ }_{f} H^{0}=\left[\begin{array}{l}-83.585 \mathrm{kcal}-\mathrm{mol} \\ {\left[\text { completely ionized }- \text { See note on Table IV-2. } \mathrm{H}_{2} \mathrm{SO}_{3}\right]}\end{array}\right.$

Enthalpy data taken from NBS TN 270-series [81NBS] 
Relative Apparent Molar Enthalpy and Activity Coefficients at $298.15 \mathrm{~K}$ Table IV-2
$\mathrm{H}_{2} \mathrm{SO}_{3}$

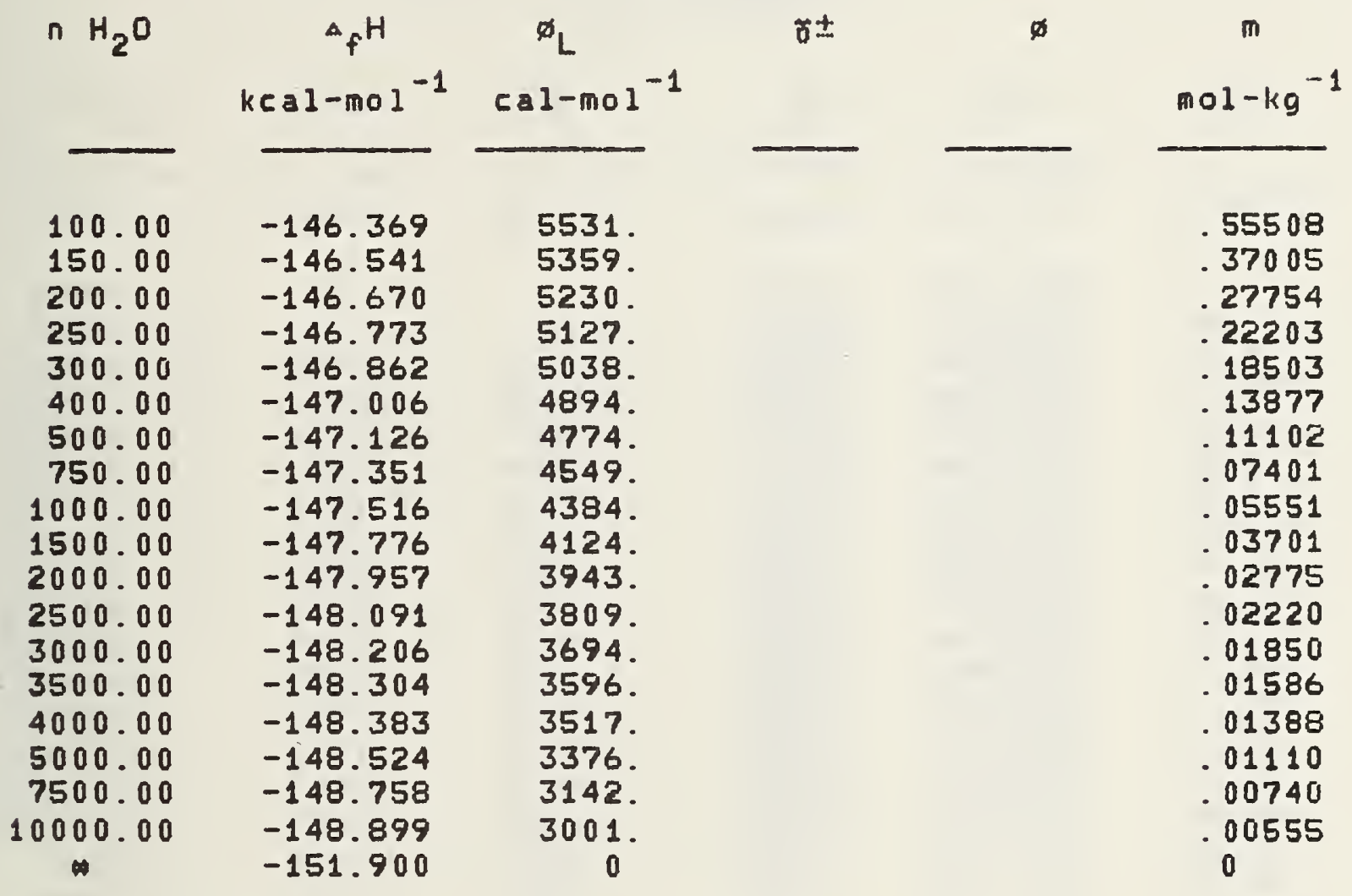
$\Delta_{f} H^{0}=-151.900 \mathrm{kcal}-\mathrm{mol}{ }^{-1} \quad$ Number of molalities $=18$ $\left[2 \mathrm{H}^{+}+\mathrm{SO}_{3}=\right.$ ]

Enthalpy data taken from NBS TN 270-Series [81NBS]

$\mathrm{H}_{2} \mathrm{SO}_{3}$ is thermodynamically equivalent to. $\mathrm{SO}_{2}+\mathrm{H}_{2} \mathrm{O}$. They are not distinct species. Both the $\mathrm{SO}_{2}$ and $\mathrm{H}_{2} \mathrm{SO}_{3}$ tables are for the formation of the real solution, including all effects of ionization. 
Table IV-3.

$$
\mathrm{H}_{2} \mathrm{SO}_{4}
$$

n
$\mathrm{H}_{2} \mathrm{O}$

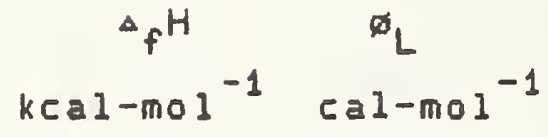
keal-mol

1.00

$-201.193$

1.50

2. 00

2. 50

3.00

3.50

4.00

4. 50

5.00

5.50

6. 00

7.00

8.00

9.00

10.00

12.00

15.00

20.00

25.00

30.00

40.00

50.00

75.00

100.00

115.00

150.00

200.00

300.00

400.00

500.00

600.00

700.00

800.00

900.00

1000.00

1500.00

2000.00

3000.00

4000.00
$-203.128$

$-204.455$

$-205.452$

$-206.241$

$-206.886$

$-207.428$

$-207.889$

$-208.288$

$-208.637$

$-208.944$

$-209.458$

$-209.865$

$-210.190$

$-210.451$

$-210.835$

$-211.191$

$-211.500$

$-211.660$

$-211.755$

$-211.869$

$-211.944$

$-212.068$

$-212.150$

$-212.192$

$-212.282$

$-212.387$

$-212.565$

$-212.709$

$-212.833$

$-212.950$

$-213.040$

$-213.128$

$-213.205$

$-213.275$

$-213.557$

$-213.785$

$-214.135$

$-214.415$
16127.

14192.

12865.

11868.

11079 .

10434.

9892.

9431.

9032.

8683.

8376.

7862.

7455.

7130 .

6869.

6485.

6129.

5820 .

5660 .

5565.

5451.

5376.

5252.

5170 .

5128.

5038.

4933.

4755.

4611.

4487.

4370 .

4280 .

4192.

4115.

4045 .

3763.

3535.

3185 .

2905. $\tilde{0} \pm$

$\not$

m

mol-kg ${ }^{-1}$

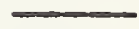

$-$

$\begin{array}{ll}- & 55.50807 \\ - & 37.00538\end{array}$

2. 4792

2. 3916

2. 3057

2. 2288

2. 1440

2. 0569

1.9726

1.8933

1.8196

1.6877

1. 5740

1. 4756

1. 3903

1. 2517

1. 1034

.9542

.8706

.8202

.7651

.7362

.7004

.6818

.6741

.6619

.6527

.6480

.6510

.6568

.6636

.6706

.6775

.6842

.6906

.7180

.7390

.7693

.7905
27.75403

22.20323

18.50269

15.85945

13.87702

12. 33513

11.10161

10.09238

9. 25134

7.92972

6.93851

6.16756

5.55081

4.62567

3.70054

2. 77540

2. 22032

1.85027

1.38770

1.11016

.74011

.55508

.48268

.37005

.27754

.18503

.13877

.11102

.09251

.07930

.06939

.06168

.05551

.03701

.02775

.01850

.01388

continued. . 
Relative Apparent Molar Enthalpy and ACtivity Coefficients at $298.15 \mathrm{~K}$

Table IV-3 continued.

$$
\mathrm{H}_{2} \mathrm{SO}_{4}
$$

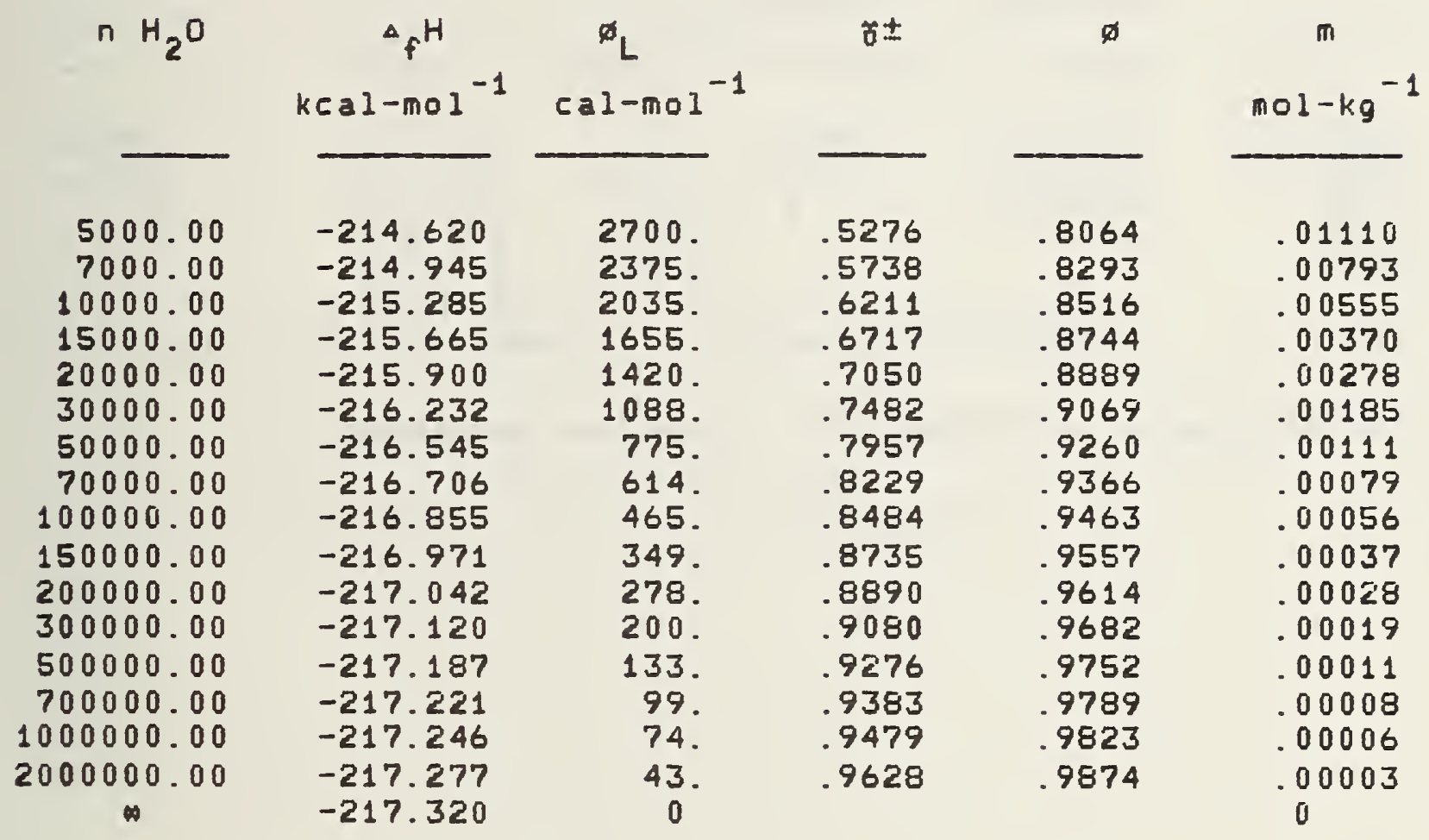

$\Delta_{f} H^{0}=\quad-217.320 \mathrm{keal}-\mathrm{mal}{ }^{-1} \quad$ Number of molalities $=55$

Enthalpy data taken from NBS TN 270-Series [81NBS]

Activity coefficient data from [815TA]

Constants for the activity coefficient equation below:
$B 1=-7.26977066 E+00$
$B 4=9.96540329 E+00$
$B 7=-1.92835211 E-01$
$B 2=1.27851225 E+01$
$B 5=-4.32909954 E+00$
$B 8=1.76565010 E-02$
$B 3=-1.42296622 E+01$
$B 6=1.17197320 E+00$
$B 9=-6.89852000 E-04$

Equation for the activity coefficient of $\mathrm{H}_{2} \mathrm{SO}_{4}$ :

$$
\ln \tilde{z}=\sum_{j=1}^{9} B m_{j}^{j / 2}
$$


Relative Apparent Molar Enthalpy and Activity Coefficients at $298.15 \mathrm{~K}$

Table IV-4.

$\mathrm{FeSO}_{4}$

ก $\mathrm{H}_{2} \mathrm{O}$

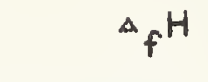

$\phi_{L}$

角立

$\varnothing$

m

$$
k \in a 1-m_{01}^{-1}
$$

cal-mol-1

$\operatorname{mol}-\mathrm{kg}^{-1}$
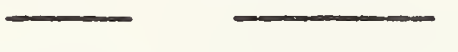

200.00

$-237.100$

40000.00

$-237.800$

$-238.600$

1500.

800 .

.27754

.00139

0

${ }_{f} H^{0}=-238.600 \mathrm{kcal}-\mathrm{mol} \mathrm{I}^{-1}$

Number of molalities =

2

Enthalpy data taken from NBS TN 270-Series [81NBS] 
Relative Apparent Molar Enthalpy and Activity Coefficients at $298.15 \mathrm{~K}$

$$
\begin{gathered}
\text { Table IU-5 } \\
\text { Fe, } \\
\left(50_{4}\right)_{3}
\end{gathered}
$$

$$
\begin{aligned}
& \text { ก } \mathrm{H}_{2} \mathrm{O} \\
& \Delta_{f} H \quad \phi_{L} \quad \text { 苦士 } \\
& \text { kcal-mol-1 cal-mol }{ }^{-1} \\
& 400.00 \\
& -653.000 \\
& -653.200 \\
& 22200 . \\
& -653.400 \\
& 22000 \text {. } \\
& -653.600 \\
& 21800 \text {. } \\
& -675.200 \\
& 0 \\
& \varnothing \\
& \text { mol-kg-1 } \\
& .13877 \\
& .05551 \\
& .02775 \\
& .01850 \\
& 0 \\
& { }_{f} H^{0}=
\end{aligned}
$$

Enthalpy data taken from NBS TN 270-Series [81NBS]

45 
Relative Apparent Molar Enthalpy and Activity Coefficients at $298.15 \mathrm{~K}$

$$
\begin{gathered}
\text { Table IU-6. } \\
\text { FelHSO } \\
4
\end{gathered}
$$
ก $\mathrm{H}_{2} \mathrm{O}$
${ }_{f} \mathrm{H}$
$\infty_{L}$
莸
$\emptyset$
m

$$
k \in a 1-\operatorname{mol} 1^{-1}
$$
cal-mol ${ }^{-1}$

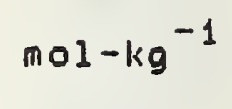
600.00
4
$-646.500$
1300 .
-15760 .
.09251

${ }_{f} H^{0}=-647.800 \mathrm{kcal}-\mathrm{mol}^{-1}$

Number of molalities =

1

Enthalpy data taken from NBS TN 270-Series [81NBS] 
Relative Apparent Molar Enthalpy and Activity Coefficients at $298.15 \mathrm{~K}$

$$
\begin{gathered}
\text { Table IV } \\
\text { Mnso } \\
4
\end{gathered}
$$

n $\mathrm{H}_{2} \mathrm{O}$

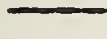

20.00

30.00

50.00

100.00

200.00

400.00

1000.00

2000.00

3000.00

5000.00

10000.00

20000.00

50000.00

100000.00

4

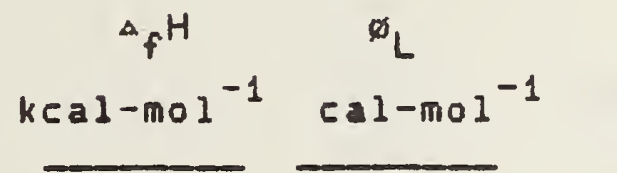

2520.

2080 .

1920.

1770.

1610 .

1460 .

1250 .

1030 .

960 .

840 .

700 .

570.

420.

330 .

0
苦士

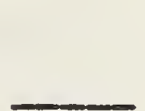

.0439

.0428

.0510

.0734

.1072

.1540

.2399

.3236

.3784

.4509

.5499

.6427

.7473

.8103 $\varnothing$

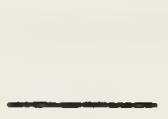

.7307

.5562

.4823

.4846

.5168

.5572

.6248

.6842

.7198

.7631

.8163

.8610

.9065

.9317 m mol $-\mathrm{kg}^{-1}$

2.77540

1.85027

1.11016

.55508

.27754

.13877

.05551

.02775

.01850

.01110

.00555

.00278

.00111

.00056

$\Delta_{f} H^{0}=-270.100 \mathrm{keal}-m o l^{-1} \quad$ Number of molalities $=14$

Enthalpy data taken from NBS TN 270-Series [81NBS]

ACtivity coefficient data from [74PIT/MAY]

Constants for the activity coefficient equation (eqns. VI-1 thru 7):

$b=1.20000000 E+00$

$\beta^{0}=2.01000000 E-01$

$C^{\varnothing}=1.82000000 E-02$ $a_{1}=1.40000000 E+00$

$\beta^{1}=2.98000000 E+00$

$a_{2}=1.20000000 E+01$

$\beta^{3}=0.00000000 E+00$ 
Relative Apparent Molar Enthalpy and Activity Coefficients at $298.15 \mathrm{~K}$

Table IU-8. $\mathrm{MgSO}_{4}$

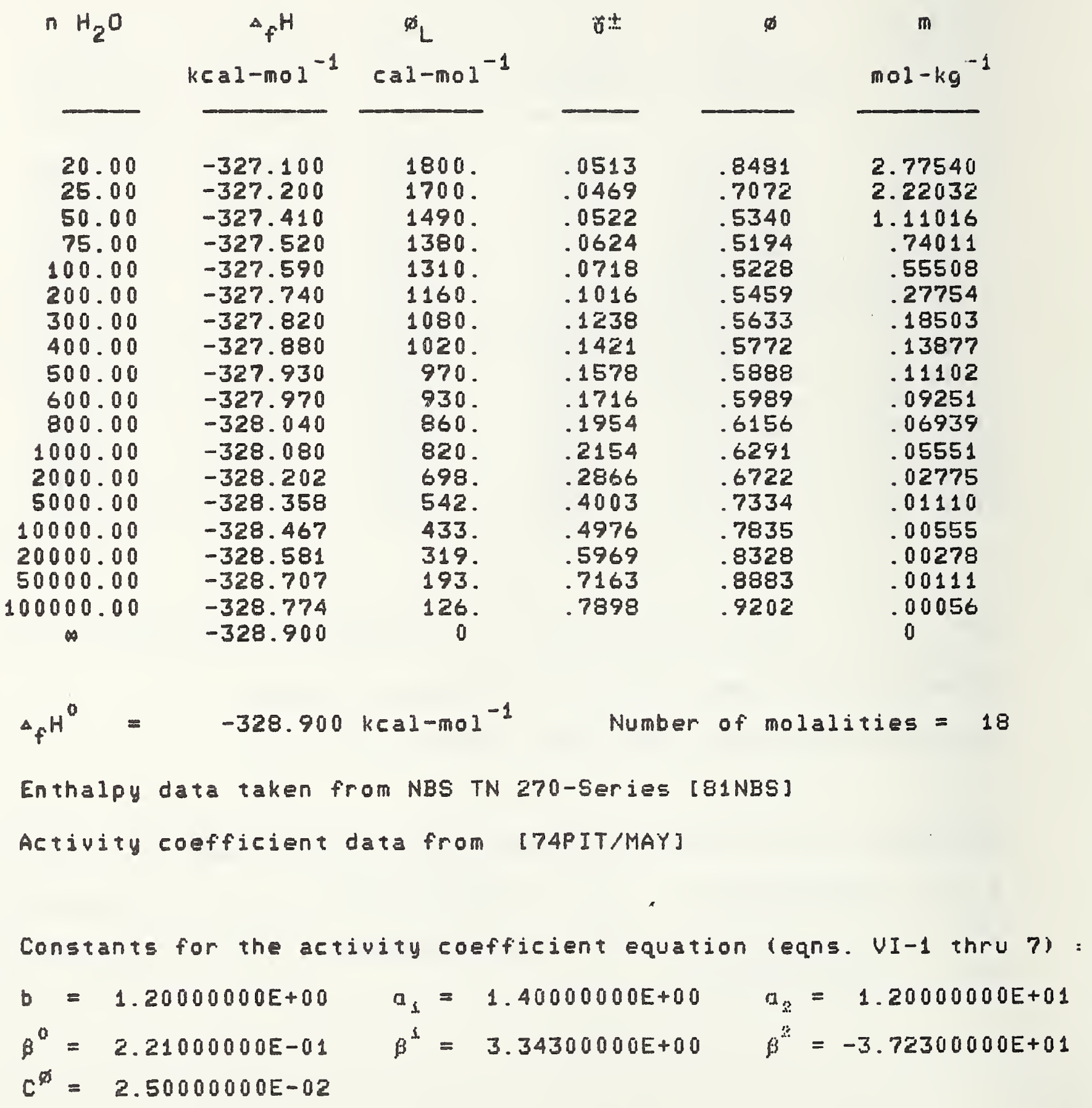


Relative Apparent Molar Enthalpy and Activity Coefficients at $278.15 \mathrm{~K}$ Table IV-9.
Caso
4

n $\mathrm{H}_{2} \mathrm{O}$

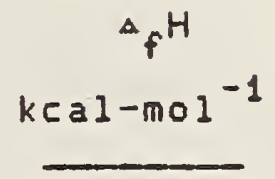

$-346.414$

5000.00

7500.00

10000.00

20000.00

50000.00

100000.00

500000.00

1000000.00

4
$-346.518$

$-346.566$

$-346.685$

$-346.827$

$-346.915$

$-347.009$

$-347.026$

$-347.060$ $\phi$

cal-mol-1

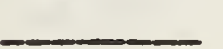

${ }_{f} H^{0}$
ช⿱艹口犬士

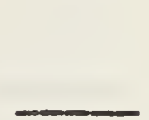

646.

542 .

494.

375 .

233.

145.

51.

34.

0

.3714

.4276

.4695

.5729

.7000

.7791

.8986

.9284 $\varnothing$

m

mol-kg $g^{-1}$
.01110

.00740

.00555

.00278

.00111

.00056

.00011

.00006

Enthalpy data taken from NBS TN 270-Series [81NBS]

Activity coefficient data from [74PIT/MAY]

Constants for the activity coefficient equation (eqns. VI-1 thru 7 ) :
$b=1.20000000 E+00$
$a_{1}=1.40000000 E+00$
$a_{2}=1.20000000 E+01$
$B^{0}=2.00000000 E-01$
$\beta^{2}=2.65000000 E+00$
$\beta^{2}=-5.57000000 E+01$
$c^{\varnothing}=0.00000000 E+00$ 
Relative Apparent Molar Enthalpy and Activity Coefficients at $298.15 \mathrm{~K}$

Table IU-10.

$\operatorname{cas}_{2}{ }_{3}$

n $\mathrm{H}_{2} \mathrm{O}$

$\Delta_{f} H$

$\phi_{L}$

茫

$\varnothing$

m

$$
\text { keal-mol }{ }^{-1} \text { cal-mol }{ }^{-1}
$$

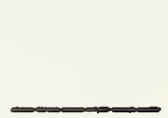

$\operatorname{mol}-\mathrm{kg}^{-1}$

$35.00 \quad-284.560$

100.00

1040 .

800 .

650 .

580 .

500 .

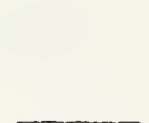

$\underline{n}$

200.00

400.00

$-284.800$

$-284.950$

1000.00

$-285.020$

$-285.100$

$-285.600$

0

1.58594

.55508

.27754

.13877

.05551

0

$\Delta_{f} H^{B}=-285.600 \mathrm{kCal}-\mathrm{mol}^{-1}$

Number of molalities $=$

5

Enthalpy data taken from NBS TN 270-Series [81NBS] 
Relative Apparent Molar Enthalpy and Activity Coefficients at $298.15 \mathrm{~K}$ Table IV-11.

$\mathrm{Na}_{2} \mathrm{SO}_{3}$
ก $\mathrm{H}_{2} \mathrm{O}$
$\phi_{L}$
$r \pm$
$\emptyset$
m kcal-mol ${ }^{-1}$ cal-mol ${ }^{-1}$
$\operatorname{mol}-\mathrm{kg}^{-1}$
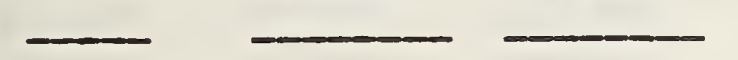
30.00
50.00
100.00
200.00
800.00
2000.00
10000.00
50000.00
$\rightarrow$

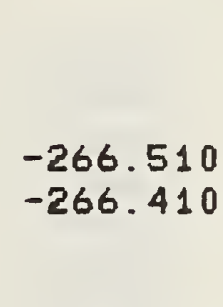
$-266.700$

$\begin{array}{cc} & .1989 \\ & .2292 \\ & .2866 \\ 190 . & .3529 \\ 290 . & .5029 \\ & .6074 \\ & .7705 \\ & .8812 \\ 0 & \end{array}$
.7124
1.85027
1.11016
.55508
.27754
.06939
.02775
.00555
.00111
9593
0

$\Delta_{f} H^{0}=\quad-266.700 \mathrm{kcal-mol}-1 \quad$ Number of molalities $=8$

Enthalpy data taken from NBS TN 270-Series [81NBS]

Activity coefficient data from [81GOL]

Constants for the activity coefficient equation (eqns. UI-8,7):
$B=1.32416014 E+00$
$E=0.00000000 E+00$
$H=0.00000000 E+00$
$C=-2.69200069 E-01$
$F=0.00000000 E+00$
$I=0.00000000 E+00$
$D=6.67319796 E-02$
$G=0.00000000 E+00$
$J=0.00000000 E+00$ 
Relative Apparent Molar Enthalpy and Activity Coefficients at $298.15 \mathrm{~K}$

Table IV-12.

$\mathrm{Na}_{2} \mathrm{SO}_{4}$

ก $\mathrm{H}_{2} \mathrm{O}$

18.00
20.00

25.00

28.37

30.00

40.00

50.00

60.00

80.00

100.00

120.00

140.00

160.00

180.00

200.00

250.00

300.00

350.00

400.00

500.00

600.00

800.00

1000.00

2000.00

3000.00

4000.00

5000.00

10000.00

20000.00

50000.00

$\infty$
$\operatorname{keal}_{f^{H}}^{H} \operatorname{mol}^{-1}$

$-333.852$

$-333.800$

$-333.593$

$-333.491$

$-333.432$

$-333.165$

$-332.988$

$-332.840$

$-332.605$

$-332.420$

$-332.275$

$-332.184$

$-332.122$

$-332.076$

$-332.040$

$-331.980$

$-331.943$

$-331.918$

$-331.901$

$-331.879$

$-331.867$

$-331.857$

$-331.855$

$-331.872$

$-331.892$

$-331.907$

$-331.920$

$-331.958$

$-331.992$

$-332.027$

$-332.100$
${ }^{\infty} L$

$c a 1-m o 1^{-i}$

$-1752$.

-1700 .

$-1493$.

$-1391$.

-1332 .

-1065 .

-888 .

-740 .

-505 .

-320 .

-175 .

$-84$.

$-22$.

24.

60.

120.

157.

182.

199.

221.

233.

243.

245.

228.

208.

193.

180.

142.

108.

73.

0

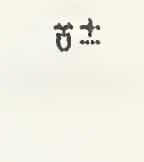

.1389

.1411

.1494

.1558

.1590

.1780

.1953

.2107

.2367

.2581

.2762

.2919

.3058

.3183

.3296

.3540

.3743

.3919

.4072

.4333

.4549

.4894

.5163

.5991

.6457

.6774

.7010

.7677

.8235

$.880 \hat{5}$ $\varpi$

m

$\operatorname{mol}-\mathrm{kg}^{-1}$

.6735

.6539

.6303

.6252

.6244

.6302

.6417

.6532

.6726

.6876

.6996

.7073

.7175

.7246

.7308

.7435

.7537

.7622

.7694

.7813

.7910

.8060

.8176

.8520

.8707

.8832

.8924

.9178

.9385

.9589
3.08378

2. 77540

2. 22032

1.95658

1. 85027

1. 38770

1. 11016

.92513

.69385

.55508

.46257

.39649

.34693

.30838

.27754

.22203

.18503

.15859

.13877

.11102

.09251

.06939

.05551

.02775

.01850

.01388

.01110

.00555

.00278

.00111

a

$\Delta_{f} H^{0}=\quad-332.100 \mathrm{keal}-\mathrm{mol}^{-1}$

Number of molalities $=30$

Enthalpy data taken from NBS TN 270-Series [81NBS]

Activity coefficient data from [81GOL]

Constants for the activity coefficient equation ieqns. UI-8,9):

$B=1.21597315 E+00$
$C=-3.5572852 E-01$
$D=8.29465562 E-02$$$
E=
$$

$E=-4.86954126 E-03$

$F=0.00000000 E+00$

$H=0.00000000 E+00$

$E=0.00000000 E+00$

$I=0.00000000 E+00$

$J=0.00000000 E+00$ 
Relative Apparent Molar Enthalpy and Activity Coefficients at $298.15 \mathrm{~K}$

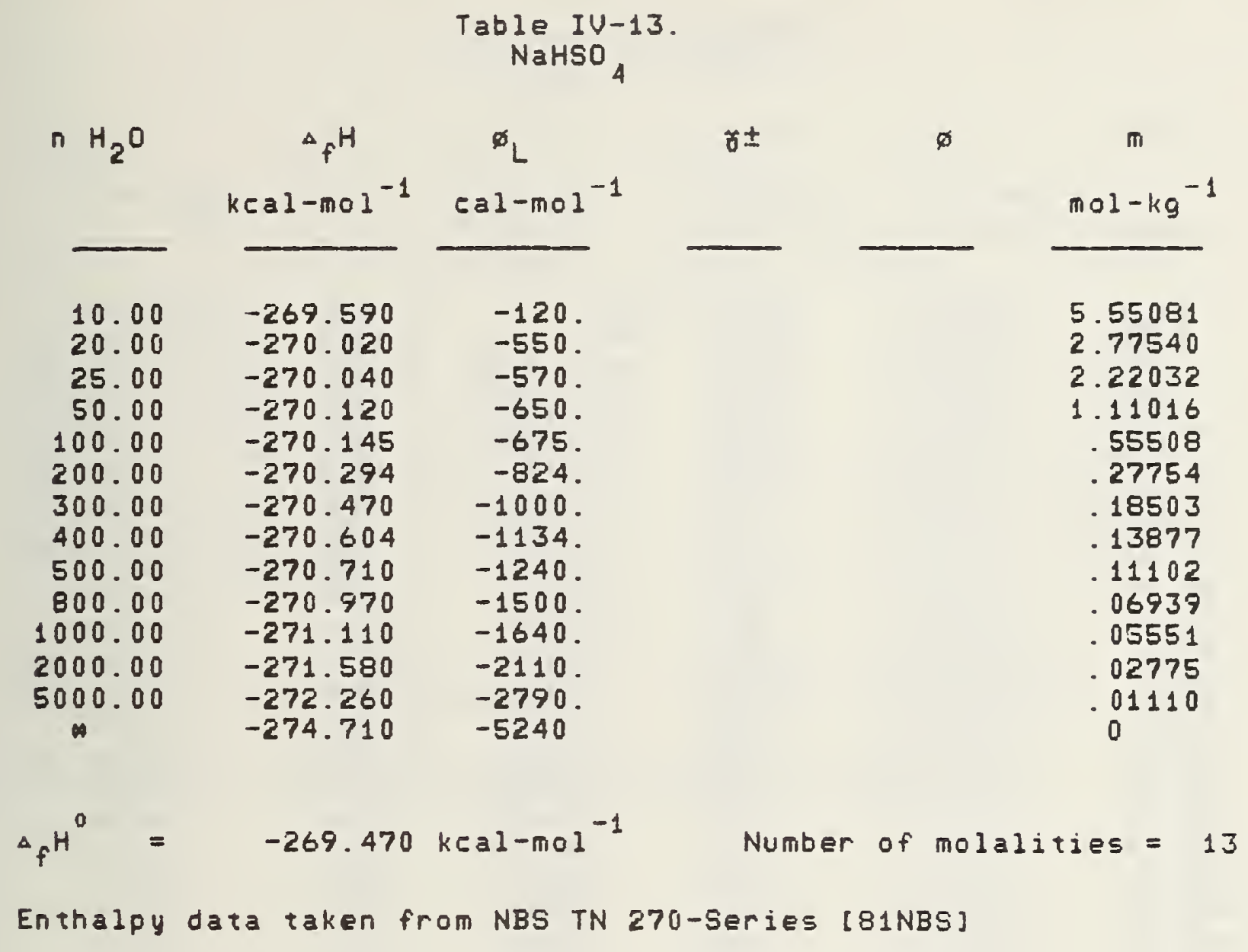


Relative Apparent Molar Enthalpy and Activity Coefficients at $298.15 \mathrm{~K}$ Table IV-14. $\mathrm{KOH}$

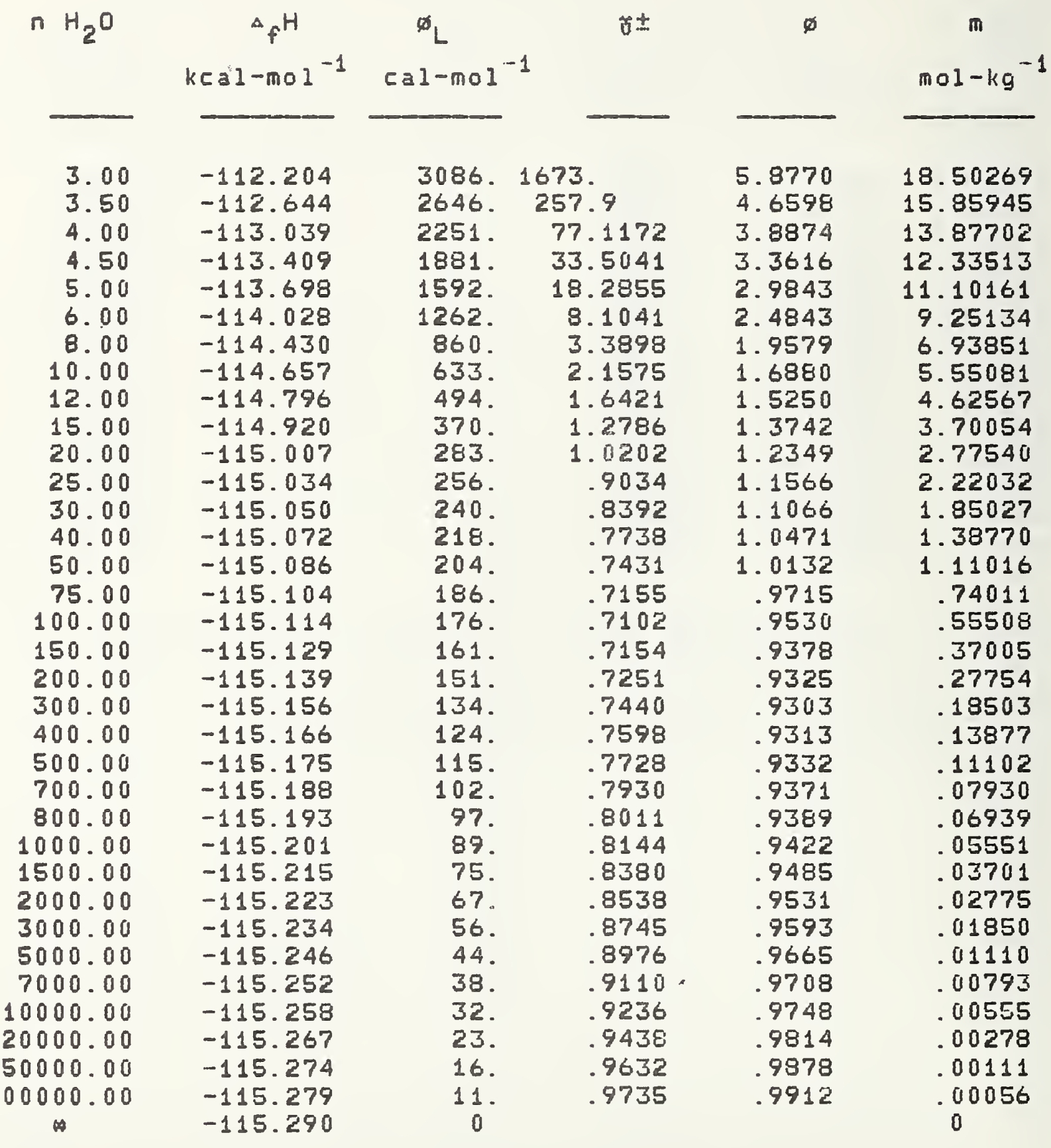

$\Delta_{f} H^{0}=-115.290 \mathrm{keal-mol}{ }^{-1} \quad$ Number of molalities $=34$

Enthalpy data taken from NBS TN 270-Series [81NBS]

Activity coefficient data from [72HAM/WU]

Constants for the actiuity coefficient equation (eqns. UI-8,9):
$B=1.15000000 E+00$
$E=2.99336000 E-04$
$H=0.00000000 E+00$
$C=2.30258509 E-01$
$F=3.91439000 E-07$
$I=0.00000000 E+00$
$D=6.04889000 E-03$
$G=0.00000000 E+00$
$\mathrm{J}=0.00000000 \mathrm{E}+00$ 
Relative Apparent Molar Enthalpy and Activity Coefficients at $298.15 \mathrm{~K}$

$$
\text { Table IV-15. }
$$

ก $\mathrm{H}_{2} \mathrm{O}$
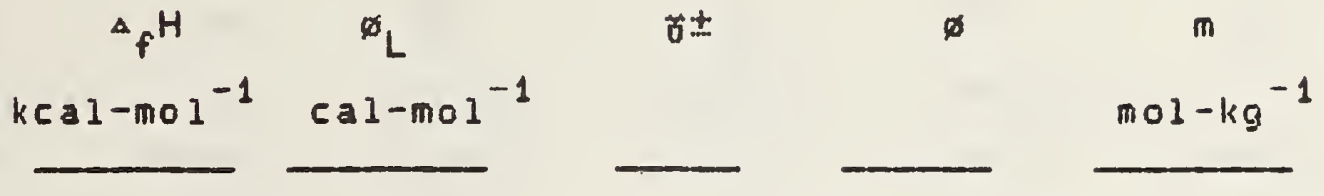

$50.00-272.200$

300

1.11016

$-271.500$

1000 .

.55508

$200.00-271.200$

1300 .

.27754

$350.00 \quad-271.200$

1300 .

.15859

1200 .

1100 .

.13877

$500.00-271.400$

1000 .

$-271.500$

600.00

$-271.900$

600 .

.11102

.09251

.05551

$-272.500$

0 0

${ }_{{ }_{f}} H^{0}=-272.500 \mathrm{kcal}-\mathrm{mol}^{-1} \quad$ Number of molalities $=8$

Enthalpy data taken from NBS TN 270-Series [81NBS] 
Relative Apparent Molar Enthalpy and Activity Coefficients at $298.15 \mathrm{~K}$

Table IV-16.

$$
\mathrm{K}_{2} \mathrm{SO}_{4}
$$

ก $\mathrm{H}_{2} \mathrm{O}$

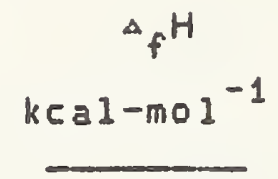

50.00

60.00

80.20

100.00

200.00

300.00

400.00

500.00

600.00

700.00

800.00

1000.00

1500.00

2000.00

3000.00

5000.00

8000.00

10000.00

20000.00

50000.00

100000.00

500000.00

$\infty$

-338.460
-338.360
-338.180
-338.050
-337.820
-337.770
-337.730
-337.720
-337.708
-337.706
-337.707
-337.711
-337.720
-337.732
-337.751
-337.780
-337.806
-337.819
-337.854
-337.889
-337.909
-337.938
-337.960

$\sigma_{L}$

$$
\text { cal-mol }-1
$$

-500 .

-400 .

-220 .

-90 .

140 .

190.

230 .

240.

252.

254.

253.

249.

240 .

228.

209.

180.

154.

141.

106 .

71.

51.

22.

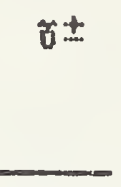

-

.2422

.3079

.3515

.3846

.4112

.4335

.4526

.4694

.4976

.5490

.5851

.6344

.6927

.7413

.7625

.8205

.8791

.9117

.9587

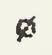

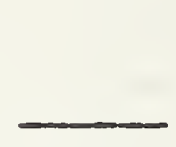

-

.6743

.6832

.7139

.7345

.7501

.7627

.7731

.7821

.7898

.8028

.8260

.8418

.8629

.8870

.9064

.9147

.9367

.9582

.9697

.9860 m

$\operatorname{mol}-k g^{-1}$

1.11016

.92513

.69212

.55508

.27754

.18503

.13877

.11102

.07251

.07930

.06939

.05551

.03701

.02775

.01850

.01110

.00694

.00555

.00278

.00111

.00056

.00011

$\Delta_{f} H^{0}=-337.960 \mathrm{kcal}-\mathrm{mol}^{-1}$

Number of molalities $=22$

Enthalpy data taken from NBS TN 270-Series [81NBS]

Activity coefficient data from [8160L]

Constants for the activity coefficient equation (eqns. UI-8,9):
$B=9.43830073 E-01$
$E$
$0.00000000 E+00$
$H=0.00000000 E+00$
$C=-8.85985775 E-02$
$F=0.00000000 E+00$
$I=0.00000000 E+00$
$D=0.00000000 E+00$
$G=0.00000000 E+00$
$J=$
$0.00000000 E+00$ 
Relative Apparent Molar Enthalpy and Activity Coefficients at $298.15 \mathrm{~K}$

$$
\text { Table IU-17. }
$$

$$
\mathrm{KHSO}_{3}
$$
ก $\mathrm{H}_{2} \mathrm{O}$
${ }_{f} H$
$\phi_{L}$
苫京
$\varnothing$
m

$$
k \in a 1-\operatorname{mol}^{-1}
$$
cal-mol $1^{-1}$
$\operatorname{mol}-k g^{-1}$
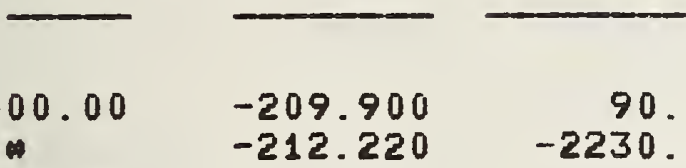
$\begin{array}{rrr}400.00 & -209.900 & 90 . \\ & -212.220 & -2230 .\end{array}$
$0_{0}^{13877}$

${ }_{f} H^{0}=-209.990 \mathrm{keal}-\mathrm{mol}^{-1}$

Number of molalities =

1

Enthalpy data taken from NBS TN 270-Series [81NBS] 
Relative Apparent Molar Enthalpy and Activity Coefficients at $298.15 \mathrm{~K}$

Table IU-18.

$\mathrm{KHSO}_{4}$
ก $\mathrm{H}_{2} \mathrm{O}$
${ }_{f} \mathrm{H}$
$\phi_{L}$
䒘立
$\varnothing$
m

$$
k \in a_{1-m o 1}-1
$$
$\operatorname{cal} 1-\operatorname{mol} 1^{-1}$
$\operatorname{mol}-\mathrm{kg}^{-1}$
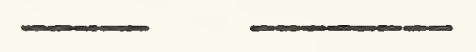
20.00
$-273.500$

$$
\begin{aligned}
& -1100 \\
& -1100 \\
& -1200 \\
& -1400 \\
& -1440 \\
& -1440 \\
& -1780 \\
& -2160 \\
& -2280 \\
& -2650 \\
& -3300
\end{aligned}
$$$$
-1100 \text {. }
$$
50.00
$-273.500$
$-275.700$
$-277.640-5240$.
2.77540
2.22032
1. 11016
.55508
.27754
.25231
.13877
.06939
.05551
.02775
.01110
.00617
0

$\Delta_{f} H^{0}=-272.400 \mathrm{kcal}-\mathrm{mol}^{-1}$

Number of molalities $=12$

Enthalpy data taken from NBS TN 270-Series [81NBS] 
V Documentation for the Thermochemical Values for Some Processes

The arrangement is as follows:

$\mathrm{V}-1$ Processes involving $\mathrm{Fe}$

V-2 Processes involving Mg

V-3 Processes involving $\mathrm{Ca}$

$\mathrm{V}-4$ Processes involving $\mathrm{Na}$ 
$\mathrm{V}-1$. Processes involving $\mathrm{Fe}$

The Dissociation of $\mathrm{FeOH}^{+}$(ao) (reaction 28)

[63BOL/ARV] determined the hydrolysis $\mathrm{K}$ of $\mathrm{Fe}^{+2}(\mathrm{a})$ to $\mathrm{FeOH}^{+}(\mathrm{ao})$ as a function of $T$.

The Solution Process for $\mathrm{Fe}(\mathrm{OH})_{2}$ (c) (reactions $29,35,36$ )

[83TH0] measured the $\Delta H$ for the precipitation of $\mathrm{Fe}(\mathrm{OH})_{2}$ from $\mathrm{FeSO}_{4}(\mathrm{aq})$ with $\mathrm{KOH}(\mathrm{aq})$. [53LEU/KOL] and [63DAU/DAU] measured the $\mathrm{K}$ 's for the formation of $\mathrm{FeOH}^{+}(\mathrm{aO})$. [56GAY/WO0] obtained the K's for the formation of $\mathrm{Fe}(\mathrm{OH})_{3}^{-1}(\mathrm{aO})$ and $\mathrm{Fe}(\mathrm{OH})_{4}^{-2}(\mathrm{aO})$ from $\mathrm{Fe}(\mathrm{OH})_{2}(\mathrm{c})$.

The Solution Process for FeSO $4 \mathrm{nH}_{2} \mathrm{O}(\mathrm{c}) \quad(n=7,4,1,0)$ (reactions 42-45)

$[63 \mathrm{ADA} / \mathrm{KEL}]$ measured the $\triangle \mathrm{H}$ soln in $\mathrm{HCl}(\mathrm{aq})$ of $\mathrm{FeSO}_{4} \cdot \mathrm{H}_{2} \mathrm{O}(\mathrm{c})$ and $\mathrm{FeSO}_{4} \cdot 7 \mathrm{H}_{2} \mathrm{O}(\mathrm{c})$. [14FOR] measured the $\Delta \mathrm{H}(\mathrm{soln})$ of all the hydrates, as well as the anhydrous salt, in $\mathrm{H}_{2} \mathrm{O} \cdot \mathrm{H}_{2} \mathrm{O}$ vapor pressure measurements of the hydrates have been made by [64KOH/ZAS] and [14BIL].

The Solution Process for $\mathrm{FeCO}_{3}$ (c) (reaction 52)

[35KEL/AND] cite the $K$ to be $3.14 \times 10^{-11}$. [(18)75BER] measured the $\Delta \mathrm{H}$ for the precipitation from $\mathrm{FeSO}_{4}(\mathrm{aq})$ and $\mathrm{K}_{2} \mathrm{CO}_{3}(\mathrm{aq})$. 


\section{$\mathrm{V}-2$. Processes involving $\mathrm{Mg}$}

The Formation Properties of $\mathrm{MgO}$ (c,macro) and $\mathrm{MgO}$ (c,micro) (reactions 68,69)

$\Delta_{f} H^{\circ}$ 's of $\mathrm{MgO}$ (Macro-crystal) and $\mathrm{MgO}$ (micro-crystal) are obtained primarily from the measurements of [43SHO/HUF] on the $\Delta \mathrm{H}^{\prime} \mathrm{s}$ of solution of $\mathrm{Mg}(\mathrm{c})$ and $\mathrm{MgO}$ in aqueous $\mathrm{HCl}$, the direct combustion measurements on $\mathrm{Mg}(\mathrm{cs})$ by [51 HOL/HUB], and the measurements of $[49 \mathrm{GIA}]$ on $\Delta \mathrm{H}$ (trans) $\mathrm{MgO}$ (macro) $\rightarrow \mathrm{MgO}$ (micro).

The Relationship between $\mathrm{MgO}$ (macro and micro) and $\mathrm{Mg}(\mathrm{OH})_{2}$ (c) (reactions 68, 69)

The $\Delta H$ measurements of $[37 \mathrm{WEL} / T A Y]$ and $[38 T A Y / W E L]$ on the solution of $\mathrm{MgO}$ (c,macro) and $\mathrm{Mg}(\mathrm{OH})_{2}(\mathrm{c})$ in $2 \mathrm{~N} \mathrm{HCl}$, as well as the measurements of [37GIA/ARC], (micro), [48TOR/SAH], and [43SHO/HUF] on the solution of $\mathrm{MgO}$ (c, micro) and $\mathrm{Mg}(\mathrm{OH})_{2}(\mathrm{c})$ in $1 \mathrm{~N} \mathrm{HCl}$, are used to determine the relationship. The decomposition measurements of $\mathrm{Mg}(\mathrm{OH})_{2}(\mathrm{c})$ by [37GIA/ARC] are in agreement.

The Dissociation of $\mathrm{MgOH}^{+}(\mathrm{aq})$ (reaction 72)

The $\log K=-2.58$ is obtained from the tabulation of [64SIL/MAR] and [63HOS2].

\section{The Precipitation of $\mathrm{Mg}(\mathrm{OH})_{2}$ (amorp) (reaction 71)}

[83THO] and [87BER] measured the $\triangle \mathrm{H}$ (pptn) of $\mathrm{Mg}(\mathrm{OH})_{2}$ from $\mathrm{MgSO}_{4}$ solutions with $\mathrm{KOH}(\mathrm{aq})$ and $\mathrm{NaOH}(\mathrm{aq})$, respectively. In addition, [87BER] measured the $\Delta \mathrm{H}$ from $\mathrm{MgCl}_{2}(\mathrm{aq})$ with $\mathrm{NaOH}(\mathrm{aq})$.

The Solution Process for $\mathrm{MgSO}_{3} \cdot \mathrm{nH}_{2} \mathrm{O}$ (c) $(\mathrm{n}-6,3,0)^{\prime}$ (reactions 73-77)

The $\triangle \mathrm{H}$ measurement of [87HAR] on the solution of $\mathrm{MgSO}_{3} \cdot 6 \mathrm{H}_{2} \mathrm{O}(\mathrm{c})$, $\mathrm{MgSO}_{3} \cdot 3 \mathrm{H}_{2} \mathrm{O}(\mathrm{c})$, and $\mathrm{MgSO}_{3}(\mathrm{c})$ in $\mathrm{HCl}(\mathrm{aq})$ and on the solution of a mixture of $\mathrm{MgSO}_{3} \cdot 6 \mathrm{H}_{2} \mathrm{O}(\mathrm{c})$ and $\mathrm{MgCl}_{2} \cdot 6 \mathrm{H}_{2} \mathrm{O}(\mathrm{c})$ in $\mathrm{H}_{2} \mathrm{SO}_{3}(\mathrm{aq})$ result in values for the $\Delta_{\mathrm{f}} \mathrm{H}^{\circ} \mathrm{s}$ for $\mathrm{MgSO}_{3}(\mathrm{c})$ and the two hydrates. The $\Delta \mathrm{H}$ soln is thus calculated from $\Sigma \Delta_{f} H^{\circ}$ (aq products) $-\Delta_{f} H^{\circ} \mathrm{Mg} \mathrm{SO}_{3} \cdot \mathrm{nH}_{2} \mathrm{O}(\mathrm{c})$. 
The tabulated $\Delta G^{\circ}(\mathrm{soln})$ for $\mathrm{MgSO}_{3} \cdot 6 \mathrm{H}_{2} \mathrm{O}=5.036 \mathrm{kcal} / \mathrm{mol}$ is obtained from the solubility, 0.062 moles $\mathrm{MgSO}_{3} / \mathrm{kg} \mathrm{H}_{2} \mathrm{O}$ given by [65SEI/LIN] and an estimated $\gamma_{ \pm}($at $I=0.25)=0.23$ obtained from the Davies equation (see $[68 \mathrm{HAM}])$. The calculated $\Delta \mathrm{S}^{\circ}(\mathrm{soln})$ results in $\mathrm{S}^{\circ} \mathrm{MgSO}_{3} \cdot 6 \mathrm{H}_{2} \mathrm{O}(\mathrm{c})=77 \mathrm{cal} /(\mathrm{mol} \cdot \mathrm{K})$, a reasonable value. $\mathrm{S}^{\circ} \mathrm{MgSO}_{3} \cdot 3 \mathrm{H}_{2} \mathrm{O}(\mathrm{c})$ and $\mathrm{S}^{\circ} \mathrm{MgSO}_{3}(\mathrm{c})$ are estimated and are used to obtain the other tabulated $\Delta G^{\circ}$ 's for the solution processes.

The Solution Process for $\mathrm{MgSO}_{4} \cdot \mathrm{nH}_{2} 2$ (c) (n=7,6,4,2,1,0) (reactions 77,79-85)

The $\Delta H^{\circ}$ (soln) of $\mathrm{MgSO}_{4}(\mathrm{c})$ in $\mathrm{H}_{2} \mathrm{O}$ is obtained from the $\Delta \mathrm{H}$ (soln) measurements of [83TH0], [83BER/IL0], and [85PIC] and the tabulated $\phi_{L}$ values for $\mathrm{MgSO}_{4}\left(\mathrm{nH}_{2} \mathrm{O}\right)$ at the appropriate concentrations.

The measurements of $[25 \mathrm{MAR}],[59 \mathrm{DEW} / \mathrm{RIC}]$, and [55KNO/STA] on the decomposition of $\mathrm{MgSO}_{4}(\mathrm{c})$ to $\mathrm{MgO}(\mathrm{c})$ and $\mathrm{SO}_{2}(\mathrm{~g})$ or $\mathrm{SO}_{3}(\mathrm{~g})$ lead to independent values for $\Delta_{f} H^{\circ} \mathrm{MgSO}_{4}(c)$ and $\Delta H^{\circ}$ (soln) which are in poor agreement.

The determination of $\Delta \mathrm{H}^{\circ}$ ( $(\mathrm{soln}) \mathrm{MgSO}_{4} \cdot \mathrm{H}_{2} \mathrm{O}(\mathrm{c})$ is based on the measurements of [56JAM/FRO], [83THO], and[85PIC]. The $\Delta H^{\circ}$ (soin) for $\mathrm{MgSO}_{4} \cdot \mathrm{H}_{2} \mathrm{O}$ (amorph) was also determined by [56JAM/FRO]. The $\Delta H^{\circ}$ (soln) of $\mathrm{MgSO}_{4} \cdot 2 \mathrm{H}_{2} \mathrm{O}(\mathrm{c}), \mathrm{MgSO}_{4} \cdot 4 \mathrm{H}_{2} \mathrm{O}(\mathrm{c})$ and $\mathrm{MgSO}_{4} \cdot 6 \mathrm{H}_{2} \mathrm{O}(\mathrm{c})$ were obtained primarily from the measurements of [83THO]. The $\Delta \mathrm{H}(\mathrm{Soln})$ of $\mathrm{MgSO}_{4} \cdot 7 \mathrm{H}_{2} \mathrm{O}$ as a function of concentration was measured by [51KAG/MIS] and [30PER]. These measurements as well as those of [52KAP/SAM], [83THO], and [85PIC] determined the $\Delta \mathrm{H}^{\circ}($ soln $)$ $\mathrm{MgSO}_{4} \cdot 7 \mathrm{H}_{2} \mathrm{O}(\mathrm{c})$.

[65SEI/LIN] lists the saturated solution to be $3.167 \mathrm{~mol} / \mathrm{kg} \mathrm{H} \mathrm{H}_{2} \mathrm{O}$ for $\mathrm{MgSO}_{4}(\mathrm{aq})$ in equilibrium with $\mathrm{MgSO}_{4} \cdot 6 \mathrm{H}_{2} \mathrm{O}(\mathrm{c}$ ) (metastable at $298 \mathrm{~K}$ ) and $3.026 \mathrm{~mol} / \mathrm{kg} \mathrm{H}_{2} \mathrm{O}$ in equilibrium with $\mathrm{MgSO}_{4} \cdot 7 \mathrm{H}_{2} \mathrm{O}(\mathrm{c})$. The activity coefficient and osmotic coefficient obtained from [59ROB/STO] are 0.0517 and 0.974 respectively at $3.167 \mathrm{~mol} / \mathrm{kg} \mathrm{H}_{2} \mathrm{O}$, and 0.0496 and 0.930 respectively at $3.026 \mathrm{~mol} / \mathrm{kg} \mathrm{H}_{2} \mathrm{O}$ so that $\Delta \mathrm{G}^{\circ}$ (soln) for the hexahydrate and heptahydrate are 2.539 and $2.735 \mathrm{kcal} / \mathrm{mol}$ respectively. 
The $\Delta G^{\circ}\left(\right.$ soln) and the $\Delta H^{\circ}($ soln) for the heptahydrate results in a calculated $\mathrm{S}^{\circ} \mathrm{MgSO}_{4} \cdot 7 \mathrm{H}_{2} \mathrm{O}(\mathrm{c})=88.5 \mathrm{cal} /(\mathrm{mol} \cdot \mathrm{K})$. In addition [40BEL] and [64KOH/ZAS] measured the $\mathrm{H}_{2} \mathrm{O}(\mathrm{g})$ decomposition pressure of the heptahydrate to the hexahydrate, resulting in values of 89.0 and $87.4 \mathrm{cal} /(\mathrm{mol} \cdot \mathrm{K})$, respectively. The $\triangle H$ obtained by [4OBEL] and [64KOH/ZAS] for this decomposition are consistent with the $\Delta H\left(S_{01 n}\right)$ measurements. The [64KOH/ZAS] measurements on the hexahydrate/tetrahydrate decomposition are reasonably consistent with the solution measurements. For the lower hydrate decomposition their measurements are not.

[64NEW] measured the $\triangle \mathrm{H}(\mathrm{soln})$ of $\mathrm{MgSO}_{4} \cdot 7 \mathrm{H}_{2} \mathrm{O}(\mathrm{c})$ and $3 \mathrm{Mg}(\mathrm{OH})_{2} \cdot \mathrm{MgSO}_{4} \cdot 8 \mathrm{H}_{2} \mathrm{O}(\mathrm{c})$ in $\mathrm{HCl}(\mathrm{aq})$. Combining these measurements with those of [38TAY/WEL] on the $\Delta \mathrm{H}(\mathrm{SO} / \mathrm{n})$ of $\mathrm{Mg}(\mathrm{OH})_{2}(\mathrm{c})$ in $\mathrm{HCl}(\mathrm{aq})$ results in $\Delta H^{\circ}=+2.1 \mathrm{kcal}$ for $3 \mathrm{Mg}(\mathrm{OH})_{2} \cdot \mathrm{MgSO}_{4} \cdot 8 \mathrm{H}_{2} \mathrm{O}(\mathrm{c}) \rightarrow \mathrm{MgSO}_{4} \cdot \mathrm{H}_{2} \mathrm{O}(\mathrm{c})+3 \mathrm{Mg}(\mathrm{OH})_{2}(\mathrm{c})$ $+\mathrm{H}_{2} \mathrm{O}(1)$.

The Dissociation Process for $\mathrm{MgSO}_{4}(20)$ (reaction 78)

The tabulated values are obtained from [58NAI/NAN]. 0ther values are listed by [64SIL/MAR].

The Solution Process for $\mathrm{MgCO}_{3} \cdot \mathrm{nH}_{2} \mathrm{O}$ (c) ( $\left.\mathrm{n}=5,3,0\right)$ (reactions 87-89)

[65LAN] reviewed the data on the stability relationships in the $\mathrm{MgO}-\mathrm{CO}_{2}-\mathrm{H}_{2} \mathrm{O}$ system. His interpretation and calculations were accepted for the $\Delta G^{\circ}\left(\right.$ soln) processes for $\mathrm{MgCO}_{3} \cdot \mathrm{H}_{2} \mathrm{O}(\mathrm{c})$ and $\mathrm{MgCO}_{3} \cdot 3 \mathrm{H}_{2} \mathrm{O}(\mathrm{c})$, and for the $\Delta G^{\circ}$ for decomposition of $\mathrm{MgCO}_{3} \cdot 3 \mathrm{H}_{2} \mathrm{O}(\mathrm{c})$. The value obtained from these and accepted for the $\Delta G^{\circ}(\mathrm{soln}) \mathrm{MgCO}_{3}(\mathrm{c})=7.0 \mathrm{kcal} / \mathrm{mol}$ is not in agreement with the $\log \mathrm{K}_{\mathrm{S}}=-7.46$ obtained by [62HAL]. The $\Delta \mathrm{H}^{\circ}(\mathrm{soln}) \mathrm{MgCO}_{3}(\mathrm{c})$ from the accepted $\Delta G^{\circ}(\mathrm{soln})$ and $\mathrm{S}^{\circ} \mathrm{MgCO}_{3}(\mathrm{c})$ is $=-11.50 \mathrm{kcal} / \mathrm{mol}$ which is supported 
by the measurements of [54KAP/STA] on the $\triangle \mathrm{H}$ (soln) measurements of $\mathrm{MgCO}_{3}(\mathrm{c})$ and $\mathrm{MgO}(\mathrm{c})$ which result in $\Delta H=23.5 \mathrm{kcal} / \mathrm{mol}$ for the decomposition of $\mathrm{MgCO}_{3}(\mathrm{c})$. The accepted $\Delta \mathrm{H}$ for this decomposition is $24.0 \mathrm{kcal} / \mathrm{mol}$ in agreement with [41ROT], but in sharp contrast with the measurements of [13MAR/SIM], [62ELL/MOR], and $[58 \mathrm{FOL} / \mathrm{KLI}]$, which indicate a $\Delta \mathrm{H} 2$ to $4 \mathrm{kcal} / \mathrm{mol}$ greater than the accepted. This system needs reinvestigation.

The Dissociation Process for $\mathrm{Mg}\left(\mathrm{HCO}_{3}\right)^{+}$(ao) (reaction 91)

[63HOS] reported the $\mathrm{pK}=0.95$ for the dissociation. 


\section{V-3. Processes Involving $\mathrm{Ca}$}

\section{The Formation Properties of $\mathrm{CaO}(\mathrm{c})$}

The $\Delta_{f} H^{\circ}$ is obtained from the combustion measurements of [56HUB/HOL].

The Relationship Between $\mathrm{CaO}(\mathrm{c})$ and $\mathrm{Ca}(\mathrm{OH})_{2}(\mathrm{c})$ (reaction 92)

This relationship is obtained from the measurements of [38TAY/WEL], [37WEL/TAY], and [35SCH/PRA] on $\triangle H^{\prime}$ 's of solution of $\mathrm{CaO}(\mathrm{c})$ and $\mathrm{Ca}(\mathrm{OH})_{2}(\mathrm{c})$ in $\mathrm{HCl}$ solutions. The measurements are in good agreement.

Solution Process for $\mathrm{Ca}(\mathrm{OH})_{2}(\mathrm{c})$ (reaction 93)

[65HOP/WUL] measured (and corrected for $\mathrm{CaOH}^{+}$(ao) formed) $\Delta \mathrm{H}(\mathrm{soln}$ ) $\mathrm{Ca}(\mathrm{OH})_{2}$. Their corrected value is $4.36 \mathrm{kcal} / \mathrm{mol}$. They also corrected the solubility $=.0203 \mathrm{~mol} / \mathrm{kg} \mathrm{H}_{2} \mathrm{O}$ [56BAT/BOW] for the presence of $\mathrm{CaOH}^{+}(\mathrm{ao})$, obtaining $\mathrm{m} \mathrm{Ca}^{+2}(\mathrm{aq})=0.0145, \mathrm{~m} \mathrm{OH}^{-}(\mathrm{aq})=0.0345, \mathrm{\gamma} \mathrm{Ca}^{+2}(\mathrm{aq})=0.507$ and $\gamma \mathrm{OH}^{-}(\mathrm{aq})=0.838$ (obtained from the extended Debye-Huckel relation), resulting in $\Delta G^{\circ}(\operatorname{soln})=7.11 \mathrm{kcal} / \mathrm{mol}$ or $\log \mathrm{K}_{S}^{\circ}=-5.21$. The tabulated values differ slightly from these because of the influence of other measurements on related compounds.

The Dissociation of $\mathrm{CaOH}^{+}(\mathrm{aO})$ (reaction 94)

The dissociation constant was determined by [59BAT/BOW], [54GIM/MON], [53BEL/GEO], and [5]DAV/HOY]. The values range from $K=0.07$ to 0.04 . $K=0.05$ was selected. [54GIM/MON] and [53BEL/GEO] also obtained $\Delta H^{\circ}=-1.25$ and $-1.19 \mathrm{kcal} / \mathrm{mol}$ respectively for this. [65HOP/WUL] obtained $\Delta \mathrm{H}^{\circ}=$ $-2.0 \mathrm{kcal} / \mathrm{nnol}$ from a least squares analysis of measurements on $\mathrm{Ca}\left(\mathrm{OH}_{2}(\mathrm{c})\right.$. Solution Process for $\mathrm{CaSO}_{3} \cdot 5 \mathrm{H}_{2}$ O(c) (reaction 96)

[23BIC] measured the solubility and reported $\Delta G^{\circ}($ soln $)=9.74 \mathrm{kcal} / \mathrm{mol}$ or $K_{S}^{\circ}=7.25 \times 10^{-8}$ assuming the dihydrate was the equilibrium solid phase. 
This value is assigned to the hemihydrate equilibrium phase. [70RAD] reports $\mathrm{K}_{\mathrm{S}}^{\circ}$ to be $8.4 \times 10^{-8}$ in fair agreement, with the solid phase the hemihydrate. The entropy of the hydrate is estimated as $29.0 \mathrm{cal} /(\mathrm{mol} \cdot \mathrm{K})$, so that with $\Delta S^{\circ} \simeq-41.4 \mathrm{cal} /(\mathrm{mol} \cdot \mathrm{K})$ and $\Delta G^{\circ}(\mathrm{soln}) \simeq 9.70 \mathrm{kcal} / \mathrm{mol}, \Delta H^{\circ}($ soln $)=$ $-2.3 \mathrm{kcal} / \mathrm{mol}$. This is a correction to TN270-6 [81NBS].

The Solution Process for $\mathrm{CaSO}_{4} \cdot \mathrm{nH}_{2} \mathrm{O}(\mathrm{c})$ ( $n=2,0.5$ and 0 ) (reactions 97-102) [46WAL], [25LAN/DUR], and [30LAN/MON] measured the $\triangle H$ (soln) of the dihydrate. They are in good agreement. The tabulated $\Delta G^{\circ}$ (soln) is based on $\mathrm{K}_{\mathrm{S}}{ }^{\circ}=4.22 \times 10^{-5}$ from [64SIL/MAR]. More recent evidence, [69GAR/GLU], [76LIL/BRI], and [75YOK/YAM] indicates that a better $\Delta G^{\circ}$ (soln) corrected for ion pairing, is $6.25 \mathrm{kcal} / \mathrm{mol}$ based on a solubility $=0.01518 \mathrm{~mol} / \mathrm{kg} \mathrm{H} \mathrm{H}_{2}$, $\gamma_{ \pm}=0.338$ and $a_{W}=0.9995$. This would result in $\Delta S^{\circ}(\mathrm{soln})=-21.9 \mathrm{cal} /(\mathrm{mol} \cdot \mathrm{K})$. [41 KEL/SOU] reviewed the relationships between the various hydrates. These were accepted. More recent measurements by [64MCA], on the $\triangle H$ 's of solution of the various hydrates, are in agreement with [41 KEL/SOU]. A reanalysis of the $\Delta G^{\circ}$ 's of solution will be made.

The Solution Process for $\mathrm{CaCO}_{3}$ (c, calcite) and (c, aragonite) (reactions 103, 104)

The decomposition pressure measurements of [36SAN], [56HIL/WIN], [36SOU/ROY], [33MAT/BIT], [60JAN/LOR], and [62BAK] on calcite, and the difference in the $\triangle \mathrm{H}^{\prime} \mathrm{s}$ of solution of $\mathrm{CaO}(\mathrm{c})$ and $\mathrm{CaCO}_{3}(\mathrm{c})$ measured by [63MEA/RIC], [25BAC], [41ROT/BER], [37WEL/TAY], and [28ROT/CHA] result in $\Delta H^{\circ}=42.62 \mathrm{kcal} / \mathrm{mol}$ for the decomposition to $\mathrm{CaO}(\mathrm{c})$ and $\mathrm{CO}_{2}(\mathrm{~g})$. The $\mathrm{K}_{\mathrm{s}}{ }^{\circ}$ measurements of [60GAR/THO], [60BIA/GHI], [58TRO], [29FRE/JOH], and [68HAL/TAS], are in good agreement and lead to the tabulated $\Delta G^{\circ}($ soln $)=11.33 \mathrm{kcal} / \mathrm{mol}$. However, more recent determinations, such as [74JAC/LAN] indicate that $\log K=-8.42$ 
$\left(\Delta G^{\circ}(\operatorname{soln})=11.49 \mathrm{kcal} / \mathrm{mol}\right)$ if ion pairing is ignored and

$\log K=-8.47\left(\Delta G^{\circ}(\mathrm{soln})=11.55 \mathrm{kcal} / \mathrm{mol}\right)$ if $\mathrm{CaCO}_{3}(\mathrm{ao})$ and $\mathrm{CaHCO}_{3}^{+}$(ao) are assumed. The $\Delta H^{\circ}($ soln $)$ with the latter approach is $-2.6 \mathrm{kcal} / \mathrm{mol}$ with $\Delta C_{p}=-109 \pm 2 \mathrm{cal} /(\mathrm{mol} \cdot \mathrm{K})$ and $\Delta S^{\circ}=-47.4 \pm 1.0 \mathrm{cal} /(\mathrm{mol} \cdot \mathrm{K})$. The values for the aragonite process are obtained from the transition properties calcite $\rightarrow$ aragonite, given by [35KEL/AND], [53JAM], and [56MAC]. 


\section{$\mathrm{V}-4$. Processes involving $\mathrm{Na}$}

The Solution Process for $\mathrm{NaOH}(\mathrm{c}), \mathrm{NaOH} \cdot \mathrm{H}_{2} \mathrm{O}$ (c) and $\mathrm{NaOH} \cdot \mathrm{nH}_{2} \mathrm{O}(1)$ (reactions 106-112)

The $\Delta H^{\circ}$ 's for the solution processes are taken from the measurements of [62MUR/GIA] who also measured the low temperature $C_{p}{ }^{\prime} s$ (corrected by [69SIE/GIA]). [69SIE/GIA] also measured the solubility of $\mathrm{NaOH} \cdot \mathrm{H}_{2} \mathrm{O}(\mathrm{c})$ to be $27.312 \mathrm{~mol} / \mathrm{kg} \mathrm{H} \mathrm{H}_{2} 0$. From [72HAM/WU] $\gamma_{ \pm}=31.88$ and $\phi=2.715$ resulting in $\Delta G^{\circ}(\operatorname{soln})=-6.438 \mathrm{kcal} / \mathrm{mol}$. The vapor pressure measurements are in agreement. The solution processes for the liquid $\mathrm{NaOH}$ hydrates are taken from the measurements of $[69 S I E / G I A]$.

The Formation of $\mathrm{NaSO}_{4}^{-}(20)$ (reaction 115)

[75REA], [50JEN/MON], and [69IZA/EAT] measured the $K$. The values are in good agreement. [62AUS/NAI] reported the $\Delta H$ (corrected to $\mu=0$ ).

The Solution Process for $\mathrm{Na}_{2} \mathrm{SO}_{3}(\mathrm{c})$ and $\mathrm{Na}_{2} \underline{\mathrm{SO}}_{3} \cdot 7 \mathrm{H}_{2} \underline{\mathrm{O}(\mathrm{c})}$ (reactions 113,114 )

The solid phase in equilibrium with the saturated solution of $\mathrm{Na}_{2} \mathrm{SO}_{3}$ (solubility $=2.42 \mathrm{~mol} / \mathrm{kg} \mathrm{H} \mathrm{H}_{2}$ ) is $\mathrm{Na}_{2} \mathrm{SO}_{3} \cdot 7 \mathrm{H}_{2} \mathrm{O}(\mathrm{c}$ ). With $\mathrm{\gamma} \pm=0.190$ and $\phi=0.738, \Delta G^{\circ}($ soln $)=0.96 \mathrm{kcal} / \mathrm{mol}$. From the extrapolation of the data above $308 \mathrm{~K}$ the solubility for the anhydrous $\mathrm{Na}_{2} \mathrm{SO}_{3}(\mathrm{c})$ would be $2.32 \mathrm{~mol} / \mathrm{kg}$ $\mathrm{H}_{2} \mathrm{O}$ with $\gamma_{ \pm}=0.191$ and $\phi=0.73$ so that $\Delta G^{\circ}$ for this process is $0.62 \mathrm{kcal} / \mathrm{mol}$. This solubility is now believed to be incorrect. The $\Delta G^{\circ}(\operatorname{soln})$ would most likely be close to zero. A reexamination is underway. $\Delta H^{\circ}=-3.55 \mathrm{kcal} / \mathrm{mol}$ for solution of the anhydrous salt from the measurements of [84FOR] who also measured the $\Delta H$ for the solution of the heptahydrate $\left(\Delta \mathrm{H}^{\circ}=+11.00 \mathrm{kcal} / \mathrm{mol}\right)$. The vapor pressure measurements of [28TAR] and [28ARI] on the heptahydrate 1 ead to $\Delta G^{\circ}=15.87$ and $15.36 \mathrm{kcal}$, respectively for the dehydration. All these values plus the measured $\mathrm{S}^{\circ} \mathrm{Na}_{2} \mathrm{SO}_{3}$ (c) (see $[61 \mathrm{KEL} / \mathrm{KIN}]$ ) were used to obtain the selected tabulated values. 
The Solution Process for $\mathrm{Na}_{2} \mathrm{SO}_{4}(\mathrm{c})$ and $\mathrm{Na}_{2} \mathrm{SO}_{4} \cdot 1 \mathrm{H}_{2} \mathrm{O}(\mathrm{c})$ (reactions 116,117 )

The $\Delta G^{\circ}$ soln $=1.677 \mathrm{kcal} / \mathrm{mol}$ for the decahydrate is obtained from the solubility $=1.96 \mathrm{~mol} / \mathrm{kg} \mathrm{H} \mathrm{H}_{2}$ [49ROS], $\gamma_{ \pm}=0.156$ and $\phi=0.621$ [59ROB $\left./ \mathrm{STO}\right]$. The $\Delta H^{\circ}(\operatorname{soln})=18.84 \mathrm{kcal} / \mathrm{mol}$ is obtained from the measurements of [38PIT/COU].

For the anhydrous $\mathrm{Na}_{2} \mathrm{SO}_{4}(c, v$, orthorhombic) the measurements of [69GAR/JEK] lead to $\Delta H^{\circ}=-0.58 \mathrm{kcal} / \mathrm{mol} . \Delta G^{\circ}$ for the solution process $=+0.420 \mathrm{kcal} / \mathrm{mol}$ is obtained from $\mathrm{m}=3.63 \mathrm{~mol} / \mathrm{kg} \mathrm{H}_{2} \mathrm{O}$ [49ROS] and $\gamma_{ \pm}=0.137$ from $[59 R 0 B /$ STO $]$.

In addition to these measurements, the $\mathrm{H}_{2} \mathrm{O}$ vapor pressure measurements of [37HIG] and [29MAT/OGU] on the decahydrate result in $\Delta G^{\circ}=21.79 \mathrm{kcal} / \mathrm{mol}$ $\mathrm{Na}_{2} \mathrm{SO}_{4} \cdot 10 \mathrm{H}_{2} \mathrm{O}(\mathrm{c})$. The tabulated values result from this input data. The $\mathrm{S}^{\circ}$ $\mathrm{Na}_{2} \mathrm{SO}_{4} \cdot 10 \mathrm{H}_{2} \mathrm{O}(\mathrm{c})$ includes a $1.08 \mathrm{cal} /(\mathrm{mol} \cdot \mathrm{K})$ correction for a zero point entropy. 
VI Documentation and Discussion of the Aqueous Solution Property Values The arrangement is as follows:

VI-1. A general description of the methods and equations used to obtain $\gamma_{ \pm}$, the mean ionic activity coefficient, and $\phi$. the osmotic coefficient is described. Included are the concentration ranges for which the equations used for $\gamma_{ \pm}$and $\phi$ are valid.

VI-2. Some specific sources of information for $\phi_{L}$, the relative apparent molar enthalpy, is provided for $\mathrm{FeSO}_{4}, \mathrm{MgSO}_{4}, \mathrm{CaSO}_{4}, \mathrm{Na}_{2} \mathrm{SO}_{3}$, and $\mathrm{Na}_{2} \mathrm{SO}_{4}$, as well as comments on $\gamma_{ \pm}$at saturation for these substances. 


\section{VI-1. Activity and 0smotic Coefficients}

Activity and osmotic coefficients can be calculated using various semi-theoretical equations. There have been two major efforts towards the evaluation of activity and osmotic coefficients during the past 10 years. Pitzer and coworkers have formulated a set of equations of the virial coefficient type consistent with both the modern statistical mechanical description of electrolyte solutions and the basic principles of thermodynamics of solutions. The Electrolyte Data Center at NBS (see [78GOL/NUT]) has completed many detailed critical evaluations of electrolytes using an extensive data base of all available experimental data and has used extended forms of the Lietzke-Stoughton-Hamer-Wu equations [62LIE/STO,72HAM/WU], and more recently, extensions of the Pitzer equations [74PIT/MAY]. Both of these types of equations have been employed in the representation of activity data here.

For this report, the activity and osmotic coefficient of $2: 2$ charge type electrolytes $\left(\mathrm{MgSO}_{4}, \mathrm{CaSO}_{4}\right.$, and $\left.\mathrm{MnSO}_{4}\right)$ have been calculated from the Pitzer equations.

For the activity coefficient of these $2: 2$ salts, the equation is

$$
\ln \gamma=4 f^{\gamma}+m B_{M X}^{\gamma}+m^{2} C_{M X}^{\gamma}
$$

where

$$
f^{\gamma}=-A_{\phi}\left[I^{1 / 2} /\left(1+b I^{1 / 2}\right)+(2 / b) \ln \left(1+b I^{1 / 2}\right)\right]
$$

and

$$
\begin{aligned}
B_{M X}^{\gamma} & =2 \beta_{M X}^{(0)}+\left(2 \beta_{M X}^{(1)} / \alpha_{1}^{2} I\right)\left[1-\left(1+\alpha_{1} I^{1 / 2}-\frac{1}{2} \alpha_{1}^{2} I\right) e^{\left.-\alpha_{1} I^{1 / 2}\right]}\right. \\
& +\left(2 \beta_{M X}^{(2)} / \alpha_{2}^{2} I\right)\left[1-\left(1+\alpha_{2} I^{1 / 2}-\frac{1}{2} \alpha_{2}^{2} I\right) e^{\left.-\alpha_{2} I^{1 / 2}\right]}\right.
\end{aligned}
$$

and

$$
C_{M X}^{\gamma}=\frac{3}{2} C_{M X}^{\phi}
$$

The osmotic coefficient equation is

$$
\phi-1=4 f^{\phi}+m B_{M X}^{\phi}+m^{2} C_{M X}^{\phi}
$$


where

$$
f^{\phi}=-A_{\phi}\left[I^{1 / 2} /\left(1+b I^{1 / 2}\right)\right]
$$

and

$$
B_{M X}^{\phi}=\beta_{M X}^{(0)}+\beta_{M X}^{(1)} e^{-\alpha_{1} I^{1 / 2}}+\beta_{M X}^{(2)} e^{-\alpha_{2} I^{1 / 2}}
$$

Here, $I$ is the ionic strength, $1 / 2 \Sigma \mathrm{mz}_{i}{ }^{2}$ and $M X$ is the general salt formed from the $M$ ions. Definition of terms and a more detailed discussion of these equations are given in reference [74PIT/MAY]; parameters for these equations are given in the appropriate tables (section IV).

For the uni-univalent compounds, and the unsymmetrical charge-types, uni-bi and bi-univalent compounds, the activity and osmotic coefficients have been calculated from the Hamer-Wu, Lietzke-Stoughton equations used in previous evaluations [77STA/NUT].

The equation for the osmotic coefficient is:

$$
\begin{aligned}
\emptyset & =1+\left\{| z _ { + } z _ { - } | A _ { m } \left[-\left(1+B I^{1 / 2}\right)+21 n\left(1+B I^{1 / 2}\right)\right.\right. \\
& \left.+1 /\left(1+B I^{1 / 2}\right]+1 / 2 C m+2 / 3 D m^{2}+3 / 4 \mathrm{Em}^{3}+\ldots\right\}
\end{aligned}
$$

and the activity coefficient:

$$
\ln \gamma_{ \pm}=\frac{-\left|z_{+} z_{-}\right| A I^{1 / 2}}{1+B I^{1 / 2}}+C m+D m^{3 / 2}+E m^{2}+\ldots(V I-9)
$$

The parameters for these equations are tabulated in the appropriate tables in section IV. Activity coefficients for these charge-types may also be calculated from the Pitzer equations for the uni-univalent and uni-bi and bi-univalent salts. In these cases, the Pitzer equations are sometimes applicable to a more limited concentration range. Each table references the sources of data.

Caution should be exercised in the use of these equations because the equations are only valid over a specific concentration range. The valid ranges of concentrations are summarized in Table VI-l. 


\section{Table VI-1.}

Limitations on the Use of Activity and Osmotic Coefficient

Equations at $298 \mathrm{~K}$.

Compound

$\mathrm{H}_{2} \mathrm{SO}_{4}$

$\mathrm{MnSO}_{4}$

$\mathrm{MgSO}_{4}$

$\mathrm{CaSO}_{4}$

$\mathrm{Na}_{2} \mathrm{SO}_{3}$

$\mathrm{Na}_{2} \mathrm{SO}_{4}$

$\mathrm{KOH}$

$\mathrm{K}_{2} \mathrm{SO}_{4}$
Valid Concentration Range

$\mathrm{mol} / \mathrm{kg} \mathrm{H}_{2} \mathrm{O}$

$0.001-28.0$

$0.7-4.0$

$0.006-3.0$

$0.004-0.0011$

$0-2.0$

$0-4.5$

$0-20.0$

$0-0.7$ 
VI-2. The Relative Apparent Molar Enthalpy, $\phi_{L}$

All values for $\Delta_{f} H$ as a function of concentration are obtained from the tabulations in [81NBS]. $\phi_{L}$ is retrieved from the relationship:

$$
\phi_{L}=\Delta_{f} H\left(M X\left(n H_{2} O\right)\right)-\Delta_{f} H^{\circ}(M X(a))
$$

where $\Delta_{f} H^{\circ}$ is the enthalpy of formation in the standard state. See section I-2.4 for conventions used in defining $\Delta_{f} H^{\circ}$ for electrolytes.

Normally, for simple salts and acids, $\phi_{L}$ is the negative of the enthalpy of dilution to infinite dilution. However, for salts such as $\mathrm{NaHSO}_{4}$, having an ion that can itself ionize, $\phi_{L}$ differs from the enthalpy of dilution to infinite dilution by $\Delta H$ for the ionization of the acid anion.

Specific sources for $\phi_{L}$ for some systems follows.

$\mathrm{FeSO}_{4}$

The dilution values have been obtained from the various solution reactions involving $\mathrm{Fe}^{+2}$ aqueous solutions with $\mathrm{SO}_{4}{ }^{-2}$ aqueous solutions measured by [83THO] and [ 1873BER].

$\mathrm{MgSO}_{4}$

As indicated under the section for the solution process for $\mathrm{MgSO}_{4} \cdot \mathrm{nH}_{2} \mathrm{O}$ (c) [51KAG/MIS] and [3OPER] determined the $\Delta \mathrm{H}(\mathrm{soln})$ of $\mathrm{MgSO}_{4} \cdot 7 \mathrm{H}_{2} \mathrm{O}(\mathrm{c})$ as a function of concentration. These measurements were converted to $\Delta H^{\prime} s($ di $7 n$ ) and merged with the measured $\Delta H^{\prime} s$ (diln) of [28LAN], [31LAN/STR], [32PLA], [27LAN/MES], [27LAN/MES], and [83THO] to obtain a smooth curve for $\phi_{L^{\cdot}}$. The extrapolation to infinite dilution was made to follow as closely as possible the limiting Debye-Huckel slope. The $\Delta H$ measurements of [83THO] on the $\Delta H$ (mix) of aqueous solutions of $\mathrm{MgCl}_{2}$ or $\mathrm{Mg}\left(\mathrm{NO}_{3}\right)_{2}$ with coefficients have been calculated from [74PIT/MAY] and at $m=3.167 \mathrm{~mol} / \mathrm{kg} \mathrm{H}_{2} 0, \gamma_{ \pm}$is 0.0569 which differs slightly from the value cited in section $V$ from [81NBS], (0.0517). Similarly, the activity of water differs, 0.964 compared with 0.974 in section $V$ taken from TN 270-6 [81NBS]. These differences will be resolved. 
The enthalpies of dilution were obtained from the measurements of [30LAN/MON] and [31LAN/STR]. The activity coefficients have been calculated from [74PIT/MAY]. The activities at saturation, $0.01518 \mathrm{~mol} / \mathrm{kg} \mathrm{H}_{2} 0$, agree well (0.331) when compared to 0.338 cited in section $V-4$ from [81NBS].

$\mathrm{Na}_{2}{ }_{-3}$ and $\mathrm{Na}_{2} \mathrm{SO}_{4}$

The dilution heats for $\mathrm{Na}_{2} \mathrm{SO}_{3}$ are based on the neutralization measurements of [83THO] and [31RAM/HAN] with $\mathrm{NaOH}(\mathrm{aq})$.

The $\phi_{L}$ values for $\mathrm{Na}_{2} \mathrm{SO}_{4}$ are based on the measurements of [77SOL/VLA], [74THO/SMI], [4TWAL/ROB], and [58BRO/GIA].

The activities calculated from [81GOL] agree we 11 with those used in [81NBS] for saturated solutions of $\mathrm{Na}_{2} \mathrm{SO}_{3}$ and $\mathrm{Na}_{2} \mathrm{SO}_{4}$. 
(18--)

53FAU/SIL FAURE, P.-A.; SILBERMAN, J.T.; ANN. CHIM. PHYS.; [3], 37, 406 (1853)

63PAP

65K OP

$71 D I T$

71 MAR

73BER

75BER

TGMAR

TGMAR

7 SMAR

83EER/ILO

$83 \mathrm{THO}$

84 BER

$84 F O R$

B4FOR2

S5PIC

EEPIC

87EER

ETFRO

87HAR

ETP IC

$96 \mathrm{CHA}$

У9POL

(19--)

O1COH/VIS

OTRUM

$12 N O Y / B R A$

13MAR / SIH

$13 R O L / A C C$

14BIL

$1 \triangle F O R$

$14 F O R 2$

$14 F O R 3$

$18 \mathrm{SMI}$

ट3BIC

ZUCAR/JET

$24 F O E / B R O$

25BAC

2SBAC

2SLAN/DUR

25MAR
PAPE, C.; ANN. PHYS. (LEIPZIG); [2], 120, 337 (1863) KOPP, H.; PHILOS. TRANS. ROY. SOC. LONDON; 155, 71 (1865) DITTE, A.; C. R. ACAD. SCI.; 72, 762 (1871) MARIGNAC, J.-C.G.; BIBL. UNIU. ARCHIVES; 42, 209 (1871) BERTHELOT, M.; ANN. CHIM. PHYS.; [4], 30, 145 (1873) BERTHELOT, M.; ANN. CHIM. PHYS.; [5], 4, 160 (1875) MARIGNAC, C.; ANN. CHIM. PHYS.; [5], 8, 410 (1876) MARIGNAC, C.; ARCH. SCI. PHYS. NAT.; 【2], 55, 113 (1876) MARIGNAC, C.; ANN. CHIM. PHYS.; [5], 8, 410 (1878) EERTHELOT, M.; ILOSUAY, L.; ANN. CHIM. PHYS.; [5], 29, 295 (1883)

THOMSEN, J.; 'THERMOCHEMISCHE UNTERSUCHUNGEN' VOL. I- VOL. IV, J. BARTH VERLAG, LEIPZIG; (1882-1886) BERTHELOT, M.; ANN. CHIM. PHYS.; [6], 1, 73 (1884) DEFORCRAND, R.; ANN. CHIM. PHYS.; [6], 3, 242 (1884) DEFORCRAND, R.; COMPT. REND. ACAD. SCI.; 98, 738 (1884) PICKERING, S. U.; J. CHEM. SOC.; 47, 100 (1885) PICKERING, S.U.; J. CHEM. SOC.; 49, 260 (1886) BERTHELOT, M.; ANN. CHIM. PHYS.; [6], 11, 310 (1887) FROWEIN, P. C. F.; Z. PHYS. CHEM.; 1, 5 (1887) HARTOG, P.-J.; C. R. ACAD. SCI.; 104, 1763 (1887) PICKERING, S. U.; J. CHEM. SOC.; 51, 290 (1887) LECHATELIER, H.; C. R. ACAD. SCI.; 122, 80 (1896) PQLLOCK, J. H.; CHEM. NEWS; 79,56 (1899)

COUEN, E.; VISSER, A. W.; Z. PHYS. CHEM.; 36, 517 (1901) RUMELIN, G.; Z. PHYS. CHEM.; 58, 449 (1907)

NUYES, A. A.; BRANN, B. F.; J. AM. CHEM. SOC.; 34, 1016 $(1912)$

MARE, R.; SIMEK, A.; A. ANORG. ALLG. CHEM.; 82, 17 (1913) ROLLA, L.; ACCAME, L.; ATTI. ACCAD. NAZL. LINCEI. REND., CL. SCI. FIS. MAT. NAT.; [5], 22, 109 (1913)

EILTZ, W.; Z. ANORG. ALLG. CHEM.; 89, 141 (1914)

DEFORCRAND, R.; C. R. ACAD. SCI.; 158, 20 (1914)

DEF ORCRAND, R.; C.R. ACAD. SCI.; 158, 843 (1914)

DEFURCRAND, R.; C. R. ACAD. SCI.; 158, 991 (1914)

SMITH, H. J.; J. AM. CHEM. SOC.; 40, 879 (1918)

BICHOWSKY, R.; J. AM. CHEM. SOC.; 45, 2225 (1923)

CARPENTER, C. D.; JETTE, E. R.; J. AM. CHEM. SOC.; 45, 578 (1923)

FOERSTER, F.; BROSCHE, A.; NORBERG-SCHULZ, C.; Z. PHYS.

CHEM.; $110,435(1924)$

BACKSTROM, H. L. J.; J. AM. CHEM. SOC.; 47, 2432 (1925)

BACKSTROM, H. L. J.; J. AM. CHEM. SOC.; 47, 2443 (1925)

LANGE, E.; DURR, F.; Z. PHYS. CHEM.; 118, 129 (1925)

MARCHAL, G.; J. CHEM. PHYS.; 22, 493 (1925) 
26STI/FEL STILES, A. G.; FELSING, W. A.; J. AM. CHEM. SOC.; 48,1543 (1926)

27FRA/HEI FRANCK, H.; HEIMANN, H.; Z. ELEKTROCHEM.; 33, 469 119271 27LAN/MES LANGE, E.; MESSNER, G.; Z. ELEKTROCHEM.; 33, 431 (1927) 27LAN/MES2 LANGE, E.; MESSNER, G.; NATURWISSENSCHAFTEN; 15,52111721, 2\&LAN LANGE, E.; FORTSLHR. CHEM., PHYSIK U. PHYSIK. CHEM.; NO.6, 19,1 (1928)

28 AR I

28LOR/WOO

ARII, K.; BULL INST. PHYS. CHEM RESEARCH; 1,891 (1928) LORENZ, R.; WOOLCOCK, J.; Z. ANORG. ALLG. CHEM.; 176, 289 (1928)

28ROT/CHA ROTH, W. A.; CHALL, P.; Z. ELEKTROCHEM.; 34, 185 (1928; 2QTAR

29FRE/JOH TARASENKOU, 407 (1928) 29GIB/DRI GIBSON, G. C.; DRISCOLL, J.; JONES, W. J.; J. CHEM. SOC.; FREAR, G.L.; JOHNSTON, J.; J. AM. CHEM. SOC.; 51, 2082

29MAT/OGU MATSUI, M.; QQURI, S.; KAMBARA, S.; KATO, K.; KOGYO KAGAKU ZASSHI; 32,1728 (1929)

29POP/KUN POPOFF, S.; KUNZ, A. H.; J. AM. CHEM. SOC.; 51, 382 (1929) 29ROT

29ROT/MUL ROTH, W. A.; ARCH. EISSENHUTTENW.; 3, 339 11929;

29TRA/PAK TRAUTZ, M.; PAKSCHWER, 5.; J.PRAKT. CHEM.; [2], 122, 147 (1929)

3OLAN/MON

3OPER

LANGE, E.; MONHEIM, J.;

Z. PHYS. CHEM.;

A150, $349(1930)$

PERREU, J.; C. R. ACAD. SCI.; 190, 429 (1930)

31LAN/STR

LANGE, E.; STREEK, H.

Z. PHYS. CHEM. (LEIPZIG); A157, 1 (1931)

$31 M O R / M A A$ MORGAN, 0. M.; MAASS, 0.; CAN. J.RES.; 5, 162 (1931)

$31 P O P / F L E$

31 RAM/HAN FOPOFF, S.; FLEHARTY, U. B.; HANSON, E. L.; J. AM. CHEM. SOC.; $53,1643(1931)$

RAMSTETTER, H.; HANTKE, G.; Z. PHYS. CHEM. BODENSTEIN FESTBAND; $662(1931)$

32BRI/ROB BRITTON, H. T. S.; ROBINSON, R. A.; TRANS. FARDAY SOC.; 28,531 (1932)

32FLA

3ZRAN/FRA PLAKE, E.; Z. PHYS. CHEM. (LEIPZIG); A162, 257 (1932)

¿ROT/ZEU

32ZAW

RANDALL, M.; FRANDSEN, M.; J. AM. CHEM. SOC; $54,47(1932)$ ROTH, W. A.; ZEUMER, H.; Z. ELEKTROCHEM.; 38, 164 (1932) ZAWADZKI, J.; Z. ANORG. U. ALLGEM. CHEM.; 205, 180 (1932) 3ZLAN/MON LANGE, E.; MONHE

33LAT/HIC LATIMER, W. M.; HICKS, J.F. G., JR.; SCHULTZ, F.; J. CHEM. PHYS.; $1,620(1933)$

3.3MAT/BIT MATSUI, M.; BITO, K.; MURAYANA, S.; KADONO, M.; J. SOC.

34 AND

$\begin{array}{ll}34 A N D & \text { ANDERSON, C. T.; J. AM. CHEM. SOC.; 56, 849 (1934) } \\ 34 \text { BRA/HER BRAY, W. C.; HERSHEY, A. U.; J. AM. CHEM. SOC.; 56, } 1889\end{array}$ CHEM. IND. JFN.; 36, 155 (1933)

ANDERSON, C. T.; J. AM. CHEM. SOC.; 56, 340 (1934)

$34 \mathrm{JOH}$ LLEP JOHNSTONE, H.F.; LEPPLA, P.W.; J. AM. CHEM. SOC.; 63, $808(1941)$ 
$34 P E R$

35DRU

35GRO/SCH

35GUR/GIS

35KAM

35KEL/AND

$35 P E R$

$35 S C H / P R A$

35SCH/SWE

3 $6 H E R / B R A$

3 GFER

36ROE/JON

3GSAN

36SOU/ROY

$370^{\circ} \mathrm{A} / T O L$

$376 I A / A R E$

$37 H I G$

37KEL

3 TNEU/HEI

37SCH/SHE

37 WEL/TAY

38NEW/WEL

3BPIT/COU

उBTAY/WEL

3BZDA/SUS

$39 P E R$

39UOL

$40 B E L$

$40 U L I / S I E$

$\triangle 1 \mathrm{KAP}$

$41 \mathrm{KEL} / S O U$

41ROT

A IROT/EER

41TAR/GAR

41 WAL/ROB

$42 M 00 / K E L$

PERREU, J.; C. R. ACAD. SCI.; 198, 1410 (1934)

DRUCKER, C.; ARK. KEM. MINERAL. GEOL.; 11A, 18 (1935)

VONGRUNOW, H.; SCHWEITE, H. E.; ZEMENT.; 24,271 (1935)

GURD, G. W.; GISHLER, P. E.; MAASS, Q.; CAN. J. RES.;

13,209 (1935)

KAMIIKE, 0.; BULL. INST. PHYS. CHEM. RES. (TOKYO); 14, 163 (1935)

KELLEY, K. K.; ANDERSON, C. T.; U.S. BUR. MINES.; BULL. 384, (1935)

PERREU, J.; C. R. ACAD. SCI.; 200, 237 (1937)

SCHWIETE, H. E.; PRANSCHKE, A.; ZEMENT; 24, 593 (1935)

SCHUMB, W. C.; SWEETSER, S. B.; J. AM. CHEM. SOC.; 57, 871 (1935)

HERSHEY, A. U.; BRAY, W. C.; J. AM. CHEM. SOC.; 58, 1760 (1936)

PERREU, J.; THESIS; (1936)

ROBINSON, R. A.; JONES, R. S.; J. AM. CHEM. SOC.; 58, 959 (1936)

SANO, K.; J. CHEM. SOC. JPN.; 57, 1025 (1936)

SOUTHARD, J. C.; ROYSTER, P. A.; J. PHYS. CHEM.; 40,435

(1936)

D'ANNS, J.; TOLLERT, H.; Z. ELEKTROCHEM.; 43, 81 (1937)

GIAUQUE, W.F.; ARCHIBALD, R.C.; J. AM. CHEM. SOC.; 59, 561 (1937)

HIGUCHI, T.; J. CHEM. SOC. JAPAN; 58, 193 (1937)

KELLEY, K. K; U.S. BUR. MINES; BULL. $406,154 \mathrm{PP}$

(1937)

NEUMANN, B.; HEINTKE, G.; Z. ELEKTROCHEM.; 43, 246 (1937)

SCHUMB, W. C.; SHERRILL, M. S.; SWEETSER, S.; J. AM. CHEM. SOC.; 59, 2360 (1937)

WELLS, L. S.; TAYLOR, K.; J.RES. NATL. BUR. STAND.; 19, 215 (1937)

NEWMAN, E. S.; WELLS, L. 5.; J. RES. NATL. BUR. STAND.;

$20,825(1938)$

PITZER, K. S.; COULTER, L. V.; J. AM. CHEM. SOC.; 60, 1310 (1938)

TAYLOR, K.; WELLS, L. S.; J. RES. NATL. BUR. STAND.; 21, 133 (1938)

ZDANUUSKII, A. B.; SUSLINA, K. D.; Z. FIZ. KHIM.; 12, 109 (1938)

PERREU, J.; C. R. ACAD. SCI.; 209, 311 (1939)

VOLZHENSKII, A. U.; ZH. PRIKL. KHIM.; 12, 360 (1939)

BELL, J.; J. CHEM. SOC.; (1940), 72

ULICH, H.; SIEMUNSEN, H.; ARCH. EISENHUTTEN.; 14, $27(1940)$

KAPUSTINSKII, A. F.; ACTA PHYSICOCHIM. URSS; 14, 503 (1941)

KELLEY, K. K.; SOUTHARD, J. C.; ANDERSON, C. T.; U.S. BUR.

MINES; EULL. 625, (1941)

ROTH, W. A.; J. PRAKT. CHEM.; [2], 158, 117 (1941)

ROTH, W. A.; BERENDT, H.; WIRTHS, G.; Z. ELEKTROCHEM.;

$47,185(1941)$

TARTAR, H. V.; GARRETSUN, H. H.; J. AM. CHEM. SOC.; 63, 808 (1941)

WALLACE, W.E.; ROEINSON, A.L.; J. AM. CHEM. SOC.; 63, 958 (1941)

MOORE, G. E.; KELLEY, K. K.; J. AM. CHEM. SOC.; 64, 2949 (1942) 
$42 S O U / S H O$

43SHO/HUF

4 4KEL/MOO

45VOS/YAN

$46 F O N$

46WAL

ATEVA

4ETOR/SAH

48WAL

49EVA/BAX

$49 G I A$

$49 R \cup B / S T O$

49R0S

SOJEN/MON

SUPER

$5 O P L U$

$51 \mathrm{CON} / \mathrm{MCV}$

51DAU/HOY

51 DUN/JAM

S1HOL/HUE

51KAG/MIS

$51 \mathrm{KOB}$

$515 M I$

5ZEAI/PAT

$52 I S I / K I G$

52KAF/SAM

53BEL / GEO

53BOC/HER

53 JAM

$53 \mathrm{JON} / \mathrm{MON}$

$53 L E U / K O L$

53MAG/HUI

5.3PAT/THO

54 GIM/MON

S $4 K A F / S T A$
SUUTHARD, J. C.; 64,1770 (1942)

SHOMATE, C.H.; 1626 (1943)

KELLEY, K. K. (1944)

VOSKRESENSKAYA, N. K.; YANKOUSKAYA, G. N.; IZV. AKAD. NAUK. SSSR, OTD. KHIM. NAUK.; 1, 3 (1945)

FONTATA, B. J.; U.S. AEC RPT.; MDDC-542, (1946)

WALLACE, W. E.; J. PHYS. CHEM.; 50, 152 (1946)

EVANS, M. W.; U.S. AEC RPT.; MDDC-1206, (1947)

TURGESUN, D.R.; SAHAMA, TH. G.; J. AM. CHEM. SOC; 70,2156 (1948)

WALKLEY, A.; J. ELECTROCHEM. SOC.; 93, 316 (1948)

EUANS, M. G.; BAXENDALE, J. H.; URI, N.; TRANS. FARADAY SUC.; 45,236 (1949)

GIAUQUE, W. F.; J. AM. CHEM. SOC.; 71, 3192 (1949)

ROBINSON, R. A.; STOKES, R. H.; TRANS. FARADAY. SOC.;

45,612 (1949)

ROSENEERG, T.; ACTA. CHEM. SCAND.; 3, $50(1949)$

JENKINS, I. L.; MONK, C. B.; J. AM. CHEM. SOC.; 72, 2695 (1950)

PERRY'S CHEM. ENG. HANDBOOK, THIRD ED.; MCGRAW HILL, N.Y.; 676 (1950)

PLUMMER, A. W.; CHEM. ENG. PROG.; 46, 369 (1950)

CONNICK, R. E.; MCUEY, W. H.; J. AM. CHEM. SOC.; 73, 1798

(1951)

DAUIES, C. W.; HOYLE, B. E.; J. CHEM. SOC.; (1951), 233 DUNSMURE, H. S.; JAMES, J. C.; J. CHEM. SOC.; (1951), 2925 HOLLEY, C. E.; HUBER, E. J.; J.AM. CHEM. SOC.; 73, 5577 (1951)

KAGANOUICH, YU. YA.; MISCHENKO, K. P.; Z. OBSCHEI. KHIM.; 21,28 (1951)

KUBAYASHI, K.; SCI. RPTS. TOHOKU IMF. UNIV.; [1], 35, 111 (1951)

SMITH, G. F.; ANAL. CHEM.; 23, 925 (1951)

EAILEY, F. E., JR.; PATTERSON, A., JR.; J. AM. CHEM. SOC.; 74,4426 (1952)

ISIHARA, T.; KIGOSHI, A.; BULL. RES. INST. MIN. DRESS. METALL. (SENDAI); 8, 83 (1952)

KAFUSTINSKII, A. F.; SAMUILOU, 0. YA.; ZH. FIZ. KHIM.; 26, 918 (1952)

BELL, R. P.; GEERGE, J. B.; TRANS. FARADAY. SOC.; 49, 619 (1953)

BOCK, R.; HERRMANN, M.; Z. ANURG. ALLG. CHEM.; 273, 1 (1953) JAMESON, J. L.; J. CHEM. PHYS.; 21, 1385 (1953)

JUNES, W. H.; MONK, C. B.; TRANS. FARADAY. SOC.; 49, 619 (1953)

LEUSSING, D.L.; KULTHUFF, I.M.; J.AM. CHEM. SOC.; 75, 2476 (1953)

MAGNUSSON, L. B.; HUIZENGA, J.R.; J. AM. CHEM. SOC.; 75, 2242 (1953)

PATRICK, W. A.; THOMPSON, W. E.; J. AM. CHEM. SOC.; 75, 1184 (1953)

GIMBLETT, F.R.; MONK, C. M.; TRANS. FARADAY SOC.; 49, 619 (1954)

KAFUSTINSKII, A. F.; STAKHANOUA, M. S.; IZV. AKAD. NAUK. SSSR, OTD. KHIM. NAUK.; 587 (1954) 
S5KNU/STA

$55 \mathrm{SCH} / W E I$

56BAT/BOW

56GAY/WOO

$56 \mathrm{HIL} / W I N$

56 HUB/HOL

$56 \mathrm{JAM} / F R O$

$56 M A C$

$56 T S C / P I E$

ᄃ.7BER/UIN

$57 B U N$. NYE

57CLA

S7CUT/BER

57FRO/BRE

57GOL/GRA

58BRO/GIA

$58 \mathrm{FOL} / \mathrm{KLI}$

58KAP/SHI

5\&KOR/VER

SBLAP

5. 8 MAR

SBNAI/NAN

SBSTR/PEE

SBTRO

S9BAT / BOW

59BUR: / ABB

S9DEW/RIC

SFFUR/REI

59KUE/COU

57MAT

S9MAU

59NAI/NAN

59RUB/STO

6OBEW

GOBIA/CHI

KNOPF, H. J. 265 (1955)

SCHEDLING, J.A.; WEIN, J.; OSTER. AKAD. WISS. MATH.NATURALWISSEN KL. SITZBER. ABT.; IIA, 164, 175 (1955)

BATES, R. G.; BOWER, U.E.; SMITH, E.R.; J. RES. NBS; 56, 305 (1956)

GAYER, K. H.; WONTNER, L.; J. PHYS. CHEM.; 60, 1569 (1956) HILL, K. J.; WINTER, E. R. S.; J. PHYS. CHEM.; 60, 1361 (1956)

HUBER, E. J.; HOLLEY, C.E.; J. PHYS. CHEM.; 60, 498 (1956) JAMIESON, J.W. S.; FROST, G. B.; CAN. J. CHEM.; 34,583 (1956)

MACDONALD, G. J.F.; AM. MINERALOG.; 41, 744 (1956)

TSCHAPPAT, C.; PEICE, R.; HELU. CHIM. ACTA.; 39, 1427 (1956) BERGSTEIN, A.; UINTERA, J.; COLL. CZECH. CHEM. COMMUNS.; 22, 884 (1957)

BUNCE, J. L.; NYE, J. D.; E.K. AEA AERE COMPT. REND.; 2410 , 4PF (1957)

CLARK, 5. P., JR.; AM. MINERALOG.; 42, 564 (1957)

EUTA, F.; BERANEK, E.; PISECKY, J.; CHEM. LISTY; 51, 1614 (1957)

FROST, G.B.; BREEK, W.G.; CLAYTON, R.N.; REDDOCH, Q.H.; MILLER, G.G. CAN. J. CHEM.; 35, 1446 (1957)

GOLDSMITH, J. L.; GRAF, D. L.; GEOCHIM. COSMOCHIM. ACTA.; 11,312 (1957)

BRÓdALE, G.; GIAUQUE, W. F.; J. AM. CHEM. SOC.; 80, 2042 (1958)

FOLDUARI, M.; KLIBURSZKY, B.; ACTA. GEOL. ACAD. SCI. HUNG.;

5,187 (1958)

KAPUSTINSKII, A. F.; SHIDLOUSKII, A. A.; SHIDLOUSKAYA, YU. S.; IZU. AKAD. NAUK. SSSR, OTD. KHIM. NAUK.; 385 (1958)

KOR, S. K.; VERMA, G. S.; J. CHEM. PHYS.; 29, 11 (1958) LAPTEVA, 0. N.; ZHUR. PRIKLAD. KHIM.; 31, 1210 (1958) MARCUS, Y.; J. PHYS. CHEM.; 62, 1314 (1958)

NAIR, U. S. K.; NANCOLLAS, G. H.; J. CHEM. SOC.; (1958), 3706

STROMATT, R. W.; PEEKEMA, R. M.; SCOTT, F. A.; U.S. AEC RFT.; HW-58212; (1958)

TRUILIUS, C.; TEK. TID.; 88, 211 (1958)

EATES, R. G.; BOWER, U. E.; CANHAM, R. G.; PRUE, J. E.; TRANS. FARADAY SOC.; 55, 2062 (1959)

BURDESE, A.; ABBADTISTA, F.; ATTI. ACCAD. SCI. TORINO, CL. SCI. FIS. MAT. NAT.; 93, 340 (1959)

DEWING, E. C.; RICHARDSON, F. D.; TRANS. FARADAY SOC.;

55,611 (1959)

FURUKAWA, G. T.; REILLY, M. L.; NATIONAL BUR STANDARDS; NES-IR 6484, CHAPT. 1 (1959)

KOEHLER, M. F.; COUGHLIN, J. P.; J. PHYS. CHEM.; 63, 605 (1959)

MATOO, B. N.; Z. PHYS. CHEM. (FRANKFURT AM MAIN); 19, 156 (1959)

MAURAS, H.; BULL. SOC. CHEM. FR.; 16 (1959)

NAIR, U. S. K.; NANCOLLAS, G. H.; J. CHEM. SOC.; (1959), 3934

ROEINSUN, R. A.; STOKES, R. H.; 'ELECTROLYTE SOLUTIONS' EUTTERWORTHS SCIENTIFIC PUBLICATIONS; 559 PP (LONDON, 1959) BEWLEY, D. K.; TRANS. FARADAY SOC.; 56, 1629 (1960)

BIANUCCI, G.; GHIRIHGHELLI, L.; ANN. CHIM. (ROME); 50, 99 (1960) 
GOGAR/THO GARRELS, R.M.; THOMPSON, M. E.; SIEVER, R.; AM. J. SCI $258,402(1960)$

GOJAN/LOR JANZ, G. J.; LORENZ, M.R.; U. S. DEFT. COMM. TECH. RFT [6], $156,833(1960)$

ŁOSEK/SET SEKIYA, M.; SETOYAMA, K.; KOGYO KAGAKU ZASSHI; 69, 1698 (1960)

GOWAR/ING WARNER, N. A.; INGRAHAM, T.R.; CAN. J. CHEM.; 38, 2196 11960;

61ELL/AND ELLIS, A. J.; ANDERSON, D. W.; J. CHEM. SOC. LONDON; 1765 (1961)

GIKEL/KIN KELLEY, K. K.; KING, E. G.; U.S. BUR. MINES; BULL 592 (1961)

EIMOR MORGAN, R. S.; J. CHEM. ENG. DATA; 6, 21 (1961)

GZAUS/NAI AUSTIN, J.M.; NAIR, A. D.; J. PHYS. CHEM.; 66, 519 (1962) EZBAK

62ELL/MOR BAKER, E. H.; J. CHEM. SOC.; (1962), 464

62HAL HALLA, F.; MUNATSH.; 93, 948 (1962)

EZJAK/TAN JAKUSZEWSKI, B.; TANIEWSKA-USINSKA, 5.; ROCZ. CHEM.; 36,329 (1962)

EZLIE,STO LIETZKE, M. H. STUUGHTON, R. W. J. PHYS. CHEM. 66, 508 tZMUR/GIA MURCH, L. E.; GIAUQUE, W. F.; J. PHYS. CHEM.; 6, 2002 (1962) EZRAT/MCC RATKOWSKY, D. A.; MCCARTHY, J.L.; J. PHYS. CHEM.; 66, 516 (1962)

62ZIE/SUL ZIELEN, A. J.; SULLIVAN, J. C.; J. PHYS. CHEM.; 66, 1065 (1962)

G3ADA/KEL ADAMI, L. H.; KELLEY, K. K.; U.S. BUR. MINES RPT. INUEST.; R.I 6260 (1963)

G3BAE/LIE BAECHMANN, K.; $67,802(1963)$

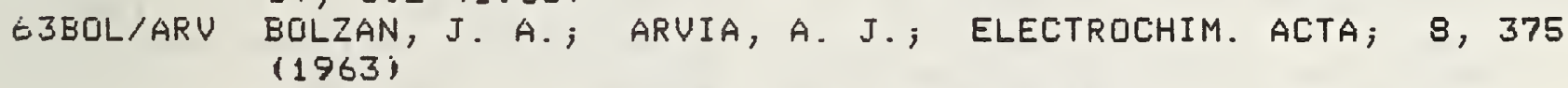
LIESER, K. H.; BER. BUNSENGES. PHYS. CHIM.;

63DAU/DAU DAUPHIN, J.; DAUPHIN, S.; CHATONIER, D.; ANDRAUD, G.; BULL. SOC. CHIM. FRANCE; 2754, (1963)

$63 E L L$ ELLIS, A. J.; AM. J. SCI.; 261, 259 (1963)

$63 \mathrm{HOS}$

E3HOSE

$63 J A N / K I R$ HOSTETLER, P. B.; J. PHYS. CHEM.; 67, 720 (1963) HUSTETLER, F. B.; AM. J. SCI.; 261, 238 (1963)

JANGG, G.; KIRCHMAYER, H.; Z. CHEM.; 3, 47 (1963)

E.JJOH/SUN JOHNSON, W. H.; SUNNER, 5.; ACTA. CHEM. SCAND.; 17,1917 (1963)

GJMEA/RIC MEADOWCRAFT, T. R.; RICHARDSON, F. D.; TRANS. FARADAY SOC.; $59,1564(1963)$

6.3RAB/HAR RABE, A.E.; HARRIS, J.F.; J. CHEM. ENG. DATA.; 8, 333 (1963)

$63 W I L$

$64 D E V / R U M$ $64 \mathrm{KOH} / Z A S$ G MMAR/SLU

6 $4 M C A$ WILLIX, R. L. S.; TRANS. FARADAY SOC.; 59, 1315 (1963)

DEVEZE, D.; RUMPF, P.; C.R. ACAD. SCI.; 258, 6135 (1964) KOHLER, K.; ZASKE, P.; Z. ANORG. ALLG. CHEM.; 331,1 (1964) MARSHALL, W. L.; SLUSHER, R.; JONES, E. V.; J. CHEM. ENG. DATA; 9, 187 (1964)

$64 M C A$ MCADIE, H. G.; CAN. J. CHEM.; 42, 792 (1964)

64NEW MCADIE, H. G.; MATER. RES. STD.; 4, 413 (1964) NEWMAN, E. S.; J. RES. NATL. BUR. STAND.; SEC. A; 68, 645 (1964) 
G $4 S I L / M A R$

SILLEN, L. G.; MARTELL, A. E.; 'STABILITY CONSTANTS

OF METAL-ION COMPLEXES', CHEM. SOC. LONDON, SPEC. PUBL.;

NO 17, 754 PP (LONDON, 1964)

65HOP/WUL HOPKINS, H. F.; WULFF, C. A.; J.PHYS. CHEM.; 69, 6 (1965) 65JAM/BRO JAMIESON, J. W. S.; BROWN, G. R.; GRUENER, D. W.; PEILUCK, R. U.; LAMONTAGNE, R. A.; CAN. J. CHEM.; 43, 2148 (1965)

65LAN

65MOH/RAY

E.SPAR

65SEI/LIN

LANGNUIRE, D. J.; J. GEOL.; 73,730 (1965)

MOHANTY, S. R.; RAY, M. N.; INDIAN J. CHEM.; 3, 37 (1965)

PARKER, $U$. B.; NATL. BUR. STANDARDS; NSRDS-NBS2 (1965)

SEIDELL, A.; LINKE, W. F.; 'SOLUBILITIES: INORGANIC AND

METAL-ORGANIC COMPOUNDS FOURTH EDITION'; VOL. I, D. UAN NOSTRAND CU, INC.; 1487 PP (PRINCETON, NEW JERSEY, 1958) AND

VOL. II, AMERICAN CHEMICAL SOCIETY; 1914 PP (WASHINGTON D. C., 1965)

6ESTE/WEI STERN, K. H.; WEISE, E. L.; NATL. BUR. STANDARDS;

NSRDS-NBS7, (1966)

67ATK/KOR

GSEUT

68HAL/TAS

GEHAM

G. BRAO/NAT

68SPE/WOO

69GAR/GLU

G9GAR/JEK

G9IZA/EAT

$675 I E / G I A$

TURAD

$71 \mathrm{JAN}$

$72 \mathrm{HAM} / W \mathrm{WU}$

$73 C 00$

7 AEDW

$74 J A C / L A N$

$74 \mathrm{PIT} / \mathrm{MAY}$

74THO/SMI

75KEA

75YOK/YAM

76GAR/PAR

76L IL/BR I

ATKINSON, G.; KOR, S. K.; J. PHYS. CHEM.; 71, 673 (1967)

BUTLER, J. N.; J. ELECTROANAL. CHEM. INTERFACIAL ELECTROCHEM.; $17,309(1968)$

HALLA, F.; VANTASSEL, R.; RADEX RUNDSCH; 1,27 (1968)

HAMER, W. J.; NATL. BUR. STANDARDS; NSRDS-NBS24, (1968)

RAO, G. U. S.; NATARAJAN, M.; RAO, C. N. R.; J. AM. CERAM. SOC.; $51,179(1968)$

SPEROS, D. M.; WOODHOUSE, R. L.; J. PHYS. CHEM.; 72, 2846 (1968)

GARDNER, A. W.; GLUECKAUF, E.; PROC. ROY. SOC. LONDON A313, 131 $(1969)$

GARDNER, W. L.; JEKEL, E. C.; COBBLE, J.W.; J. PHYS. CHEM.; 73, 2017 (1969)

IZAAT, R.M.; EATOUGH, D.; CHRISTENSEN, J.J.;

EARTHOLOMEW, C. H.; J. CHEM. SOC. A; (1969), 45

SIEMENO, P.R.; GRAUQUE, W. F.; J. PHYS. CHEM; 73, 149 (1969)

LOWELL, F. S.; 'A THEORETICAL DESCRIPTION OF THE LIMESTONE

INJECTION WET SCRUBBING PROCESS' RADIAN CORPORATION

FINAL REPORT; (CINCINNATI, OHIO, 1970)

'JANAF THERMOCHEMICAL TAELES' JANAF; NSRDS-NBS37, (1971)

HAMER, W. J.; WU, Y. C.; J. PHYS. CHEM. REF. DATA;

1,1047 (1972)

'CODATA TASK GROUP ON FUNDAMENTAL CONSTANTS', CODATA

EULLETIN, NO 11, DECEMBER, (1973)

EDWARDS, T. J.; THESIS, UNIV. OF CAL., BERKELEY; (1974)

JACOBSEN, R. L.; LANQMUIR, D.; GEOCHIM. COSMOCHIM. ACTA.; 38, $301(1974)$

FITZER, K. S.; MAYORGA, G.; J. SOLUTION CHEM.; 3, 539 (1974) THOMPSON, P.T.; SMITH, D.E.; WOOD, R. H.; J. CHEM. ENG. DATA; $19,386(1974)$

REARDON, E. J.; J. FHYS. CHEM.; 79, 422 (1975)

YUKOYAMA, H.; YAMATERA, H.; BULL. CHEM SOC. JAPAN; 48, 2708 (1875)

GARUIN, D.; PARKER, U. B.; WAGMAN, D. D.; EVANS, W. H.

'A COMBINED LEAST SUMS AND LEAST SQUARES APPROACH TO THE

EUALUATION OF THERMODYNAMIC DATA NETWORKS' NBSIR 76-1147, U. 5. DEPARTMENT OF COMMERCE, NATIONAL BUREAU OF STANDARDS, WASHINGTON, D. C., 20234, JULY, (1976)

LILLEY, T. H.; BRIGGS, C. C.; PROC. ROY. SOC. LONDON; A349, 355 (1976)

HUSS, A.; ECKERT, C. A.; J. PHYS. CHEM.; 81, 2269 (1977)

77HUS/ECK $77 P I T / R O Y$

FITZER. K. S.; ROY, R. N.; SILUESTER, L. F. J. AMER. CHEM. SOC.; 99, $4930(1977)$ 
$7750 L / V L A$ SOLOU'EVA, C. G.; VLASENKU, B. B.; KARAPETYANTS, M. KH. ZHUR. FIZ. KHIM.; 51, 2991, (1977)

$775 T A / N U T$ STAPLES, B. R.; NUTTALL, R. L.; J. FHYS. CHEM. REF. DATA; 6, $385(1977)$

78GOL/NUT GOLDBERG, R. N.; NUTTALL, R. L.; J. PHYS. CHEM. REF. DATA; 7, 263 (1978)

78STA

STAFLES, B.R.; ENUIRON. SCI. AND TECH.; 12, 339 (1978)

$79 F G B$

'WORKSHOP ON SULFUR CHEMISTRY IN FLUE GAS DESULFUR IZATION', U. S. DEPARTMENT OF ENERGY TECHNOLOGY CENTER, MORGANTOWN, W. VA., 26505, JUNE 7-8, (1979)

$80 I U P$ 'COMMISSION ON ATOMIC WEIGHTS OF IUPAC' PURE APPL. CHEM., $52, \quad 2349(1980)$

8OWAG/GAR WAGMAN, D. D.; GARUIN, D.; PARKER, U. B.; SCHUMM, R. H. PEDLEY, J. B.; 'NEW DEVELOPMENT IN THE EUALUATION OF THERMOCHEMICAL DATA', NATIONAL MEASUREMENT LABORATORY, 1979 TECHNICAL HIGHLIGHTS, NATIONAL BUREAU OF STANDARDS, NBS-SP572, U. S. GOUERNMENT PRINTING OFFICE, WASHINGTON, D. C., APRIL, (1980)

$81 \mathrm{GOL}$ GOLDBERG, R. N.; J. PHYS. CHEM. REF. DATA; ( IN PRESS)

$81 N B S$ WAGMAN, D. D.; EVANS, W. H.; PARKER, U. B.; HALOW, I.; BAILEY, S. M. ; SCHUMM, R. H.; NBS TECH. NOTE 270-3 (1968) IDEM, NBS TECH. NOTE 270-4 (1969) WAGMAN, D. D.; EVANE, W. H.; PARKER,U. B.; HALOW, I. H.; BAILEY, S. M.; SCHUMM, R. H.; CHURNEY, K. L.; NBS TECH. NOTE $270-5$ (19.71) PARKER, U. B.; WAGMAN, D. D.; EUANS, W. H.; NBS TECH. NOTE 270-6 (1971) SCHUMM, R. H.; WAGMAN, D. D.; BAILEY, S. M.; EUANS, W. H.; PARKER, U. B.; NBS TECH. NOTE 270-7 (1973) WAGMAN, D. D.; EUANS, W. H.; PARKER, U. B.; SCHUMM, R. H.; NUTTALL, R. L.; NBS TECH. NOTE 270-8 (IN PRESS)

$815 T A$ STAPLES, B. R.; J. PHYS. CHEM. REF. DATA ( IN PRESS ) 


\begin{tabular}{|c|l|l|l|}
\hline $\begin{array}{c}\text { U.S. DEPT. OF COMM. } \\
\text { BIBLIOGRAPHIC DATA } \\
\text { SHEET (See instructions) }\end{array}$ & $\begin{array}{l}\text { 1. PUBLICATION OR } \\
\text { REPORT NO. } \\
81-2345\end{array}$ & 2. Performing Organ. Report No. 3. Publication Date & September 1981 \\
\hline
\end{tabular}

4. TITLE AND SUBTITLE

A Report on Some Thermodynamic Data for Desulfurization Processes.

5. AUTHOR(S)

V. B. Parker; B. R. Staples; T. L. Jobe, Jr.; and D. B. Neumann

6. PERFORMING ORGANIZATION (If joint or other than NBS, see instructions)

NATIONAL BUREAU OF STANDARDS

DEPARTMENT OF COMMERCE

WASHINGTON, D.C. 20234

9. SPONSORING ORGANIZATIOH NAME AND COMPLETE ADDRESS (Street, CIty, StOte, ZIP)

7. ContracV/Grant No.

DoE A121-80-MC14004

8. Type of Report \& Period Covered

10. SUPPLEMENTARY NOTES

[ Document describes a computer program; SF-185, FIPS Software Summary, is attached.

11. ABSTRACT (A 200-word or less factual summary of most significant information. If document includes a significant bibliography or literature survey. mention it here)

Tables are presented here of values of thermochemical properties and processes at 298.15 $\mathrm{K}$ for substances of interest to DOE for flue gas desulfurization.

The substances covered are (1) the aqueous ions: $\mathrm{OH}^{-} ; \mathrm{SO}_{3}^{-2}, \mathrm{HSO}_{3}^{-}, \mathrm{SO}_{4}^{-4}, \mathrm{HSO}_{4}^{-}, \mathrm{CO}_{3}^{-2}$, $\mathrm{HCO}_{3}^{-}, \mathrm{H}^{+}, \mathrm{Mn}^{+2}, \mathrm{Fe}^{+2}, \mathrm{Mg}^{+2}, \mathrm{Ca}^{+2}, \mathrm{Na}^{+}$, and $\mathrm{K}^{+}$, and (2) solid, liquid, aqueous, and gaseous compounds or species formed from these ions.

The tables contain the following:

1. The thermochemical property values, enthalpy of formation, $\Delta_{f} H^{\circ}$, Gibbs energy of formation, $\Delta_{f} G^{\circ}$, entropy, $S^{\circ}$, and heat capacity, $C_{p}^{\circ}$ all at $298.15 k$, as well as the enthalpy difference between $298.15 \mathrm{~K}$ and $0 \mathrm{~K}, \mathrm{H}^{\circ}-\mathrm{H}^{\circ}$, for the basic species cited above

2. The predicted values for $\Delta H^{\circ}, \Delta G^{\circ}, \Delta S^{\circ}$, and $\Delta C_{p}^{\circ}$ as well as log $K$. (equilibrium constant) for the processes, or reactions, of importance to DOE, calculated from ( 1 ).

3 . The property values, $\phi_{L}$, the relative apparent molar enthalpy, $\gamma_{+}$, from the mean i.onic activity coefficient, and $\phi$, the osmotic coefficient, for binary aqueous systems at $298.15 \mathrm{~K}, \mathrm{a} 11$ as a function of concentration.

Some documentation for (2) and (3) is provided. All of the values given are consistent with the NBS TN 270 Series.

12. KEY WORDS (Six to twelve entries; alphabetical order; capitalize only proper names; and separate key words by semicolons) Activity coefficients; binary aqueous systems; enthalpy; enthalpies of dilution; entropy; flue gas desulfurizątion; Gibbs energy, osmoțic coffficjentş; thermochemical tables; $\mathrm{H}^{+} ; \mathrm{OH}^{-} ; \mathrm{S}$ ions; $\mathrm{CO}_{3}^{-2}$ and $\mathrm{HCO}_{3}^{-} ; \mathrm{Mn}^{-2} ; \mathrm{Fe}^{+2} ; \mathrm{Mg}^{+2} ; \mathrm{Ca}^{+2} ; \mathrm{Na}^{+} ; \mathrm{K}^{+}$

13. AVAILABILITY

$[X]$ Unlimited

For Official Distribution. Do Not Release to NTIS

Order From Superintendent of Documents, U.S. Government Printing Office, Washington, D.C. 20402.

14. NO. OF PRINTED PAGES

Order From National Technical Information Service (NTIS), Springfield, VA. 2216I 89

15. Price

$\$ 9.50$ 

